A typological investigation of personality:

Trait expression as a coordinated system of self-regulatory functioning

By

Laina M. Isler

\author{
A thesis \\ Submitted to Victoria University of Wellington \\ In fulfilment of the requirements for the degree of \\ Doctor of Philosophy \\ in Psychology
}

Victoria University of Wellington

2017 



\section{Statement of Authorship}

The three studies contained within this thesis have all been written for publication. I am the primary author on each paper, having developed the ideas, conducted and interpreted the analyses, and authored the final articles. Study 1 (Isler, Liu, Sibley, \& Fletcher, 2016) has been published by European Journal of Personality. Study 2 (Isler, Fletcher, Liu, \& Sibley, 2016) has been accepted for publication by Personality and Individual Differences, pending minor revisions. Study 3 is currently under review by European Journal of Personality. Both studies that have been accepted for publication are included in the present manuscript with permission from the respective journals.

Study 1 is the accepted version of the following article: Isler, L., Liu, J.H., Sibley, C.G., \& Fletcher, G.J.O. (2016). Self-regulation and personality profiles: Empirical development, longitudinal stability and predictive ability. European Journal of Personality, 30, 274-287, which has been published in final form at [http://onlinelibrary.wiley.com/doi/10.1002/ per.2054/epdf].

Study 2 is the accepted version of the following article: Isler, L., Fletcher, G.J.O., Liu, J.H, \& Sibley, C.G., (2017). Validation of the four-profile configuration of personality types within the Five-Factor Model. Personality and Individual Differences, 106, 257-262. 



\begin{abstract}
Many researchers contend that the intra-individual configuration of multiple traits, as compared to isolated trait dimensions, more effectively capture personality functioning at the level of the individual. Moreover, it is argued that by identifying subsets of individuals with similar trait-profiles, we can shed light on the generative mechanisms that underlie the expression and functioning of personality. Notably, self-regulatory systems of ego-resiliency and ego-control, as developed by Block and Block, comprise a valuable theoretical foundation for the development and interpretation of personality types. However, much of the literature on this topic is theoretically ambiguous, and is afflicted with inconsistent empirical outcomes across studies. The focus of the present thesis is to advance the empirical assessment and theoretical interpretability of personality profiles. More particularly, comparisons between three and four profile solutions are at the core of the current investigations. These competing models reflect differing interpretations of egodomains; the three profile model embodies a proposed curvilinear relationship between the two constructs, whereas the four profile model emphasises the roles of ego-resiliency and ego-control as distinct mechanisms with unique outcomes.
\end{abstract}

In Study 1, Latent Profile and Latent Transition Analyses were conducted using the SixFactor Model of personality. Three and four profile solutions were developed and compared using measures of model fit, profile interpretability, longitudinal stability, and predictive ability. In Study 2, the replicability of both solutions when using the Five-Factor Model was examined, along with the comparative value of the Six- as opposed to FiveFactor Model in profile identification. Finally, in Study 3, the interpretability of the four profile solution as reflecting differentiated domains of ego-functioning was assessed using established measures of ego-resiliency and ego-control. Across all three studies, profiles were considered using a novel approach utilising both continuous and categorical methodologies. Rather than treating profiles as entirely discrete groupings of personality functioning, the present investigation considers convergence with prototypical profiles in terms of degree.

The current findings provide support for the four profile solution as a more coherent and theoretically validated model as compared to the three profile solution. Specifically, although both solutions demonstrated good fit and longitudinal stability, the four profile 
solution was associated with more theoretically interpretable outcomes. These findings were consistent when using both the Six- as well as Five-Factor Model of personality; however, omission of the sixth trait resulted in a reduction in profile precision and explanatory power. Finally, in Study 3, selected exemplars of the four profile solution converged onto theoretically consistent domains of high/low ego-resiliency and ego-control. Block and Block's model of ego-resiliency and ego-control provides a clear unifying framework for the intra-individual structuring of a four profile configuration of traits. Egoconstructs are flexible self-regulatory mechanisms that develop through reciprocal personenvironmental transactions. The current results are therefore suggestive of a hierarchical relationship between ego-domains and personality traits, whereby traits form the basic parameters of a dynamic self-regulatory system. Moreover, the combination of continuous and categorical methodologies presently used strengthens the conclusions and arguments in this thesis. 


\section{Acknowledgements}

The development and completion of a PhD thesis is a challenging yet thoroughly rewarding experience. I am immensely grateful, and humbled, by the extraordinary guidance and support which has seen me through this process.

I would like to thank my supervisors Prof James Liu and Prof Garth Fletcher. James took me on as a (very) green student, and enthusiastically provided me with a wealth of opportunities to expand my horizons. Through Garth's guidance and encouragement, I was able to sharpen my scientific thinking and develop the skills necessary to create a thesis of which I am truly proud. I am extremely grateful for having had the opportunity to learn from such a meticulous and supportive researcher. I am also indebted to Chris Sibley, who graciously permitted me the use of his longitudinal dataset, the New Zealand Attitudes and Values Survey. The analyses I was able to perform stand on the quality of this dataset.

I would also like to acknowledge the friendships I have made here at Victoria University. Getting to know and work with such a fantastic group of people has been an invaluable experience, and never fails to brighten my day. Particularly, I would like to thank Ágnes Szabó, whose poise and skill in completing her own thesis is an inspiration, and Lorance Taylor, whose light-hearted banter was always a welcome distraction. I would also like to thank Tamara Qumseya, Reneeta Morgan and Sam Pobog-Jaworowski, who saw me through my final milestones and encouraged me to stay positive, clear headed, and determined.

I am especially grateful to my beautiful family. My parents, who encouraged me to cast a wide net of experiences, and to pursue all of my interests with vigour. My three brilliant siblings, who have all pursued their own goals with drive and determination and are all role models for what can be achieved through creativity and persistence.

Most of all, I want to thank my partner Philip Connor, who believed that I could do this before the thought had crossed my mind, and then worked with me every step of the way to see it through (from application to hand-in). Phil, you have contributed to every part of this process. You have been my sounding board for every idea, rethink, revision, and rewrite, and supported me through every disappointment and victory. You have thought always of my well-being, made me countless dinners and packed lunches, and encouraged me to insert breaks and relaxation into my busy schedule (often to my great annoyance). Phil, there is honestly no way to convey how integral you have been to this entire process.

Thank you so very much. 



\section{Table of Contents}

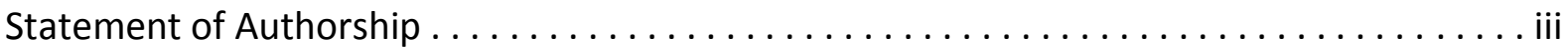

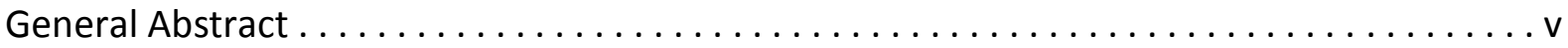

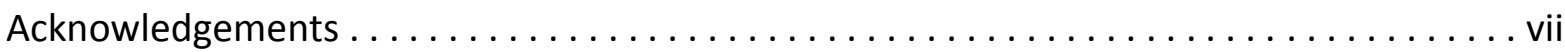

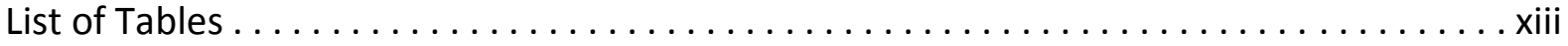

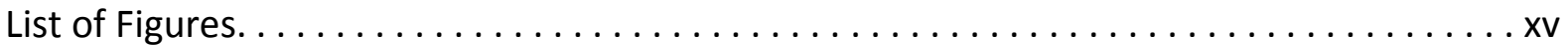

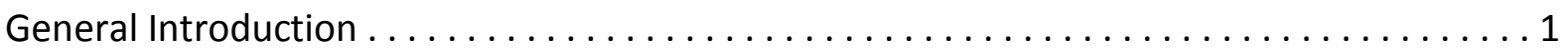

Brief History of the Modern Individual Difference or Trait Approach . . . . . . . . . . 2

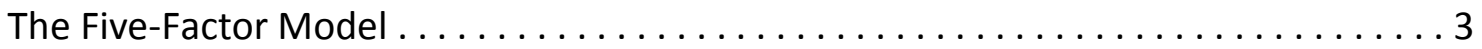

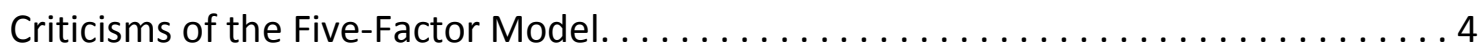

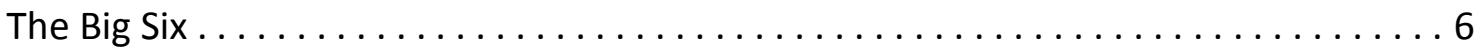

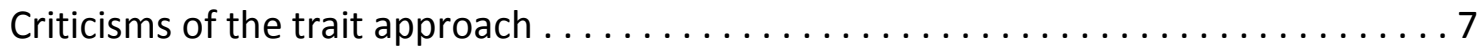

Types and Traits as Complementary Mechanisms $\ldots \ldots \ldots \ldots \ldots \ldots \ldots \ldots$

Ego-constructs and personality typologies $\ldots \ldots \ldots \ldots \ldots \ldots \ldots \ldots \ldots$

Controversies and complexities in type development $\ldots \ldots \ldots \ldots \ldots \ldots \ldots \ldots$

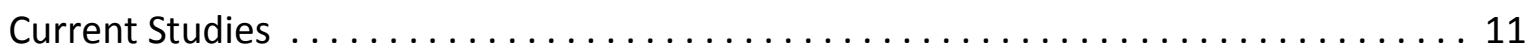

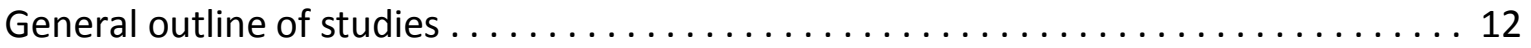

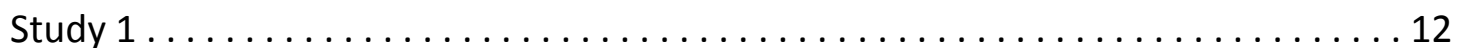

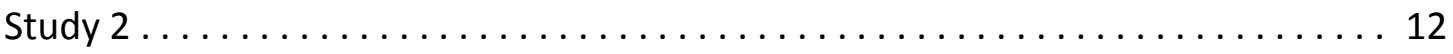

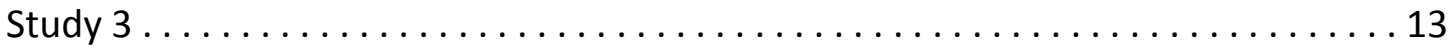

STUDY 1: Self-Regulation and Personality Profiles: Empirical Development, Longitudinal Stability, and Predictive Ability . . . . . . . . . . . . . . 15

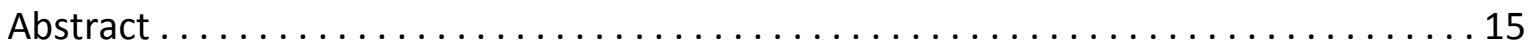

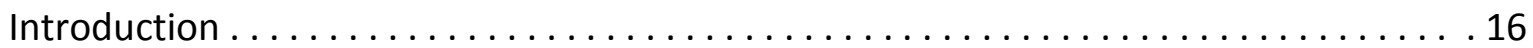

Self-Regulation within an Interpersonal Context $\ldots \ldots \ldots \ldots \ldots \ldots \ldots \ldots \ldots \ldots$

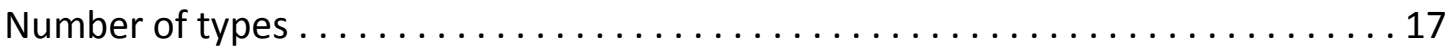

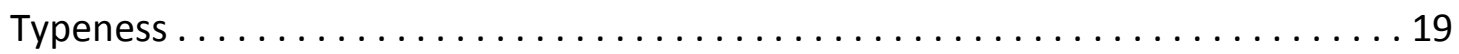

Predictive validity of personality profiles $\ldots \ldots \ldots \ldots \ldots \ldots \ldots \ldots \ldots \ldots \ldots \ldots \ldots \ldots \ldots$

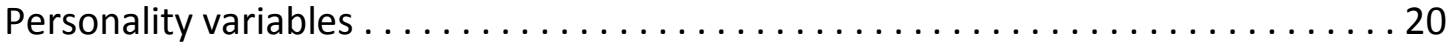

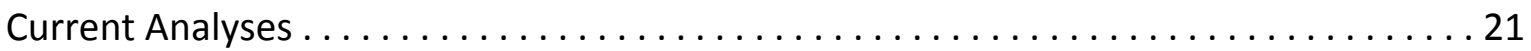

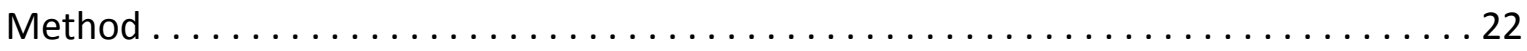




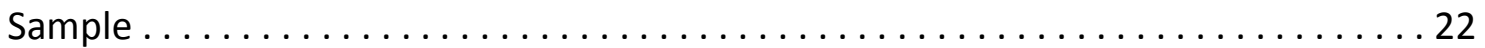

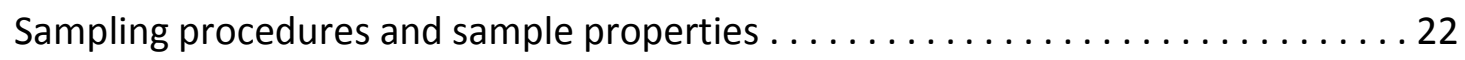

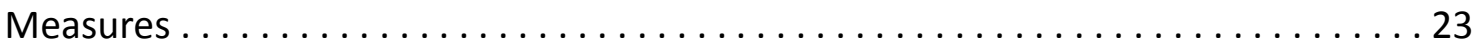

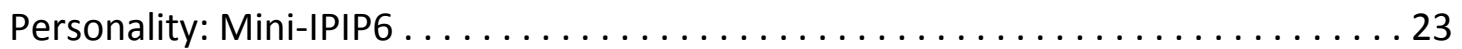

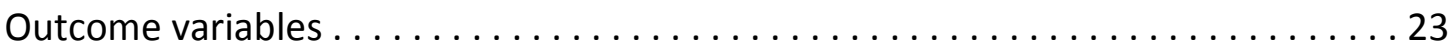

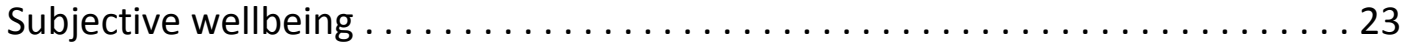

Perceived quality of interethnic relations $\ldots \ldots \ldots \ldots \ldots \ldots \ldots \ldots \ldots \ldots \ldots$

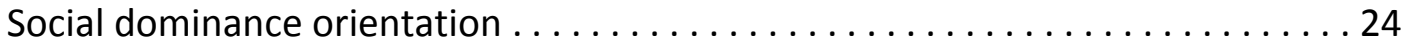

Self-enhancement and openness to change values $\ldots \ldots \ldots \ldots \ldots \ldots 24$

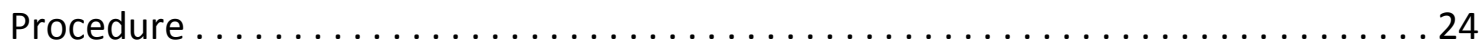

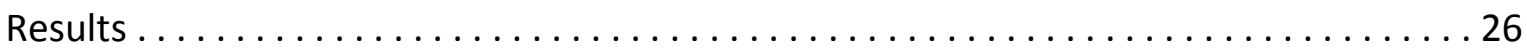

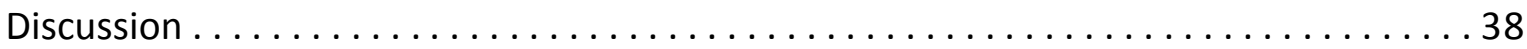

Fit and Stability of Three and Four Profile Solutions $\ldots \ldots \ldots \ldots \ldots \ldots \ldots \ldots$

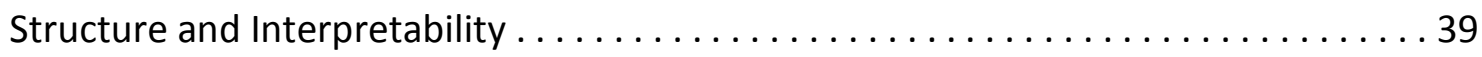

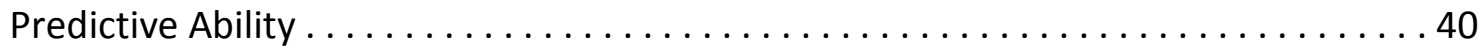

Comparisons across Three and Four Profile Solutions $\ldots \ldots \ldots \ldots \ldots \ldots \ldots \ldots$

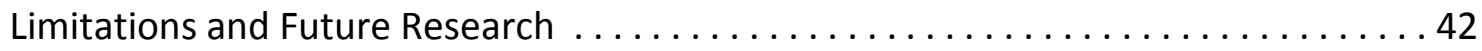

STUDY 2: Validation of the Four Profile Configuration of Personality Types within the

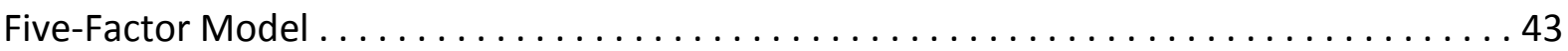

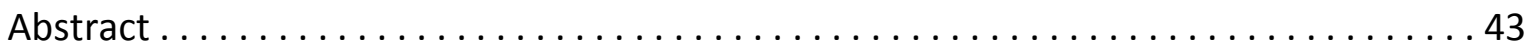

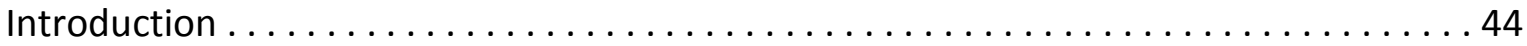

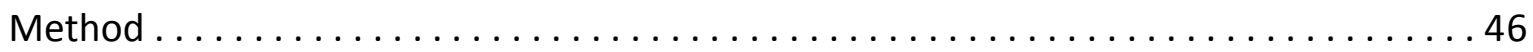

Sample ................................. 46

Measures ................................... 46

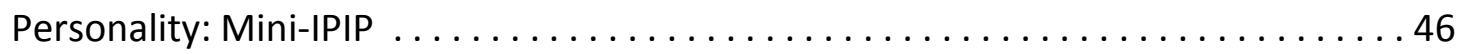

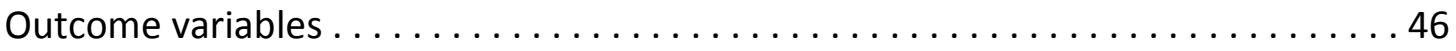

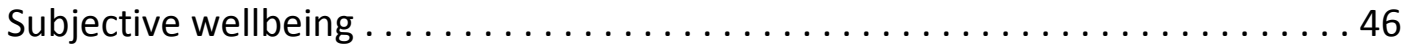

Perceived quality of interethnic relations $\ldots \ldots \ldots \ldots \ldots \ldots \ldots \ldots \ldots \ldots \ldots \ldots \ldots$

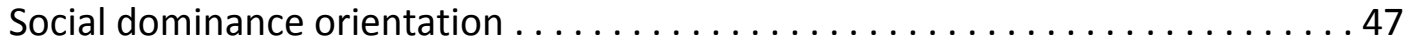

Self-enhancement and openness to change values $\ldots \ldots \ldots \ldots \ldots \ldots \ldots 7$

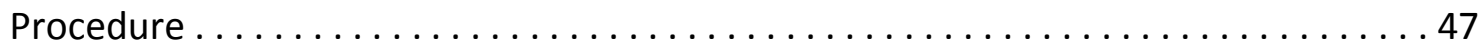

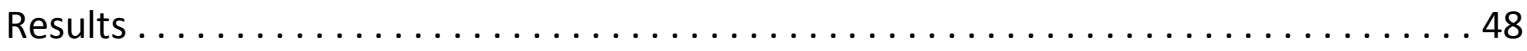




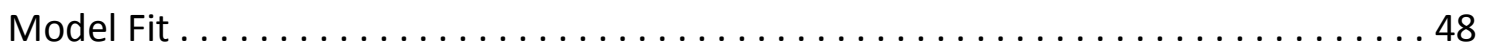

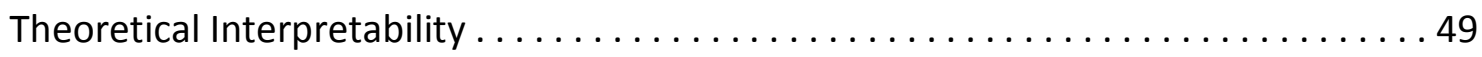

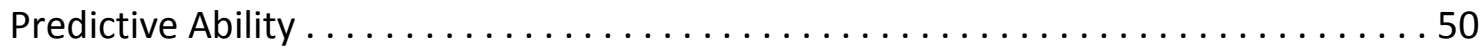

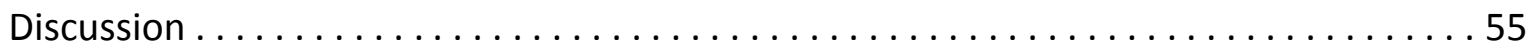

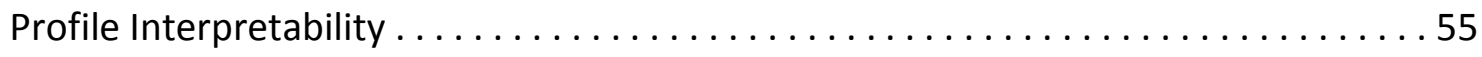

Limitations, Future Research, and Conclusions .................. 56

STUDY 3: Validation of the Four Profile Model of Personality Types: Empirical Convergence

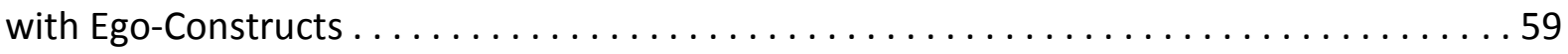

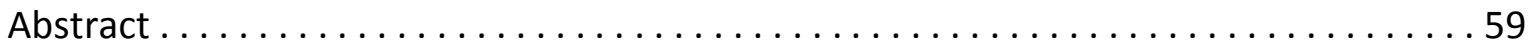

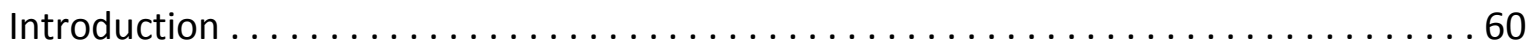

Relationships between Ego-Constructs $\ldots \ldots \ldots \ldots \ldots \ldots \ldots \ldots \ldots \ldots \ldots \ldots \ldots \ldots \ldots \ldots \ldots$

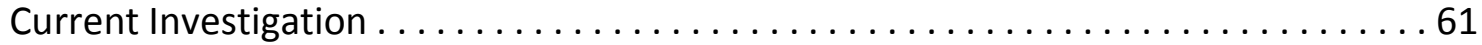

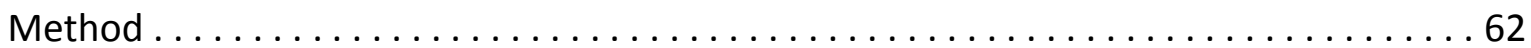

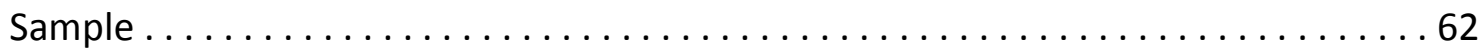

Sampling procedures and composition $\ldots \ldots \ldots \ldots \ldots \ldots \ldots \ldots \ldots \ldots \ldots \ldots \ldots \ldots$

Measures ........................................64

Ego-resiliency and ego-control . ...........................6 64

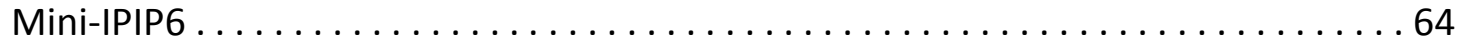

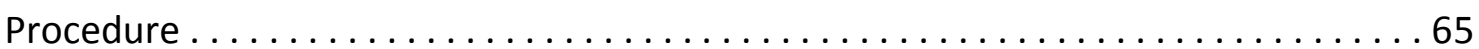

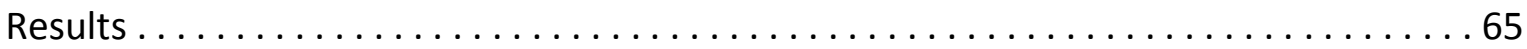

Four Profile Solution: Trait Configurations and Model Fit $\ldots \ldots \ldots \ldots \ldots \ldots \ldots$

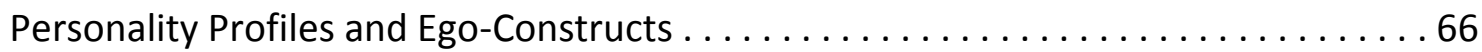

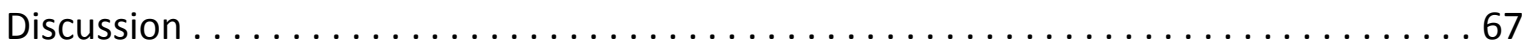

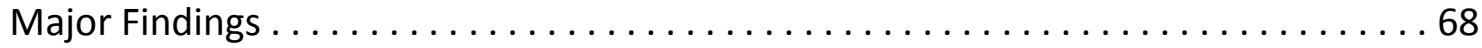

Implications, Limitations, and Future Directions $\ldots \ldots \ldots \ldots \ldots \ldots \ldots \ldots \ldots \ldots \ldots \ldots$

General Discussion .................................. 71

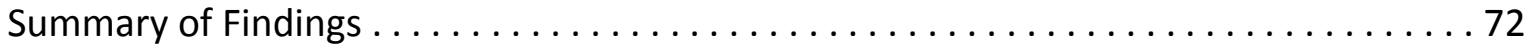

Complementary Functioning of Types and Traits $\ldots \ldots \ldots \ldots \ldots \ldots \ldots \ldots \ldots \ldots \ldots \ldots \ldots$

Personality Types within Context . . . . . . . . . . . . . . . . . . . . 74

Typeness ....................................... 76

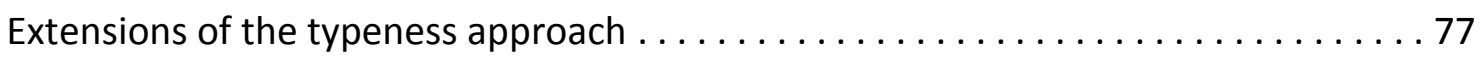

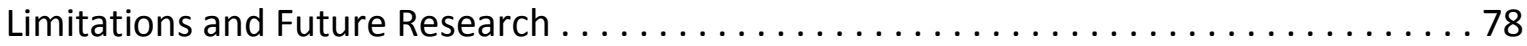




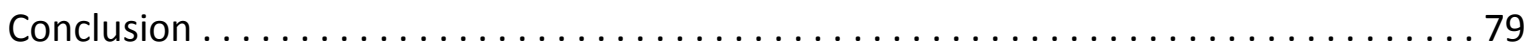

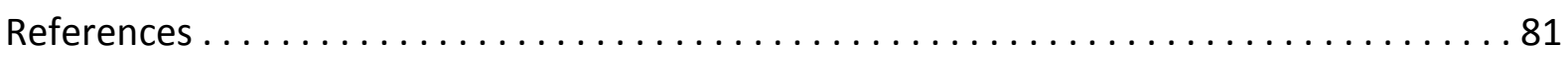

Appendix A: Personality Scale Factor Loadings and Descriptive Statistics . . . . . . . . . . 94

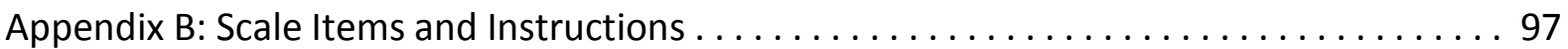




\section{List of Tables}

Table 1. Costa and McCrae's (1995) Five-Factor Model: Domains and facets . . . . . . . . . 4

Table 2. Six-Factor Model personality profiles: Fit statistics for 3 and 4 profile solutions . . . 28

Table 3. Participant distribution in SFM personality profiles: Percent of total sample ... . . 30

Table 4. Participant distribution in SFM personality profiles: Percent of profile overlap . . . 30

Table 5. SFM personality profile membership regressed on demographic variables: Standardised beta weights . . . . . . . . . . . . . . . . . . . . . 31

Table 6. Beta weight of SFM profile membership regressed on outcome variables. .

Table 7a. SFM four profile solution: One-way ANOVAs predicting mean-level differences in outcome variables

Table 7b. SFM three profile solution: One-way ANOVAs predicting mean-level differences in outcome variables

Table 8. Sex, age, and income among low, medium and high attrition rates: Tuckey Post Hoc analyses ................................. 35

Table 9. Transition probabilities of SFM profiles across 1-year and 2-year intervals . . . . . 36

Table 10. Nested model: BIC difference between freely estimated and constrained models .37

Table 11. Five-Factor Model (FFM) personality profiles: Fit statistics for 3 and 4 profile

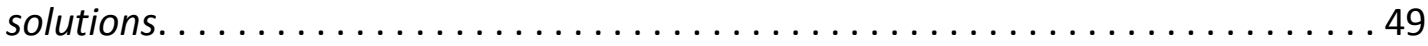

Table 12. Participant distribution in FFM personality profiles: Percent of total sample . . . 49

Table 13. FFM personality profile membership regressed on demographic variables: Standardised beta weights.

Table 14. Beta weight of FFM profile membership regressed on outcome variables. .

Table 15. FFM four profile solution: One-way ANOVAs predicting mean-level differences in outcome variables 
Table 16. FFM three profile solution: One-way ANOVAs predicting mean-level differences in outcome variables . . . . . . . . . . . . . . . . . . . . 54

Table 17. Prototypical respondents: Response rate and average probability of class

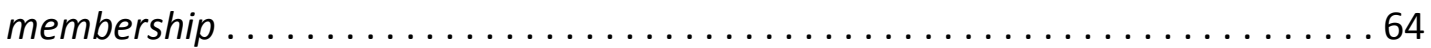

Table 18. Means and standard deviations of ego-constructs as a function of personality

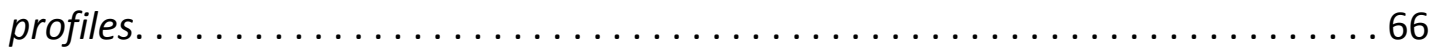




\section{List of Figures}

Figure 1a. Three Profile solution of personality traits (Six-Factor Model) . . . . . . . . . . . 29

Figure 1b. Four Profile solution of personality traits (Six-Factor Model) . . . . . . . . . . 29

Figure 2a. Four Profile solution of personality traits (Five-Factor Model) . . . . . . . . 50

Figure $2 \mathrm{~b}$. Three Profile solution of personality traits (Five-Factor Model) . . . . . . . . 50

Figure 3. Proposed relationship between ego-constructs and personality profiles ....... 62

Figure 4. Four profile solution among prototypical respondents . . . . . . . . . . . . . . 66 



\section{General Introduction}

Individual differences in thoughts, feelings, and behaviour are at the core of personality psychology. Consequently, particular variables are typically used by personality researchers as the primary units of measurement to identify and describe differences across individuals. In contrast to this variable-centred approach, many researchers contend that the withinperson configuration of traits represents a critical aspect of human individuality. As Donnellan and Robins (2010) argue, whole individuals, rather than isolated traits, experience and engage with their environments. Accordingly, dynamic transactions between individuals and the social environment may be more effectively understood from a person-centered (or typological) perspective.

The appeal of a person-centred approach to personality research is readily apparent. This methodology emphasises a focus on the intraindividual structure of personality, and encourages researchers to consider the constellation of personality characteristics at an individual level. A useful analogy is to consider how we describe, interpret, and understand illnesses (Robins \& Tracy, 2003). Much information regarding developmental trajectories and outcomes relating to illness would be lost if we considered each symptom (congestion, cough, fever) in isolation. By considering the co-occurrence of these symptoms, we can describe and address the illness as a coherent entity.

Profiles or structures that group individuals also provide greater conceptual clarity than trait models. Through characterising groups of individuals by common constellations of traits, we can efficiently collapse across a broad range of descriptors into a concise typology. This latter approach, I shall argue, gives insight into the underlying etiology driving the relationship among the traits, thus delivering a richer and more coherent theory of personality.

Despite their apparent usefulness, however, traditional investigations into personality typologies have seldom held up to rigorous empirical standards of replicability, construct validity, and generalizability. Particularly, a common criticism of early type-theorists is of their over-reliance on 'armchair theoretical speculation' (Robins \& Tracy, 2003). To avoid this fate, contemporary theorists rely on more rigorous statistical techniques and evidence to develop and interpret personality structures. Care also must be taken in choosing the trait dimensions on which the personality patterns are based, as well as the population and samples used, as these decisions will influence the resultant profiles. 
The current set of studies in this thesis contributes to the understanding of typological structures of personality using novel statistical techniques. This introduction provides a brief overview of the contemporary status of typological research as a foundation for the three subsequent investigations, either submitted for publication or already published. I will first consider the historical development of a coherent taxonomy of personality traits, and the emergence of the commonly adopted Five-Factor Model, along with associated issues and controversies. I will then describe and provide evidence for an alternative Six-Factor Model of personality. The benefits and limitations of trait approaches more generally will be addressed, and the usefulness of type-based approaches as a complement to the traditional trait-based approach will be advanced.

Following this, Block and Block's (1980) theory of ego-functioning will be described as a theoretical framework for organizing and interpreting configurations of personality. Specific investigations using this model as a basis for typological research will be reviewed. I will then consider some controversies and complexities (both methodological and theoretical) concerning the development and interpretation of personality typologies. Finally, I will briefly review the three studies in the thesis.

\section{Brief History of the Modern Individual Difference or Trait Approach}

The modern classification of personality into a coherent taxonomy emerged from several decades of rigorous research and validation using factor analysis to sort individual characteristics into relatively independent groups of related traits. The advantage of this approach is that it classifies a large spectrum of traits into a small coherent number of dimensions. However, due to competing ideas about which variables should be included in the analysis, early researchers disagreed about the number of personality factors in the final model (for reviews, see Digman, 1990; McCrae \& Costa, 2008).

The lexical approach to personality investigations was devised to provide a solution to this problem. This paradigm assumes that traits with the most importance and meaning are encoded within language. Trait structures could then be assessed by statistically analysing clusters and relationships between common trait terms, thus revealing the underlying structure of personality. Using this framework, Cattell (1943) developed 35 clusters of traits from common adjectives describing personality characteristics. However, Cattell's taxonomy of traits did not hold up to numerous attempts at replication (Eysenck \& Eysenck, 1969; 
Howarth \& Brown, 1971; Sell, Demaree, \& Will, 1970), and was quickly criticised for being too complex (Fiske, 1949).

Early evidence for a more parsimonious model of personality was advanced by Tupes and Christal (1961), who factor analysed Cattell's dimensions, and found evidence for five higher-order factors (Surgency, Agreeableness, Dependability, Emotional Stability, and Culture), which bear striking similarities to the modern Five-Factor Model. Although this solution was later replicated (Norman, 1963), Tupes and Christal's contributions, and the five-factor model of personality, went largely unnoticed for two decades. It was not until the early 1980s that this model was revisited and replicated by Goldberg (1981), who applied factor analytic strategies to consider exhaustive lists of traits used in the English language.

Despite the elegance of these lexically-derived solutions, lexical studies into personality failed to gain initial traction as competing models of personality were considered more scientific than using lay terms as the empirical base. However, the Five-Factor Model of personality came to dominate the field of personality research when studies demonstrated that the various traits assessed by prominent personality questionnaires were closely related to the lexical Big Five (Costa \& McCrae, 1988; McCrae, 1989; McCrae \& Costa, 1985; McCrae \& Costa, 1989). Consequently, the Five-Factor Model of personality has become the most widely accepted trait-model of personality.

The Five-Factor Model. The Five-Factor Model organises personality around five overarching trait-domains which explain the common variance among facets and lowerorder traits. More particularly, facets are specific and unique aspects that are together reflective of the cognitive, affective, and behavioural tendencies comprising broader personality domains (for instance, Self-Discipline and Orderliness are two facets of Conscientiousness). Likewise, facets are identified through the combination of multiple covarying lower-order traits, which make up the basic unit of analysis (for instance, items ' am always prepared' and 'I carry out my plans' combine to reflect an individual's level of Self-Discipline). Although there has traditionally been mixed agreement on the specification of lower-level traits (see Costa \& McCrae, 1995; McCrae \& Costa, 2008), the five domains reflected in the Five-Factor Model can be readily summarised: Extraversion (versus introversion), Agreeableness (versus antagonism), Conscientiousness (versus indirectedness), Neuroticism (versus emotional stability), and Openness to Experience 
(versus closedness). Costa and McCrae (1995) further differentiated each broad domain into six more specific facets based on a review of the literature, and item analyses (facet solution is briefly summarised in Table 1).

Table 1

Costa and McCrae's (1995) Five-Factor Model:

Domains and facets

\begin{tabular}{lllll}
\hline Neuroticism & Extraversion & Openness & Agreeableness & Conscientiousness \\
\hline Anxiety & Warmth & Fantasy & Trust & Competence \\
Angry Hostility & Gregariousness & Aesthetics & Straightforwardness & Order \\
Depression & Assertiveness & Feelings & Altruism & Dutifulness \\
Vulnerability & Activity & Actions & Compliance & Achievement Striving \\
Impulsiveness & Excitement-Seeking & Ideas & Modesty & Self-Discipline \\
Self-Consciousness & Positive Emotions & Values & Tender-Mindedness & Deliberation \\
\hline
\end{tabular}

The utility of the Five-Factor Model of personality is evidenced in the scope of investigations demonstrating real-life consequences of personality measures in personal, interpersonal, and social domains, including happiness and wellbeing, peer acceptance and friendship, physical health and psychopathology, occupational attainment, criminality, as well as political attitudes and values (for a detailed review, see Ozer \& Benet-Martínez, 2006; Roberts, Kuncel, Shiner, Caspi, \& Goldberg, 2007). Moreover, longitudinal investigations suggest that personality traits are remarkably consistent over time (Fraley \& Roberts, 2004; Roberts \& DelVecchio, 2000), while patterns of change in traits occurring over the life course are eminently interpretable (see Roberts, Walton, \& Viechtbauer, 2006). Finally, notwithstanding some conflicting reports (De Raad et al., 2010; Saucier \& Goldberg, 2001), growing evidence suggests that a reasonable approximation of each of the five domains reflected in the Five-Factor Model generalises across cultures (McCrae \& Allik, 2002; McCrae \& Costa, 1997; McCrae et al., 2005; Piedmont \& Chae, 1997).

Criticisms of the Five-Factor Model. A major advantage of the Five-Factor Model of personality lies in its ability to provide a common framework for psychological research. However, disagreements surrounding the appropriate facet-structure of overarching domains have resulted in difficulties in clearly defining and describing the factors. For instance, Costa and McCrae (1995) subsume warmth as a facet of Extraversion, whereas 
Goldberg (1981) views this as reflecting Agreeableness. Impulsivity is typically considered by Costa and McCrae (1995) as reflecting Neuroticism but this facet has likewise been subsumed under Conscientiousness (1992). As a result of these complexities, there is currently no consensus regarding the identification and coding of facets (see McCrae \& Costa, 2008).

In addition to issues surrounding facet identification, the classification of major trait domains remains a fundamental problem in personality psychology. Block (1995) expressed concern that the development of the NEO-PI-R instrument was overly concerned with reflecting the lexical Big Five dimensions, rather than relying on more data-driven techniques. Indeed, the tendency to rely on the assumptions of the Big Five as a 'universal descriptive framework... for the comprehensive assessment of individuals' (McCrae, 1989, p. 243) may reduce the likelihood of identifying other potentially valid and reliable domains of personality.

This concern is reflected in a number of investigations capturing additional variance not represented by the traditional five-dimensional models. For example, estimations of a fivefactor model using psycholexical techniques in Hungary (De Raad \& Szirmak, 1994; Szirmak \& De Raad, 1994) and Italy (Di Blas \& Forzi, 1998, 1999) resulted in four factors plausibly reflecting the Big Five Agreeableness, Neuroticism, Extraversion and Conscientious, with a fifth factor reflecting tendencies towards trustworthiness versus greed and conceitedness. Only by extracting and rotating six-factors were researchers able to obtain an Intellect(Openness)-related factor, thereby completing the Big Five (plus one).

Ashton et al. (2004) argues that the identification of an additional Integrity/Trustworthiness domain (in the research just described) suggests that solutions converging on the traditional five-factor structure may likewise overlook a similar trustworthiness variable. Indeed, this six-factor structure has been repeatedly demonstrated in several psycholexical studies of personality, including Dutch (De Raad, 1992), Korean (Hahn, Lee, \& Ashton, 1999), and French (Boies, Lee, Ashton, Pascal, \& Nicol, 2001) samples. Comparisons of independent psycholexical studies of personality across a wide-variety of languages and cultures have likewise provided support for the generalizability of the Six-Factor Model (Ashton et al., 2004; Lee \& Ashton, 2008; Saucier, 2009). 
The Big Six. Based on the growing support for a replicable and interpretable sixth factor, Lee and Ashton (2004) posited a new structural model of personality (known as HEXACO). This new framework identifies six domains of personality: Honesty-humility $(H)$, Emotionality (E), eXtraversion (X), Agreeableness (A), Conscientiousness (C), and Openness to Experience (O). Briefly summarised, HEXACO dimensions reflecting Extraversion, Conscientiousness, and Openness to Experience correspond closely to their Five-Factor Model counterparts. The remaining three variables identified within the HEXACO model incorporate variance associated with Big Five Agreeableness and Neuroticism, as well as additional variance not captured by the Big Five. More specifically, sentimentality traits associated with the Big Five Agreeableness are incorporated in the HEXACO model under Emotionality, whereas anger-related traits associated with the Big Five Neuroticism are incorporated in the HEXACO framework under Agreeableness (at its low pole). Finally, Honesty-humility incorporates and extends facet information relating to Straightforwardness and Modesty, which are traditionally integrated into the Big Five Agreeableness domain.

Proponents of the Six-Factor Model contend that the six factors reflected in the HEXACO framework more effectively capture personality variance than the traditional FiveFactor Model (Ashton \& Lee, 2008). In contrast, some researchers have suggested that the conceptual and empirical overlap between Honesty-humility and the Big Five Agreeableness domain negates the need for an alternative HEXACO scale (McCrae \& Costa, 2008; Van Kampen, 2012). However, comparisons of the explained variance across the two models demonstrates that the five domains reflected in the Five-Factor Model cannot fully capture the variance of the six-dimensional HEXACO space (Gaughan, Miller, \& Lynam, 2012). The incremental utility of this additional factor has been further shown in numerous studies. For instance, the HEXACO model of personality outperformed the Five-Factor Model in predicting outcomes relating to romantic relationship strategies and power (Lee et al., 2013), egoism (De Vries, De Vries, De Hoogh, \& Feij, 2009), as well as materialism, social adroitness, delinquency, and unethical decision making (Ashton \& Lee, 2008).

A further critique of the HEXACO model suggests that Honesty-humility merely elevates facet-level aspects of Agreeableness to a domain level of analysis. As facet-level information can provide incremental utility beyond the broader trait domains (Ashton, Paunonen, \& Lee, 2014; Paunonen \& Ashton, 2001), the argument could be advanced that the incremental 
utility of Honesty-humility reflects a measurement artefact. However, as noted by Ashton et al. (2014), identification of the sixth factor was not achieved by artificially targeting and elevating facet information, but instead reflected coherent and consistent outcomes of repeated lexical testing across a broad range of cultures and languages. These consistent results support the importance of treating Honesty-humility as a separate factor, rather than as a spurious measurement artefact.

Criticisms of the trait approach. The variable(trait)-centred methodology discussed above reflects an effort to organise and categorise the between-person variation manifested within personality. Notably, trait investigations of personality effectively identify and explain real-life outcomes and individual differences regardless of whether the traditional five-factor or the alternative six-factor approach is used.

However, trait-based approaches are associated with a number of limitations. Briefly summarised, criticisms of the variable-centred methodology are organised around the inability of trait-models to explain (rather than describe) personality processes, account for the whole person, or address individual-level dynamic and developmental processes. Particularly, the development of the Five-Factor Model is accused of being 'atheoretical' (Block, 1995; Block, 2010), and is acknowledged by its proponents as providing a descriptive structure of traits based on empirical observation, rather than a theoretical or explanatory context for interpreting how traits function in daily life (McCrae \& Costa, 2008). Accordingly, the underlying mechanisms that produce variation within the observed dimensions remain obscure.

Compounding this issue, the trait-approach treats personality domains as orthogonal, implying that the expression of each dimension operates independently within each individual. This approach seems implausible given that personality traits almost certainly comprise a dynamic system within each individual. Instead, it is eminently likely that the complex interplay among personality dimensions provides a more accurate description of how personality is structured at an individual level (Block, 2010). For instance, an individual with low levels of Extraversion would be likely to experience and express that trait differently depending on their relative levels of Emotional Stability and Agreeableness. Thus, although trait domains comprehensively summarise individual differences between persons, 
they fail to account for within-person causal dynamics (Caprara \& Cervone, 2000; Lamiell, 1997).

These criticisms highlight the need for a model of personality reflecting higher-order dispositional constructs, rather than purely classificatory structures (Block, 2010; Harré, 1998). Beyond focusing on the between-person differences on isolated personality characteristics, such an approach should provide a theoretical foundation to organise, explain, and interpret personality at the level of the individual.

\section{Types and Traits as Complementary Mechanisms}

Within- and between-person theorizing and methodologies are commonly interpreted as operating in opposition. However, these two approaches may be quite compatible. Indeed, by identifying groups or subsets of individuals with similar configurations of traits (referred to as types, or profiles), researchers may be able to provide greater insight into the underlying mechanisms that produce both within-person variation and between-person differences across the observed dimensions. Thus, a type-based perspective may function as a complement rather than a substitute to the variable-centred approach (Donnellan \& Robins, 2010).

A theory often advanced as providing a framework for understanding and interpreting a type-based configuration of personality traits is Block and Block's (1980) model of egocontrol and ego-resiliency (Asendorpf, Borkenau, Ostendorf, \& van Aken, 2001; Gramzow, Sedikides, \& Panter, 2004; John, 1990). Briefly summarised, Block and Block postulated that patterns of human experience and functioning emerge from a dynamic process whereby the individual, inclusive of genetic and temperamental factors, experiences and reacts to environmental contingencies. For instance, a child with high levels of impulsivity will receive feedback regarding the extent to which an environment is supportive and stable, or disordered and permissive. The child will then incorporate this feedback, negotiate the links between their internal motivations and external opportunities and constraints, and develop a working model for perceiving and reacting to the social environment. Through repeated experiences, these general models form an affective and cognitive framework, which motivate and guide an ongoing flux of affect, cognition, and behaviour.

The complementary nature of ego-constructs and trait domains is readily apparent when one considers the roles of these dimensions. As I will later address in more detail, 
Block and Block's model of personality accounts for dynamic self-regulatory processes, which in turn generate individual differences in characteristics and behaviours. In comparison, trait-models of personality provide descriptive interpretations, and account more narrowly for the primary dimensions embodied in individual differences (John, 1990). Thus, trait-models and ego-constructs operate on different levels of description, and reflect hierarchically related aspects of personality functioning. On this account, traits can be understood as the parameters of an adaptive system, and the specific patterns or configurations of traits within the individual reflect and sustain higher-order ego-constructs.

Ego-constructs and personality typologies. Block and Block (1980) characterised egocontrol in terms of the degree to which an individual will express or contain impulses and affectively driven motivations. Both high and low levels of ego-control have the potential to be associated with both positive and negative outcomes: High levels can facilitate disciplined and directed behaviour, along with a general discomfort with ambiguity and change; low levels can promote a curiosity and a desire to explore, along with distractibility and restlessness. Alternatively, ego-resiliency is conceptualised as an adaptive capacity to respond flexibly to the dynamic requirements of changing circumstances. Unlike egocontrol, ego-resiliency is understood as varying from desirable to undesirable tendencies, with high-resilient individuals able to accurately perceive a wide variety of social demands, and appropriately adjust responses. Conversely, low-resilient individuals are relatively unable to flexibly accommodate novel situations, and instead adopt a rigid stereotyped response-set.

A growing body of investigations have used cluster analyses (Asendorpf et al., 2001; Dubas, Gerris, Janssens, \& Vermulst, 2002; Hart, Atkins, \& Fegley, 2003), and more recently Latent Profile Analyses (Meeus, van de Schoot, Klimstra, \& Branje, 2011), to consider configurations of personality traits and their consistency with Block and Block's (1980) egoconstructs. An accumulation of evidence from typological research into personality has supported a three profile solution of personality reflecting resilient, overcontrol, and undercontrol types (Asendorpf et al., 2001; Caspi \& Silva, 1995; De Fruyt, Mervielde, \& Van Leeuwen, 2002; Hart, Hofmann, Edelstein, \& Keller, 1997; Meeus et al., 2011). This three profile solution is consistent with a theorised curvilinear relationship between extreme cases of either high or low levels of ego-control and low ego-resiliency (Asendorpf et al., 
2001). Specifically, Block and Block (1980) suggested that extreme levels of either high of low ego-control should result in a relatively inflexible responding pattern that is characteristic of low ego-resiliency.

In accordance with the proposed curvilinear relationship between ego-constructs, researchers have proposed that trait profiles identifying resilient individuals will be associated with high ego-resiliency, and moderate levels of ego-control, whereas overcontrol and undercontrol profiles will be associated with low ego-resiliency and extreme levels of high/low ego-control (Asendorpf et al., 2001; Caspi, 1998). Indeed, this pattern has been supported by some studies (Asendorpf \& van Aken, 1999; Robins, John, Caspi, Moffitt, \& Stouthamer-Loeber, 1996). Some researchers have subsequently concluded that the three profile solution is the minimum number of types necessary to constitute a generalizable solution (Robins et al., 1996).

Controversies and complexities in type development. As noted, the three profile model of trait configurations has gained broad support in typological investigations. However, researchers are still tackling a number of unresolved issues. Herzberg and Roth (2006) challenged the efficiency of the three profile solution, referencing concerns regarding the inconsistent configurations of personality traits across studies. The common labelling of such inconsistent configurations is a notable concern, as the practice disguises the issue of heterogeneity between studies. Strikingly, for example, the same pattern of low trait scores has been labelled overcontrol in some studies (Ekehammar \& Akrami, 2003; Berry, Elliott, \& Rivera, 2007; Steca, Allessandri, \& Caprara, 2010), and undercontrol in others (Dubas et al., 2002; Van Leeuwan, De Fruyt, \& Mervielde, 2004).

Adoption of the three profile model as valid is therefore premature. As noted previously, the three profile configuration of traits conflates both high and low control with low ego-resiliency, a step that has been described as a crucial conceptual flaw (Kremen \& Block, 1998). Alternatively, Letzring, Block and Funder (2005) suggest that the two constructs should be considered as theoretically distinct mechanisms. Individuals vary along both domains, such that ego-control will be most pertinent to individual differences in selfcontrol and self-regulation, and ego-resiliency will be most pertinent to individual differences in psychological adjustment and well-being. In line with these suggestions, 
researchers have proposed a four profile solution as a more precise theoretical alternative to the commonly adopted three profile solution (Barbaranelli, 2002; Gramzow et al., 2004).

Nevertheless, interactions between the two constructs may also occur. The constructs may function both independently and in conjunction to promote unique behavioural patterns (Letzring et al., 2005). The intricacies associated with these structures poses some notable issues for the identification of personality typologies, which typically address discrete groupings of individuals that show distinct patterns of personality. One solution is to calculate continuous-level data representing deviations from the central profile for each individual (Asendorpf, 2006; Asendorpf et al., 2001). Thus, rather than treating types as discontinuous, the relative degree of convergence across personality types may be considered, with the overlap between prototypical membership identified at fuzzy borders between profiles.

\section{Current Studies}

In the upcoming studies I will critically analyse the viability of a three profile model as a solution for type identification, and compare it to the alternative four profile model (Block \& Block, 1980; Letzring et al., 2005). I will also compare the validity of both Five and Six-Factor trait models as potential frameworks for profile development, and consider longitudinal stability, and relationships with pertinent outcome variables, to assess the construct validity of resultant types. Finally, I will assess the hypothesised relationship between profile configurations and ego-constructs using direct measures of the two ego-domains. A more detailed description of each study is provided below.

To provide rigorous tests of our models, I will use sound methodological and statistical strategies throughout these investigations. For instance, I will avoid biasing results by using large and representative samples. Additionally, I will employ Latent Profile Analysis to maximise the identification of coherent and meaningful types. Latent Profile Analysis is a multivariate statistical tool that assesses latent groupings through observed patterns of variable scores, and has been found to be superior to cluster analysis in several Monte Carlo studies (see Cleland, Rothschild, \& Haslam, 2000; McLachlan \& Peel, 2000) Although both cluster analysis and Latent Profile Analysis generate measurement and structural parameters, Latent Profile Analysis is unique in that it takes into account uncertainty of membership (error), and assigns class membership on the basis of probabilities (Nylund, 
Asparouhov, \& Muthen, 2007). As a consequence of the statistical advances reflected in Latent Profile Analysis, I will be able to employ novel statistical techniques to assess profile membership in terms of unique groupings, as well as the probability of belonging to each group or profile.

\section{General outline of studies}

Study 1. In the initial study, I investigated the competing three and four profile solutions of personality within a large, longitudinal, population-based sample. The current sample comes from historical data collected as part of the New Zealand Values and Attitudes Survey (NZAVS). Due to the incremental utility of including Honesty-humility as a sixth trait in personality investigations (Ashton \& Lee, 2008), I used the Six-Factor Model of personality as a basis for profile identification.

I developed the two models of personality profiles using Latent Profile Analysis within an initial cross-sectional sample (participants included in Wave 1 of the NZAVS). Measurements of model fit, as well as theoretical interpretability of trait configurations, were assessed and compared between solutions. I then considered the external validity of each solution using both continuous and categorical methodologies. Specifically, I considered the extent to which profiles effectively predicted relevant outcomes in adaptive/maladaptive and inhibited/uninhibited functioning using both mean-level differences, and degree of convergence with type-solutions. Finally, I assessed the longitudinal stability of profile membership, as well as invariance of profile structures, across the first three waves of the NZAVS.

Study 2. As a follow-up to study 1, I assessed the interpretability and replicability of the three and four profile solutions using the Five-Factor Model of personality as a basis for profile development. Additionally, I examined the incremental utility of using the Six-as opposed to Five-Factor Model of personality in profile identification. To enhance comparability across studies, I considered both three and four profile solutions using the same cross-sectional sample (Wave 1 of the NZAVS) and statistical techniques employed in Study 1.

First, I developed both three and four profile models using Latent Profile Analysis, and compared model fit and theoretical interpretability of the resultant structures. External validity was again assessed using relevant outcomes in adaptive/maladaptive and 
inhibited/uninhibited functioning using both mean-level differences, and degree of convergence with type-solutions. Finally, I assessed the comparative value of using the Sixas opposed to Five-Factor Model of personality in profile identification by comparing model fit and predictive ability with the results from study 1 .

Study 3. Based on the previous findings of studies 1 and 2, I directly assessed the proposed relationship between the four profile model identified in Study 1 with measures of Block and Block's (1980) ego-constructs. I targeted prototypical exemplars of each personality profile, who were identified by performing a preliminary Latent Profile Analysis on a combined sample of all respondents to the New Zealand Attitudes and Values Survey (NZAVS) across all waves of data collection. Only individuals with a high degree of convergence within their assigned profile were contacted for participation. Examinations of mean level differences in ego-resiliency and ego-control scores were then conducted to test the hypothesis that the four types identified within the four profile solution converge on high and low levels of ego-control and ego-resiliency. Supplementary analyses were also conducted to consider the relationships between the ego-control and ego-resiliency constructs. 

STUDY 1: Self-Regulation and Personality Profiles: Empirical Development, Longitudinal Stability, and Predictive Ability

\begin{abstract}
We used Latent Profile and Latent Profile Transition Analysis to empirically develop and compare competing models of personality profiles (three and four profile models). We do so using data from the New Zealand Attitudes and Values Study, a large longitudinal national probability sample of New Zealanders. Both three and four profile solutions demonstrated good fit and longitudinal stability. Trait configurations and predictive outcomes of the four profile model were the most interpretable in terms of the theoretical literature, as this solution mirrored the theoretical foundation of self-regulatory egoconstructs. This supported the interpretation of a four profile model as providing a useful distinction over and above the three profile model. We conclude that, compared to the three profile model, the four profile solution provides a better foundation to serve as a complementary approach to variable-centre research.
\end{abstract}




\section{Introduction}

A person-centred perspective on personality traits, as an important complement to the traditional variable-centred approach, has been revitalised by recent advances using a typological approach. The variable-centred approach focuses on using independent trait dimensions to describe individual differences between people. Augmenting this model, a person-centred approach considers the interactive effects of different personality characteristics working within the individual as a coordinated system. As argued by Asendorpf et al. (2001), personality traits may combine in particular ways within individuals to produce distinctive profiles or types. Such profiles are not random or idiosyncratic across individuals. Rather, individual personality types fall into a small number of separate clusters or categories in given samples.

Currently, the most well-replicated finding among researchers using the person-centred approach is the production of three personality profiles (Asendorpf et al., 2001; Caspi, 1998). However, it has been argued that this three profile model overlooks key distinctions between personality types, and that a four profile model provides a better foundation for a more substantive theoretical interpretation of the structures (Gramzow et al., 2004).

The present research uses Latent Profile Analysis and Latent Profile Transition Analysis, with a large representative sample of adults in New Zealand, to compare the theoretical and empirical interpretability of the three and four profile solutions of personality types. We will assess and discuss the trait configurations, cross-sectional replicability and longitudinal stability of these different solutions, and analyse how the profiles are linked to selfregulatory attitudes and behaviours in the domain of wellbeing, intergroup relations, selfconstraint and expressiveness. The theoretical backdrop and basis for this research are discussed next.

\section{Self-Regulation within an Interpersonal Context}

Self-regulation is described as the ability to organise and exercise control over one's behaviour. Block and Block (1980) developed a now prominent theoretical framework focusing on two properties that reflect individual differences in self-regulatory processes: ego-control and ego-resilience. Ego-control relates to the tendency to control or inhibit impulses, urges and desires, whereas ego-resilience refers to the elasticity, or flexibility of an individual's responding style. These two constructs are considered fundamental 
characteristics that govern reactions to the external environment and social interactions. It has been suggested that the emotional and behavioural patterns generated by these selfregulatory constructs could be represented by typological configurations of personality dimensions (Gramzow et al., 2004).

High levels of ego-control (overcontrol) can facilitate disciplined and directed behaviour, while low levels of ego-control (undercontrol) can promote comfort with ambiguity and inconsistency, as well as the desire to explore (Block \& Block, 1980). Both represent a mix of desirable and undesirable characteristics, which can promote positive and negative outcomes. Overcontrol has been associated with high frustration tolerance in children (Block \& Martin, 1955) as well as the ability to delay gratification in adolescence (Funder \& Block, 1989), but the decreased expressiveness of overcontrollers has also been associated with internalizing problems, such as anxiety, depression, and social withdrawal (Huey \& Weisz, 1997). Undercontrol has been associated with hardiness and ego-strength (Barron, 1953; Kobasa, 1979); however, the spontaneous sensation-seeking tendencies of undercontrollers can be maladaptive leading to erratic and impulsive behaviour, such as drug use (Block, Block \& Keyes, 1988), hyperactivity, aggression, and delinquency (Huey \& Weisz, 1997).

High levels of ego-resilience are associated with the flexible application of problemsolving strategies, and the ability to respond proactively to changes in the environment (Block \& Block, 1980). Unlike ego-control, ego-resilience is considered to range from desirable to undesirable tendencies as levels of ego-resilience decrease. In essence, the egoresilient individual is capable of accurately perceiving social demands within a wide variety of contexts, and adjusting behavioural responses accordingly. Individuals at the low end of this dimension (referred to as brittle) generally adopt stereotyped responses to novel situations, adapt badly to stress, and have difficulty coping with changes. Differences in egoresilience have been shown to predict competence in interpersonal relations and interpersonal functioning (Block \& Kremen, 1996) and psychological well-being (Letzring et al., 2005).

Number of types. According to Block and Block (1980), individuals may show variation along both of the two primary self-regulatory strategies: ego-resilience and ego-control. This suggests that there should be four primary profile structures (over/under control and 
high/low resilience), with some overlap between resilience and control strategies. However, as indicated, many researchers have identified three profiles of personality traits representing Block and Block's (1980) resilient, undercontrol and overcontrol egoconstructs, without identifying a fourth low-resilience profile group (Asendorpf et al., 2001; Caspi \& Silva, 1995; De Fruyt et al., 2002; Hart et al., 1997; Meeus et al., 2011).

This solution reflects Block's (1971) early suggestion that individuals with extremely high and extremely low ego-control should also score low in ego-resilience, a prediction which was supported by Robins et al. (1996), as well as Asendorpf and van Aken (1999), who found that both over- and under-control personality profiles in their three class solutions were associated with low levels of ego-resilience. Thus, it has been argued that three, rather than $2 \times 2$, primary personality profiles should be expected (Asendorpf et al., 2001). As a result of this apparent overlap between low ego-resilience (brittleness) with the polar extremes in levels of ego-control, brittle participants have rarely been directly represented in personality profiles (but see Barbaranelli, 2002; Gramzow et al., 2004). It is therefore not surprising that the overcontrol and undercontrol profiles described in the literature often resemble low-functioning over- and undercontrollers described by Block (1971) (see Barbaranelli, 2002; Berry et al., 2007; Robins et al., 1996) rather than the four profile structure suggested within the theoretical literature (Block \& Block, 1980).

However, the previous findings may be attributed to over-inclusiveness within the three profile solution, in which the low-resilient profile is included to varying degrees within the existing overcontrol and/or undercontrol profiles. This explanation has been suggested by Barbaranelli (2002), who compared participant distributions when using both the three and four structure solutions. In this previous analysis, the three profile solution included Resilient, Undercontrol, and Non-desirable/Overcontrol profiles, whereas the four profile solution distinguished Non-desirable, and Overcontrol profiles as distinct from one another. Due to the conflicting outcomes in previous investigations, as well as the apparent contradictions between Block and Block's (1980) foundations of ego-control and egoresilience and the dominant three profile findings, in the current research we analyse both the three and four profile organization of personality types in greater detail using the latest statistical analyses, and multiple stages of longitudinal data from large population-based samples. 
Typeness. According to Asendorpf (2006), prototype analysis assumes personality types are entirely discrete, and implies that there exist a few extremely potent genetic or environmental causes producing such a grouping. However, this implication has not been supported by previous investigations in personality theory and trait distribution (Asendorpf et al., 2001; Costa, Herbst, McCrae, Samuels, \& Ozer, 2002). Instead, Asendorpf (2006) calls for an approach that acknowledges variability within profiles and non-discrete borders between types.

Using this approach, members of each type would differ in terms of continuous levels of prototypicality, and outcomes could thus be analysed according to the extent to which each individual resembles the prototypical type member. We addressed this issue by applying novel statistical techniques in profile development which provides scores representing how closely the responses from any given participant match each given profile. In this manner, prototypically may be measured in terms of degree, and personality profiles may be converted into continuous-level variables.

Predictive validity of personality profiles. Consistent with the theoretical foundations of these personality profiles (Block \& Block, 1980), differences in ego-resilience should predict competence and amicability in interpersonal relations and interpersonal functioning (Block \& Kremen, 1996), in addition to well-being (Letzring et al., 2005). Consistently, higher-level cognitive capacities associated with ego-resilience, including sophisticated social reasoning, an internal locus of control, and the capacity to adopt flexible responding styles (Causadias, Salvatore \& Sroufe, 2012), have been associated with a decrease in both explicit and implicit prejudicial outcomes (Legault, Green-Demers, Grant, \& Chung, 2007). We therefore hypothesised that differences along the resilient domain would predict higher vs. lower levels of wellbeing, and more positive versus negative interpersonal functioning, including differences in prejudicial attitudes and Social Dominance Orientation, a dimension that reflects a competition-motivated drive for group dominance (Asbrock, Sibley, \& Duckitt, 2010; Duckitt \& Sibley, 2007).

Differences in ego-control, on the other hand, should predict interpersonal motivations related to inhibition, pleasure focus, and pursuit of new experiences. Overcontrollers are characteristically socially inhibited, uncomfortable with unfamiliar experiences, and even constrained to the point of denying themselves pleasure. At the other end of this 
continuum, undercontrollers are expressive even when it may be socially inappropriate, are pleasure-focused, comfortable with the unfamiliar, and have a tendency to engage in spontaneous sensation-seeking (Block, 2002). It seems likely that such dispositions should correspond with motivational values, specifically in regards to Schwartz's (1992) higherorder value dimensions "Openness to Change" and "Self-Enhancement". Openness to Change represents a motivational value structure that emphasises novelty, mastery, and the desire for pleasurable arousal, while Self-Enhancement emphasises hedonistic power motives, including social superiority, self-esteem and self-centred satisfaction. We therefore hypothesised that the pattern of lower inhibition and higher comfort with the unfamiliar associated with undercontrollers should predict higher Openness to Change and SelfEnhancement values, whereas the inhibitory and cautious nature of overcontrollers would make them less likely to endorse these same values.

Personality variables. The trait dimensions on which personality profiles are based inevitably influence the types that emerge. As Asendorpf et al. (2001) point out, different types may be found for different sets of trait dimensions, and more types may be found when using more traits (or factors). This has particular significance when considering the current debate in the literature regarding the optimal number of personality factors. A large body of research identifies the Five-Factor model of personality as core to the assessment of personality (Digman, 1990; John \& Srivastava, 1999; McCrae \& John, 1992), whereas a growing number of researchers have pointed to the utility of a sixth personality factor, Honesty-humility, on top of the Big Five factors (Openness, Agreeableness, Conscientiousness, Extraversion and Neuroticism). Honesty-humility is defined by such qualities as sincerity and fairness versus conceit and greed (Ashton \& Lee, 2008). This set of six factors (HEXACO) is similar to the Five-Factor Model, with a slight difference in the Emotional Stability (Neuroticism reversed) and Agreeableness factors.

The incremental utility of this additional factor is apparent in the ability of the HEXACO model to predict certain personality-related constructs, including Materialism (Ashton \& Lee, 2008), Delinquency, Machiavellianism, and Narcissism (Lee \& Ashton, 2005; Lee, Ashton, \& de Vries, 2005), as well as other variables from the Supernumerary Personality Inventory (SPI; Paunonen, 2002), an inventory designed to assess a variety of important personality characteristics that are largely "beyond the Big Five" (Lee et al., 2005). Despite 
the modifications to the Agreeableness and Emotional Stability variables within the HEXACO model, the advantages of this model appears to be primarily due to the Honesty/humility factor, as analyses conducted by adding this factor to the traditional Five-Factor Model have been shown to demonstrate similar multiple correlations as those obtained using the HEXACO model (Ashton \& Lee, 2008). Based on the theoretical plausibility of the six-factor model, and its superior predictive ability, we used the six-factor model in the current research on self-regulatory trait-profiles.

\section{Current Analyses}

The present study aimed to address some major controversies in the development and interpretation of personality profiles using a large, longitudinal, population-based sample. Based on the previous literature and theoretical justifications, we focused primarily on issues concerning the identification and comparison of three or four profile solutions of trait-configurations, and tested each model's validity by comparing its fit, structural interpretability, and longitudinal stability using similar analytic techniques to those by Meeus et al. (2011). This previous investigation employed Latent Profile and Latent Transition techniques on an adolescent sample, and identified a three profile model. Moreover, the assessed relationship between Resilient and Overcontrol profiles with outcome variables (specifically, change in generalised anxiety disorder) within their study demonstrated some evidence supporting the decision to interpret the Resilient and Overcontrol profiles and label them accordingly. However, the Undercontrol profile demonstrated particularly poor stability, and the interpretation of this profile was not supported by the ability of the profile to predict any relevant outcomes. We investigated this question more thoroughly by testing the potential relationship of each profile within both three and four profile models, along with relevant outcome variables, to assess the interpretability and predictive ability of both solutions.

We predicted that the resultant profiles would be interpretable in terms of ego-control and ego-resilience; however we remained open to the possibility of a best-fitting solution consisting of either three or four profiles. Consistent with the previous literature, we expected that a three profile model would reflect resilient, brittle/overcontrol, and brittle/undercontrol solutions (Asendorpf \& van Aken, 1999; Robins et al., 1996), while a four profile model would reflect differences according to high and low attributes of both 
resilience and control. Additionally, to the extent that configurations of personality traits are consistent with Block and Block's (1980) self-regulatory constructs, we expected the personality profiles to be relatively stable across time, and to correspond with both desirable and adaptive outcomes (in the case of ego-resilience) and with self-indulgent and low-inhibitory outcomes (in the case of ego-control). Of particular interest theoretically are the functions of these personality profiles as self-regulatory personality systems within interpersonal domains.

\section{Method}

\section{Sample}

We analysed responses from 6518 individuals who participated in Wave 1 of the New Zealand Values and Attitudes Survey (NZAVS). Our longitudinal dataset was comprised of 4764 participants who responded to both the first wave of data collection, as well as a minimum of one of the additional two waves. Females represented the majority of participants in the Time 1 (59.3\%) and longitudinal (60.8\%) datasets. Mean age of the respondents was $47.91(S D=15.73)$ in the Time 1 dataset, and $49.44(S D=15.28)$ in the longitudinal data set.

Sampling procedures and sample properties. Wave 1 (2009) of the NZAVS contained responses from 6,518 participants sampled from the 2009 New Zealand electoral roll. The electoral roll is publicly available for scientific research and in 2009 contained 2,986,546 registered voters. This represented all citisens over 18 years of age who were eligible to vote regardless of whether they chose to vote, barring people who had their contact details removed due to specific case-by-case concerns about privacy. The sample frame was spilt into two parts. Sample Frame 1 constituted a random sample of 25,000 people from the electoral roll (4,060 respondents). Sample Frame 2 constituted a second random sample of a further 10,000 people from the electoral roll (1,609 respondents).

Wave 2 (2010) of the NZAVS retained 4,423 from the initial Wave 1 (2009) NZAVS sample of 6,518 participants, and included an additional 20 respondents who could not be matched to the Wave 1 participant database (a retention rate of $67.9 \%$ over one year). Wave 3 (2011) of the NZAVS contained responses from 6,884 participants (3,918 retained, 2,965 new participants). This Wave retained 3,916 from the initial Wave 1 national probability sample (a $60.1 \%$ retention rate over two years). Three participants who joined at 
Wave 2 were also retained. Participants were posted a copy of the questionnaire, with a second postal follow-up two months later. Participants who provided an email address were also emailed and invited to complete an online version if they preferred.

\section{Measures $^{1}$}

Personality: Mini-IPIP6. The 6-factor model was used in the current analyses. Participants completed the 5 factor 20-item Mini-IPIP (Donnellan, Frederick, Oswald, \& Lucas, 2006), as well as four items measuring the sixth trait, Honesty/humility, which was developed and validated by Sibley et al. (2011). Each personality item consists of a phrase evaluating a trait-relevant behaviour (e.g., 'I have a good word for everyone'), and is measured on a 7-point Likert scale. Reverse-items were recoded and scores for individual items were averaged to produce a total score for each factor. An exploratory factor analysis (Appendix A) demonstrated that items loaded strongly onto the appropriate factors, while internal consistency reliability values for each factor were high, given the small number of items identifying them: Openness ( $a=.67)$, Agreeableness $(a=.67)$, Extraversion $(a=.71)$, Conscientiousness $(a=.65)$, Emotional Stability $(a=.64)$, and Honesty/humility $(a=.78)$.

Outcome variables. For detailed information regarding item development, refer to Sibley (2009). All items were measured on a 7 point Likert scale, excluding the two 'Satisfaction with Life' items, which were measured on a 10 point Likert scale, as well as all of Schwartz's higher-order value items, which were measured on a 9 point Likert scale.

Subjective wellbeing. Nine items targeting different facets of subjective wellbeing were measured (details below). An internal consistency value was calculated for all well-being items, demonstrating a high score $(a=.83)$. Z-scores were calculated for all nine items, which were then averaged to provide a total subjective-wellbeing score.

a. Self-esteem: three items from the 10-item Rosenberg (1965) Self-Esteem scale.

b. Personal-wellbeing: four items from the Personal Wellbeing Index (Cummins, Eckersley, Pallant, van Vugt, \& Misajon, 2003).

c. Satisfaction with life: two items from the Satisfaction with Life scale (Diener, Emmoms, Larsen, \& Griffin, 1985).

\footnotetext{
${ }^{1}$ For scale items and instructions, view Appendix B
} 
Perceived quality of interethnic relations. The perceived quality of interethnic relations was measured using a range of variables. These include single items measuring the perceptions of realistic threat (adapted from Bobo (1998)) and symbolic threat (adapted from Stephan et al. (2002)) to New Zealanders in general, which were repeated four times in reference to different ethnic groups (Maori, NZ Europeans, Pacific Islanders, Asians). Internal consistency reliability was high for the four single-item measures for both realistic $(a=.83)$ and symbolic $(a=.85)$ threat, and items were averaged to produce total scores or realistic and symbolic threat.

Three additional single-item measures were then included (details below). The internal reliability score for the resultant 5 variables was calculated and, given the few items, is good $(a=.66)$. All items were reverse coded, and the 5 variables were averaged to produce a total score.

a. Racial essentialism: from the Lay Theory of Race Scale developed by No et al. (2008).

b. Intergroup anxiety: adapted by Sibley and Barlow (2009; cited in Sibley, 2009) from Stephan and Stephan (1985).

c. Race-based rejection sensitivity: adapted by Sibley and Barlow (2009; cited in Sibley, 2009) from Shelton and Richeson (2005).

Social dominance orientation. Social Dominance Orientation was measured using six items from the 16-item Social Dominance Orientation scale reported in Sidanius and Pratto (1999). Internal consistency reliability for this scale $(a=.69)$ was good.

Self-enhancement and openness to change values. The shortened 12-item measure of Schwartz's (1992) values, adapted by Stern, Dietz, and Guagnano (1998), was included. For the current analyses, we only consider the 3 Openness to Change and the 3 SelfEnhancement items. Given the small number of items, internal consistency reliability was high for both Openness to Change $(a=.73)$, and Self Enhancement $(a=.61$ ) values.

\section{Procedure}

Similar to a previous investigation by Meeus et al. (2011) we developed the personality profiles using a Latent Profile Analysis (LPA) of participants' responses to trait measures using MPLUS software (Muthèn \& Muthèn, 2012). This is a multivariate statistical model which effectively functions as a confirmatory cluster analysis. LPA is based on measurement theory that a latent grouping variable can be inferred from a set of indicators, such that 
individuals in each group share common patterns of variable scores. This model generates fit statistics as well as significance tests, and takes into account measurements of uncertainty (error) before ultimately assigning participants to different profiles. We focused our Latent Profile Analyses on comparing the fit of both three and four profile models at the first wave of data collection. We then compared the interpretability of the three and four profile solutions by examining trait configurations within each structure, and considered the similarity of participant distribution across models by calculating frequency of participant overlap between three and four profile solutions.

When conducting a Latent Profile Analysis, each participant is assigned 'class membership probability' scores, which indicates the probability of being assigned to each potential class. Members of each type therefore differ in terms of continuous levels of prototypicality, allowing us to use traditional regression analyses to assess the predictive ability of each solution. Probability of class membership for each participant within each solution ranges from $0.00-1.00$, and the sum of each participant's probabilities must sum to 1.00. In this manner, the scores are completely dependent. Because probabilities are not independent across all four profiles, these profiles cannot be combined to predict outcomes.

Demographic information was first analysed by regressing probability of class membership for each personality profile on age, sex and income simultaneously. After controlling for demographic information, we regressed each of the three and four solution personality profiles on subjective wellbeing, perceived quality of interethnic relations, Social Dominance Orientation, and Self-Enhancement and Openness to Change values. We then conducted a series of one-way between-subjects ANOVAs to compare the mean-level differences relating to each of these outcome variables in both three and four profile solutions using more traditional type-based techniques.

Before considering the stability of personality profiles, we assessed the current dataset for systematic attrition, which, if not corrected, can bias the analyses (Little \& Rubin, 2002). We then considered trends in attrition and imputed missing variables in the longitudinal dataset using Estimation Maximization procedures in all cases where we had participant information for two or more waves of data collection. Missing scores were not imputed in instances where we had limited information, specifically for those participants who responded exclusively at the first wave of data collection. 
We then performed a Latent Profile Transition Analysis (LPTA) to assess the stability of both three and four profile solutions over a one-year and two-year period. LPTA represents a longitudinal extension of the LPA, where the stability or change in profile membership over time may be observed. Using this procedure, membership in latent profiles are estimated at each wave of data collection. Transition probabilities are then freely estimated to reflect the probability of transitioning from each latent profile to another across intervals. Following this, we assessed the measurement invariance of profile configurations across intervals by constraining item-response probabilities, and comparing the model fit of both nested models.

\section{Results}

As recommended by several authors (Bauer \& Curran, 2004; Nagin \& Tremblay, 2005), we incorporated multiple analyses, along with theoretical and practical considerations, when considering our models' fit and interpretability. Two fit statistics reported by MPLUS include the Bayesian Information Criterion (BIC; Schwarz, 1978) and the Bootstrap Likelihood Ratio Test (BLRT; McLachlan \& Peel, 2000). The BIC is used to compare across two different models, with smaller values indicating better model fit. Specifically, a difference in $\mathrm{BIC}$ of 10 is considered strong evidence in favour of the model with the smaller BIC value (Raftery, 1995). Similarly, the BLRT solution produces a $p$ value to represent the likelihood that the solution being investigated is superior to a hypothetical solution with one less class.

BLRT scores demonstrated a better fit for the three profile solution as compared to a hypothetical two profile solution, whereas the four profile solution demonstrated a better fit than the three profile solution (in each case, $p<.001$ ). Furthermore, the difference between the BIC of the four profile solution was lower (difference of 247.52) than the BIC of the three profile solution. These results suggest that the fit of the four profile solution is superior to the fit of the three profile solution. However, we also found that these scores continued to show an increase in fit upon adding additional profiles, even after additional profiles were no longer interpretable and membership within profiles dropped well below $1 \%$. We suggest that these findings could be understood as resulting from an overpowering of the current sample, and therefore cannot provide robust evidence for either three or four profile solution (see also Huang, n.d.; Marsh, Lüdtke, Trautwein, \& Morin, 2009). 
Next, we considered the strength of profile classification within each solution by calculating the average posterior class membership and entropy. The classification of individuals into latent profiles is based on their estimated posterior probabilities given the individual's response pattern on the observed variables. An individual's estimated posterior probabilities within each profile range from $0-1$, with 0 indicating no fit, and 1 indicating a perfect fit. The sum of an individual's posterior probabilities when considering their potential placement within each possible profile is equal to 1 . An extension of these scores is the average posterior class membership probability. This is calculated by averaging the class membership probability of all individuals within each class after they have been ultimately assigned a given class, and represents the strength of individual profiles by demonstrating the within-profile potential for misclassification. In practice, misclassification is inevitable, however, the generally recognised rule of thumb for acceptable profile classification is when the average posterior class membership probability is 0.70 or greater (Nagin, 2005).

Entropy is calculated by assessing how many participants are ultimately assigned to each profile, and comparing this to the sum of all of the participants' cumulative posterior membership probabilities within each potential profile, regardless of final profile assignment (Clark \& Muthèn, 2009). The entropy reported in MPLUS is the relative entropy of a model, measured between $[0,1]$, with higher values indicating less overall overlap in membership classification (and therefore indicating more discrete and better fitting personality types). Although there is no clear cut-off point for the value of entropy to ensure a minimum level of good classification, Clark and Muthèn (2009) suggests an interpretation of 0.8 as high, 0.6 as medium and 0.4 as low entropy.

Table 2 presents fit statistics for solutions ranging from three to four classes at the first wave of data-collection. In each solution, the entropy is medium, demonstrating some overlap between classes. A medium entropy is acceptable for the current model as entirely discrete classes were not anticipated, and some degree of overlap is predicted in the theory (Asendorpf, 2006). Furthermore, the average posterior class membership probabilities are adequately robust in both solutions. These numbers suggest that, despite some interpretable overlap between profiles, the mean convergence with three and four prototypical profile solutions is acceptable. 
Prototypical trait configurations of the three and four profile solutions were developed by calculating mean scores of each personality trait for all participants ultimately assigned to each profile. Figures $1 \mathrm{a}$ and $1 \mathrm{~b}$ show the resultant prototypical profile structures among Wave 1 participants. Both three and four profile models were consistent with the previous literature: in both models, the resilient profile was characterised by high (or desirable) mean values on each personality trait, while the overcontrol profile exhibited low Extraversion and Openness (see Asendorpf et al., 2001; Robins et al, 1996; Roth \& von Collani, 2007).

Table 2

Six-Factor Model (SFM) personality profiles:

Fit statistics for 3 and 4 profile solutions

\begin{tabular}{lccccc}
\hline & & \multicolumn{4}{c}{ Average Probability of Class Membership } \\
\cline { 3 - 6 } & Entropy & Profile 1 & Profile 2 & Profile 3 & Profile 4 \\
\hline 3 Profile Solution & .51 & .75 & .76 & .80 & -- \\
4 Profile Solution & .54 & .70 & .75 & .71 & .74 \\
\hline$n=6,518$ & & & & &
\end{tabular}

Conversely, the undercontrol profile demonstrated substantial change across solutions. In the three profile solution (Figure 1a), this third pattern of traits exhibited generally moderate scores on all traits, but comparably higher scores in Extraversion and Openness, and lower scores in Honesty/humility, than the overcontrol profile. In the four profile solution (Figure 1b), the undercontrol profile retained its characteristic scores on Extraversion, Openness, and Honesty/humility, along with a mix of high to low scores on other traits, whereas the brittle profile was characterised by exclusively low (or undesirable) scores on all traits (see Barbaranelli, 2002; Gramzow et al., 2004).

Despite the conceptual interpretability of Conscientiousness as differentiating between high and low self-regulatory strategies, we found no notable differences in levels of Conscientiousness between overcontrol and undercontrol profiles in either three or four profile solutions. However, in both current solutions, undercontrollers presented with low, and overcontrollers with high, Honesty/humility. This trait possesses features of restraint/inhibition, as well as tendencies towards self-monitoring (Ashton \& Lee, 2005), that may account for much of the inhibitory/reactive differences between over and undercontrol strategies that may otherwise be accounted for by Conscientiousness. Further investigations conducting a thorough head-to-head comparison of personality profiles using 
both Five- and Six-Profile models must be conducted to consider this possibility in greater detail.

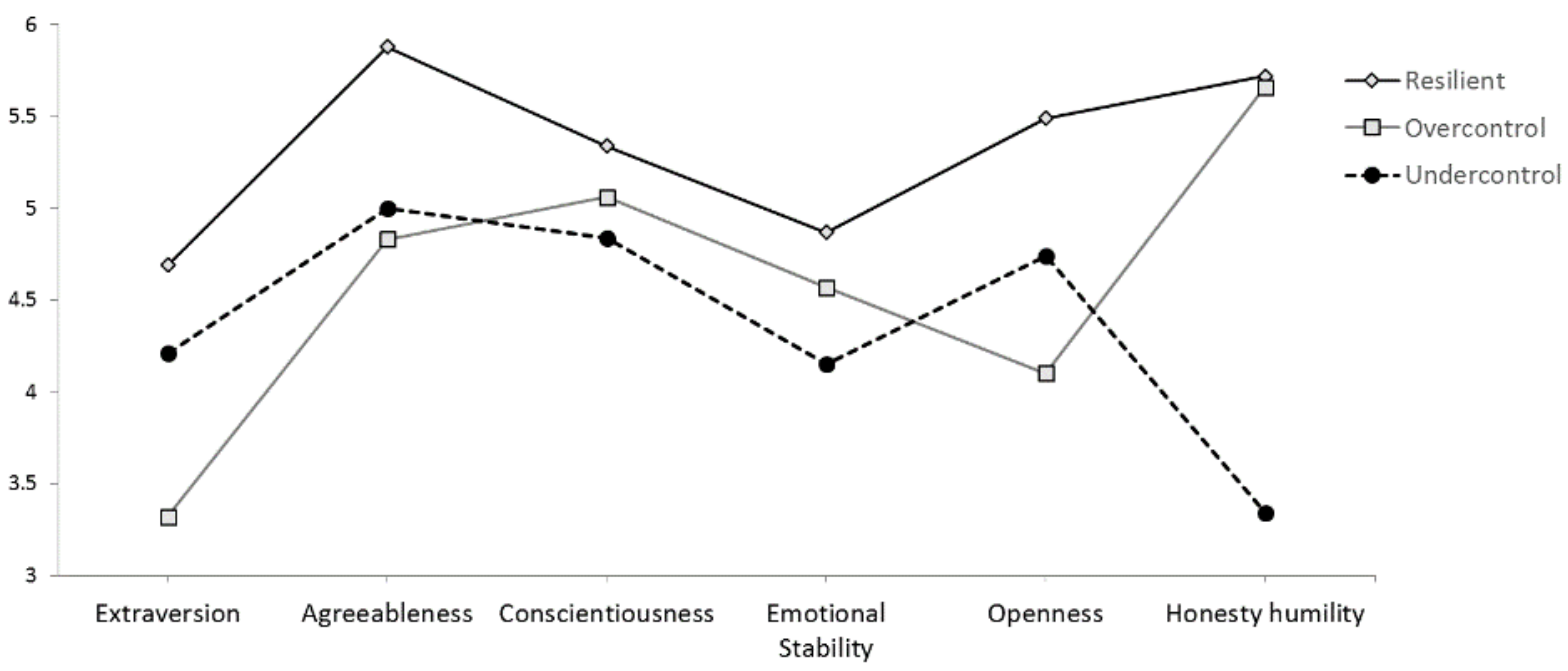

Figure 1a: Three Profile solution of personality traits (Six-Factor Model)

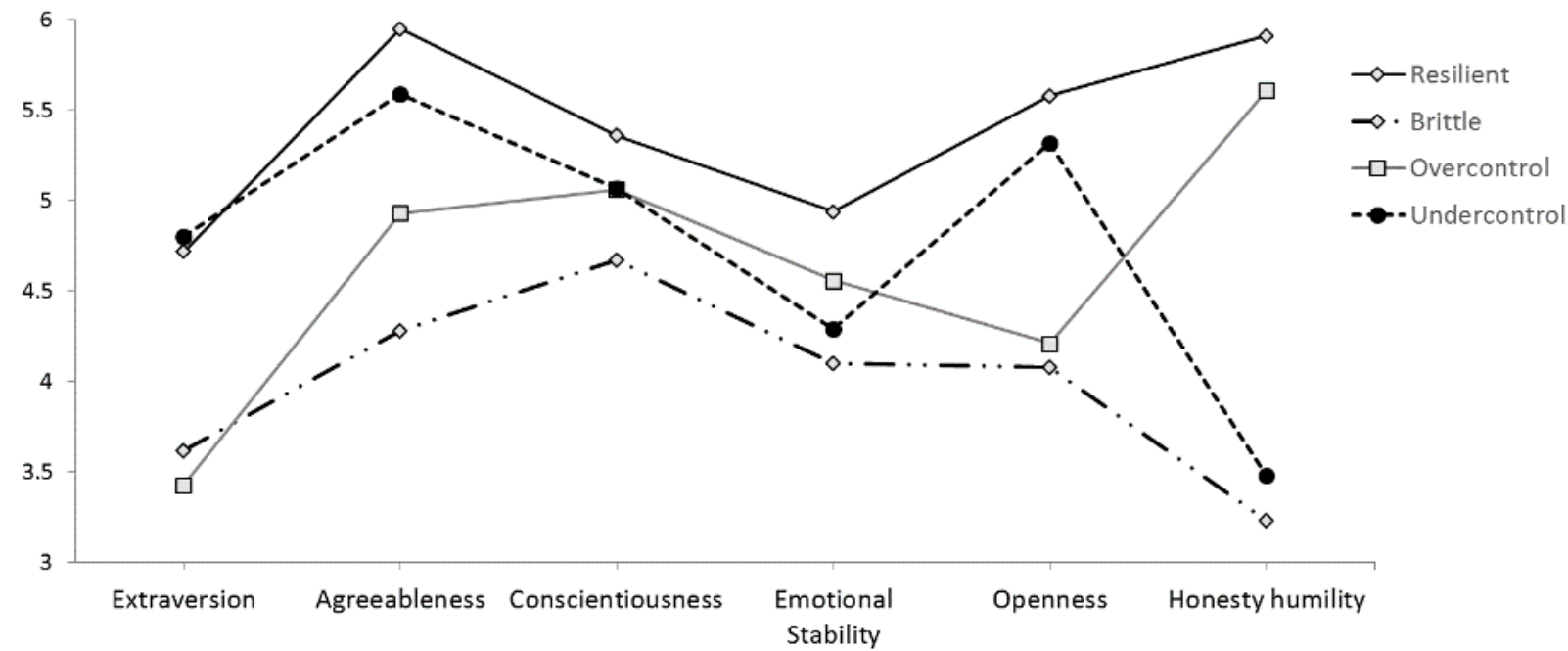

Figure 1b: Four Profile solution of personality traits (Six-Factor Model)

We then calculated the proportion of the total sample represented within each profile in the three and four profile models. For profiles to represent adequately interpretable solutions, we would be uncomfortable with accepting solutions that dropped below $5 \%$ of the total sample. As demonstrated in Table 3, each profile represented over $10 \%$ of the total sample in both solutions. Moreover, participant distribution further suggested that the four profile undercontrol and brittle profiles may both be offshoots of the three profile undercontrol solution. Specifically, the proportion of resilient and overcontrol profiles 
shifted only marginally across solutions, whereas the undercontrol configuration in the three profile solution dropped dramatically upon the inclusion of a fourth (brittle) profile.

Table 3

Participant distribution in SFM personality profiles:

Percent of Total Sample

\begin{tabular}{lcccc}
\hline & Resilient & Overcontrol & Undercontrol & Brittle \\
\hline 3 Profile Solution & 34.6 & 37.5 & 27.9 & -- \\
4 Profile Solution & 27.0 & 43.3 & 17.3 & 12.3 \\
\hline$n=6,518$ & & & &
\end{tabular}

Judging from comparisons of the configurations of traits within both three and four profile models, two different interpretations may be developed: the four profile solution may reflect unnecessarily precise subcategories of undercontrol, or the three profile solution may ignore a necessary distinction between 2 distinct profiles. Although this distinction may seem slight, it is necessary to investigate which of these two different explanations best explains the configurations in order to place the resultant profiles within a clearer theoretical context.

To further consider comparisons between participant distributions within the three and four profile models, we calculated the proportion of participants that co-occurred within each of the three and four profile solutions (Table 4). Membership within resilient and overcontrol profiles replicated well in both models and, as expected, participants from the three solution undercontrol profile were primarily represented within both the four solution brittle and undercontrol profiles.

Table 4

Participant distribution in SFM personality profiles:

Percent of profile overlap

\begin{tabular}{|c|c|c|c|c|}
\hline & & \multicolumn{3}{|c|}{ Three profile solutions } \\
\hline & & Resilient & Overcontrol & Undercontrol \\
\hline \multirow{4}{*}{ 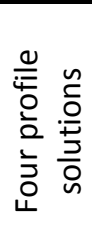 } & Resilient & 100 & 0.00 & 0.00 \\
\hline & Overcontrol & 10.0 & 84.4 & 5.6 \\
\hline & Undercontrol & 18.5 & 0.00 & 81.5 \\
\hline & Brittle & 0.00 & 7.4 & 92.6 \\
\hline
\end{tabular}

$n=6,518$

For the four profile solution to provide a useful distinction between types over and above the three profile model, outcomes relating to brittle and undercontrol participants must be interpretable and theoretically justifiable. In line with the theoretical foundation of 
four profile model (Block \& Block, 1980), we would expect the four profile undercontrol solution to demonstrate low-inhibitory outcomes, compared to the overcontrol solution, which would reflect high-inhibitory outcomes. Consistently, the four profile resilient and brittle solutions would be expected to reflect differences along high/low adjustment, without distinct trends towards high/low inhibition. Alternatively, for the three profile solution to be interpretable in terms of the curvilinear relationship between low-adjustment and both extremes of the control dimension (Asendorpf \& van Aken, 1999), outcomes relating to both three solution over- and undercontrol profiles should reflect high- vs lowinhibition, along with maladjusted outcomes in each case.

We therefore assessed both sets of solutions as they related to adjustment and inhibition outcome variables. First, we considered the relationships between profiles and demographic variables. As presented in Table 5, demographic information demonstrated some relationships with personality profiles. In both three and four profile models, being female was positively associated with the resilient profile, and age was positively associated with the overcontrol profile. Age was negatively associated with both brittle and undercontrol profiles in the four profile model, whereas being male was positively related to the four solution brittle profile, and the three solution undercontrol profile. Income had no distinct association with any profile.

Table 5

SFM personality profile membership regressed on demographic variables: Standardised beta weights

\begin{tabular}{|c|c|c|c|c|c|}
\hline \multicolumn{6}{|c|}{ Three profile solution } \\
\hline & & Resilient & \multirow{2}{*}{$\begin{array}{c}\text { Overcontrol } \\
-.02\end{array}$} & \multicolumn{2}{|l|}{ Undercontrol } \\
\hline Sex & B & $.17^{* *}$ & & $-.14^{* *}$ & --- \\
\hline Age & B & $.04^{*}$ & $.23 * *$ & $-.26 * *$ & --- \\
\hline Income & $\mathrm{B}$ & $.08 * *$ & -.01 & $-.07 * *$ & --- \\
\hline \multicolumn{6}{|c|}{ Four profile solution } \\
\hline & & Resilient & Overcontrol & Undercontrol & Brittle \\
\hline Sex & B & $.16^{* *}$ & -.01 & -.03 & $-.18 * *$ \\
\hline Age & B & $.06 * *$ & $.23 * *$ & $-.24 * *$ & $-.14 * *$ \\
\hline Income & B & $.07^{* *}$ & .00 & -.01 & $-.09 *$ \\
\hline \multicolumn{6}{|c|}{$n=6,518$} \\
\hline \multicolumn{6}{|c|}{ Sex: Male coded 1; Female coded 2} \\
\hline
\end{tabular}

Standardised regression coefficients for each profile/DV relationship are listed in Table 6. As predicted, the resilient profile in both three and four profile solutions was associated 
with adaptive outcomes, including positive relationships with wellbeing and the perceived quality of interethnic relations, and a negative relationship with Social Dominance Orientation. Additionally, the overcontrol profile within both models was associated with inhibitory outcomes, as characterised by negative relationships with both Openness to Change and Self-Enhancement Values, but was not associated with measured maladaptive outcomes. This is consistent with Block and Block's (1980) perspective of ego-control reflecting neither an inherently adaptive or maladaptive self-regulatory style.

Table 6

Beta weight of SFM profile membership regressed on outcome variables

\begin{tabular}{|c|c|c|c|c|c|c|}
\hline & Profile & $\begin{array}{l}\text { Personal } \\
\text { Wellbeing }\end{array}$ & $\begin{array}{c}\text { Perceived } \\
\text { Interethnic } \\
\text { Relations }\end{array}$ & $\begin{array}{c}\text { Social } \\
\text { Dominance } \\
\text { Orientation }\end{array}$ & $\begin{array}{c}\text { Openness to } \\
\text { Change } \\
\text { Values }\end{array}$ & $\begin{array}{c}\text { Self } \\
\text { Enhancement } \\
\text { Values }\end{array}$ \\
\hline \multirow{3}{*}{$\begin{array}{l}\frac{0}{i \frac{\pi}{0}} \\
\frac{0}{\alpha} \\
\dot{a}\end{array}$} & Resilient & $.27^{* *}$ & $.36^{* *}$ & $-.25^{* *}$ & $.20 * *$ & $-.12 * *$ \\
\hline & Overcontrol & $-.09 * *$ & $-.04 * *$ & $.03^{*}$ & $-.29 * *$ & $-.23 * *$ \\
\hline & Undercontrol & $-.18^{* *}$ & $-.30 * *$ & $.20 * *$ & $.07 * *$ & $.33^{* *}$ \\
\hline \multirow{4}{*}{$\begin{array}{l}\frac{0}{i \frac{0}{0}} \\
\frac{0}{2} \\
\frac{1}{4}\end{array}$} & Resilient & $.27^{* *}$ & .35 & $-.25 * *$ & $.18^{* *}$ & $-.14 * *$ \\
\hline & Overcontrol & $-.07 * *$ & .00 & .02 & $-.27^{* *}$ & $-.24 * *$ \\
\hline & Undercontrol & $-.05 * *$ & $-.11^{* *}$ & $.08^{* *}$ & $.18^{* *}$ & $.30 * *$ \\
\hline & Brittle & $-.20 * *$ & $-.33 * *$ & $.21 * *$ & $-.05 * *$ & $.18^{* *}$ \\
\hline
\end{tabular}

$n=6,518$

Note: All regressions are conducted after controlling for demographic variables Predictors entered individually

Outcomes were also consistent with the interpretation of the remaining solutions as reflecting a brittle/undercontrol profile in the three profile solution, and separate brittle and undercontrol profiles in the four profile solution. The outcomes related to brittle and undercontrol profiles in the four profile solution were directly contrasted to the resilient and overcontrol outcomes, respectively. Specifically, the four profile model undercontrol solution was positively associated with both Openness to Change and Self-Enhancement Values, with no distinct trend relating to adjustment, whereas the brittle profile was related to maladaptive outcomes including negative relationships with wellbeing and the perceived quality of interethnic relations, a positive relationship with Social Dominance Orientation, and (to a lesser extent) Self-Enhancement Values. Comparably, the three solution undercontrol profile was associated with maladaptive outcomes and Self-Enhancement values, with no relationship to Openness to Change Values. As will be discussed, a profile 
that exhibits both high levels of Openness to Change, as well as Self-Enhancement values, is distinctively interpretable in terms of a four profile model.

A series of one-way between-subjects ANOVAs were conducted comparing profiles within both three and four profile solutions to examine mean-level differences relating to each of the five outcome variables. Due to heterogeneous variances between profiles (demonstrated using Levene's test of homogeneity of variance), Welch's robust test of equality of means was used to compare means across profiles. Results (Tables 7a and 7b) confirmed that there was a mean-level difference relating to each outcome variable between profiles in both three and four profile solutions.

Table 7a

SFM four profile solution:

One-way ANOVAs predicting mean-level differences in outcome variables

\begin{tabular}{lccccccccccc}
\hline \multirow{2}{*}{ Dependent variables } & \multicolumn{2}{c}{ Resilient } & \multicolumn{3}{c}{ Brittle } & \multicolumn{2}{c}{ Overcontrol } & \multicolumn{2}{c}{ Undercontrol } \\
\cline { 2 - 9 } & $M$ & $S D$ & $M$ & $S D$ & $M$ & $S D$ & $M$ & $S D$ & \\
\hline Personal Wellbeing & .25 & .61 & -.29 & .68 & $-.05^{\mathrm{a}}$ & .67 & $-.06^{\mathrm{a}}$ & .66 & $153.41^{*}$ \\
Social dominance orientation & 2.26 & .91 & 3.05 & .96 & $2.64^{\mathrm{b}}$ & .94 & $2.70^{\mathrm{b}}$ & .96 & $147.92^{*}$ \\
Percieved Interethnic Relations & 5.17 & .81 & 4.42 & .78 & $4.79^{\mathrm{c}}$ & .78 & $4.78^{\mathrm{c}}$ & .81 & $181.84^{*}$ \\
Self-Enhancement Values & $3.45^{\mathrm{d}}$ & 1.35 & 4.28 & 1.29 & $3.43^{\mathrm{d}}$ & 1.34 & 4.45 & 1.26 & $241.81^{*}$ \\
Openness to Change Values & 5.12 & 1.22 & 4.66 & 1.32 & 4.42 & 1.35 & 5.26 & 1.15 & $176.89^{*}$
\end{tabular}

Note. $M=$ Mean; $S D=$ Standard Deviation. ${ }^{*} p<.001$.

Means with similar superscripts did not differ significantly $(p>.05)$.

Table 7b

SFM three profile solution:

One-way ANOVAs predicting mean-level differences in outcome variables

\begin{tabular}{lcccccccc}
\hline \multirow{2}{*}{\multicolumn{1}{c}{ Dependent variables }} & \multicolumn{2}{c}{ Resilient } & \multicolumn{2}{c}{ Overcontrol } & \multicolumn{2}{c}{ Undercontrol } & \multirow{2}{*}{ Welch's $F$} \\
\cline { 2 - 7 } & $M$ & $S D$ & $M$ & $S D$ & $M$ & $S D$ & \\
\hline Personal Wellbeing & .21 & .62 & -.06 & .67 & -.18 & .67 & $203.23^{*}$ \\
Social dominance orientation & 2.31 & .92 & 2.66 & .94 & 2.87 & .97 & $185.42^{*}$ \\
Percieved Interethnic Relations & 5.12 & .81 & 4.77 & .78 & 4.60 & .81 & $226.70^{*}$ \\
Self-Enhancement Values & 3.54 & 1.34 & 3.40 & 1.35 & 4.36 & 1.29 & $307.79^{*}$ \\
Openness to Change Values & 5.10 & 1.21 & 4.36 & 1.37 & 4.96 & 1.25 & $208.85^{*}$ \\
\hline
\end{tabular}

Note. $M=$ Mean; $S D=$ Standard Deviation. ${ }^{*} p<.001$.

All means differed significantly $(p<.01)$. 
Tukey post-hoc analyses were consistent with our previous interpretations of the four profile solution (Table $7 a$ ) as representing a $2 \times 2$ solution of high/low resilience/control. Specifically, the resilient profile demonstrated the highest, and the brittle profile the lowest, levels of adjustment on the three adjustment outcomes (Personal Wellbeing, Perceived Interethnic Relations, and Social Dominance Orientation). Both over- and under-control profiles were associated with moderate scores on these adjustment outcomes, and did not significantly differ from one another.

Additionally, the undercontrol profile demonstrated the highest scores on both SelfEnhancement and Openness to Change values, whereas the overcontrol profile demonstrated the lowest scores on these values (resilient and overcontrol did not significantly differ in regards to Self-Enhancement values). Resilient and brittle profiles demonstrated mixed trends in self-regulatory strategies. The resilient profile scored higher on Openness to Change, and lower on Self-Enhancement, as compared to the brittle profile. Taken together, these outcomes are consistent with the resilient and brittle profile as representing primarily well- and mal-adjusted participants, respectively, whereas overcontrol and undercontrol solutions reflected variation in inhibitory tendencies.

Comparably, mean-level differences within the three profile solution (Table 7b) demonstrated a less precise convergence with outcome variables. Resilient and undercontrol profiles mirrored the adjusted and maladjusted trends associated with resilient and brittle profiles within the four profile solution. Comparably, the overcontrol profile demonstrated high inhibitory tendencies reflected by low Self-Enhancement and Openness to Change values. These findings lend support to our previous suggestion that the three profile undercontrol solution reflects primarily maladaptive, rather than lowinhibitory, tendencies. The differential predictive ability of these competing models further demonstrates that the current three profile solution ignores a necessary distinction between low-resilience and low-control tendencies.

Before considering the stability of the three and four profile models, we evaluated attrition across waves using Little's MCAR test (Little \& Rubin, 2002). We found a significant violation of MCAR, $\chi^{2}(114)=278.08, p<.001$, demonstrating that the data is not missing completely at random. We divided attrition styles into three categories: category 1 (low) included individuals who participated at each of the three waves of data collection $(n=$ 3,434); category 2 (medium) included individuals who participated at Wave 1 and a second 
wave of data collection, either Wave 2 or Wave $3(n=1,330)$; category 3 (high) included individuals who participated exclusively at Wave $1(n=1,754)$. Using these categories, we considered trends in attrition rates as they related to demographic variables, including sex, age, and income.

A series of one-way between-subjects ANOVAs indicated that attrition style categories low, medium, and high differed in relation to each of the demographic variables: sex $(F(2,6473)=13.92, \mathrm{p}<.001)$, age $(F(2,6457=131.69, \mathrm{p}<.001)$, and income $(F(2,5192, \mathrm{p}=$ .016). A Tukey post hoc test (Table 8) revealed that there was a greater trend towards attrition among female, young, and low income participants. However, there was no distinction in sex and income between high and medium attrition categories, or in income between low and medium attrition categories.

Table 8

Sex, age, and income among low, medium and high attrition rates:

Tuckey Post Hoc analyses

\begin{tabular}{|c|c|c|c|c|c|c|}
\hline \multirow[b]{2}{*}{$\begin{array}{l}\text { (I) } \\
\text { Attrition } \\
\text { Category }\end{array}$} & \multirow[b]{2}{*}{$\begin{array}{l}(\mathrm{J}) \\
\text { Attrition } \\
\text { Category }\end{array}$} & \multirow[b]{2}{*}{$\begin{array}{c}\text { Mean } \\
\text { Diff (I-J) }\end{array}$} & \multirow[b]{2}{*}{$\begin{array}{l}\text { Std. } \\
\text { Error }\end{array}$} & \multicolumn{2}{|c|}{$\begin{array}{c}\text { 95\% Confidence } \\
\text { Interval }\end{array}$} & \multirow[b]{2}{*}{ Sig. } \\
\hline & & & & $\begin{array}{l}\text { Lower } \\
\text { Bound }\end{array}$ & $\begin{array}{l}\text { Upper } \\
\text { Bound }\end{array}$ & \\
\hline \multicolumn{7}{|l|}{ Sex. } \\
\hline \multirow[t]{2}{*}{1} & 2 & $-0.05^{*}$ & .016 & -.089 & -.015 & .003 \\
\hline & 3 & $-0.07 *$ & .015 & -.106 & -.038 & $<.001$ \\
\hline \multirow[t]{2}{*}{2} & 1 & $0.05^{*}$ & .016 & .015 & .089 & .003 \\
\hline & 3 & -0.02 & .018 & -.061 & .023 & .529 \\
\hline \multicolumn{7}{|l|}{ Age } \\
\hline \multirow[t]{2}{*}{1} & 2 & $4.60 *$ & .498 & 3.43 & 5.76 & $<.001$ \\
\hline & 3 & 7.10* & .457 & 6.03 & 8.17 & $<.001$ \\
\hline \multirow[t]{2}{*}{2} & 1 & $-4.60 *$ & .498 & -5.76 & -3.43 & $<.001$ \\
\hline & 3 & $2.51^{*}$ & .565 & 1.18 & 3.83 & $<.001$ \\
\hline \multicolumn{7}{|l|}{ Income } \\
\hline \multirow[t]{2}{*}{1} & 2 & 0.03 & .037 & -.053 & .118 & .648 \\
\hline & 3 & $0.10^{*}$ & .034 & .018 & .177 & .011 \\
\hline \multirow[t]{2}{*}{2} & 1 & -0.03 & .037 & -.118 & .053 & .648 \\
\hline & 3 & 0.65 & .042 & -.033 & .164 & .267 \\
\hline
\end{tabular}

$n: 1=3,434 ; 2=1,330 ; 3=1,754$

Sex: Male coded 1; Female coded 2

Unfortunately, Little's MCAR cannot demonstrate whether attrition is Missing at Random (MAR), which suggests that attrition is related to observed variables, or Missing not at Random (MNAR), which suggests that attrition is related to unobserved variables. 
However, Missing at Random is a reasonable assumption in longitudinal studies that include a broad range of variables that may be related to attrition, and it is considered acceptable to then impute the missing scores with scores estimated from the observed variables (Asendorpf, van de Schoot, Denissen, \& Hutteman, 2014). We therefore applied Estimation Maximization, using a single-imputation procedure, to estimate all missing personality variables where the participant had responded to two or more waves of data collection ( $n=$ $4,764)$.

Using the new dataset with imputed scores, we assessed the stability of profile membership within both three and four profile solution by conducting a Latent Profile Transition Analysis, first with an interval of one, and then two, years. When using LPA, profile membership represents a stable set of characteristics. Conversely, in Latent Profile Transition Analysis, individuals may change membership over time. First, latent membership probabilities are estimated at each time point. Following this, transition probabilities are obtained from a multinomial regression of profile membership at the second time point on profile membership at the first time point. The transition probabilities then reflect conditional probabilities for time 2 profiles given time 1 profile membership.

Table 9

Transition probabilities of SFM profiles across 1-year and 2-year intervals

\begin{tabular}{|c|c|c|c|c|c|c|c|c|c|}
\hline & \multirow[b]{2}{*}{$\begin{array}{c}\text { Personality profile in } \\
\text { year } n\end{array}$} & \multicolumn{4}{|c|}{$\begin{array}{c}\text { Personality type in } \\
\text { year } n+1\end{array}$} & \multicolumn{4}{|c|}{$\begin{array}{c}\text { Personality type in } \\
\text { year } n+2\end{array}$} \\
\hline & & $\mathrm{R}$ & 0 & $U$ & & $\mathrm{R}$ & 0 & $U$ & \\
\hline \multirow{4}{*}{$\begin{array}{l}\frac{0}{4} \\
\frac{0}{2} \\
\frac{0}{m} \\
m\end{array}$} & Resilient (R) & 1.00 & $.00^{*}$ & $.00^{*}$ & -- & 1.00 & $.00^{*}$ & .00 & -- \\
\hline & Overcontrol (O) & .00 & .99 & .01 & -- & .00 & 1.00 & $.00^{*}$ & -- \\
\hline & Brittle/Undercontrol (U) & .00 & $.00^{*}$ & 1.00 & -- & $.00^{*}$ & .00 & 1.00 & -- \\
\hline & & $\mathrm{R}$ & $\mathrm{O}$ & $U$ & B & $\mathrm{R}$ & $\mathrm{O}$ & U & B \\
\hline \multirow{4}{*}{$\begin{array}{l}\frac{0}{4} \\
\frac{1}{0} \\
\frac{1}{d} \\
\dot{d}\end{array}$} & Resilient (R) & 1.00 & $.00^{*}$ & $.00^{*}$ & .00 & 1.00 & $.00^{*}$ & $.00^{*}$ & .00 \\
\hline & Overcontrol (0) & $.00^{*}$ & .99 & .00 & .01 & $.00 *$ & 1.00 & $.00 *$ & $.00 *$ \\
\hline & Undercontrol (U) & .02 & $.00^{*}$ & .98 & $.00^{*}$ & $.00 *$ & .01 & .99 & $.00 *$ \\
\hline & Brittle (B) & .00 & $.00^{*}$ & $.00^{*}$ & 1.00 & .00 & $.00^{*}$ & $.00 *$ & 1.00 \\
\hline
\end{tabular}

The entropy for both three and four profile Latent Profile Transition models was high (in both cases .86). Although the current analyses do not provide a measure of the BLRT, the 
BIC favoured the four profile model, which was lower (difference of 842.42) than in the three profile model. However, due to the overpowering of the sample that occurred when conducting the Latent Profile Analyses, we are hesitant to put much faith this measurement. Finally, Latent Transition Probabilities demonstrated that membership within personality types were highly stable for both the three and four profile transition models (Table 9) across one and two year intervals. Membership within both three and four profile undercontrol solutions in this model was more stable than membership within the undercontrol profile identified by Meeus et al. (2011). This may be due to the age of participants within the current sample, as our analyses included exclusively adult participants, while the participants within the Meeus et al. (2011) investigation were adolescents, and therefore more susceptible to fluctuations and changes in personality attributes (Fraley \& Roberts, 2005; Roberts \& Del Vecchio, 2000).

Table 10

Nested model: BIC difference between freely estimated and constrained models

\begin{tabular}{lcc}
\hline & \multicolumn{2}{c}{ Interval } \\
\cline { 2 - 3 } & 1 year & 2 year \\
\hline Three Profile & 111.81 & 64.64 \\
Four Profile & 160.69 & 114.42 \\
\hline$n=4,764$ & \\
$*$ difference represents BIC score of freely- \\
estimated model minus BIC of constrained model
\end{tabular}

As a final step to assess profile stability, we tested measurement invariance of the configuration of personality traits within each profile over time. After testing the freely estimated model (discussed above), we constrained item-response probabilities within each profile to be equal across assessment periods. Because these models are statistically nested, we can then use the difference between each model's BIC scores to establish whether measurement invariance holds across times (Collins \& Lanza, 2009). In essence, this test allows us to demonstrate whether the meaning of the latent profiles changes over assessment periods. The BIC differences between freely-estimated and constrained Latent Profile Transitions are listed in Table 10. Across both one and two-year intervals, the constrained models demonstrated smaller BIC scores, thereby allowing us to confirm that measurement invariance holds across times. In both constrained and unconstrained models, empty cells were automatically constrained to zero by MPLUS software to avoid singularity. 
These cells represented instances where there was no transition between profiles across intervals. As such, there was no variation within these cells, and regression coefficients could not be determined.

Although both three and four profile solutions are robust, stable, and generally interpretable in terms of the theoretical literature, the current findings supported by the theoretical literature promote an understanding where the four profile solution is regarded as the superior model. Consistent with the theoretical basis of a four profile model as reflecting opposing trends in control strategies (over- and under-control) and in resilience (high- and low-resilience), each pair of profiles in the four profile solution was associated with contrasting outcomes in either inhibitory trends (control) and in adaptability (resilience). Moreover, as expected, overcontrol and undercontrol profiles within the four profile model showed no distinct relationship with the maladaptive outcomes, suggesting that neither control strategy within this sample represented inherently adaptive or maladaptive tendencies. By contrast, the three profile solution appears to combine a core maladapted set of participants (brittle) with individuals reflecting low-inhibitory qualities, suggestive of Block's (1971) early conceptualization of low-functioning undercontrollers. This solution does not, however, identify a relationship between maladjustment and highinhibitory qualities, which would be expected using Block's (1971) conceptualization of lowfunctioning overcontrollers. The current three profile empirical solution we obtained therefore did not fully satisfy theoretical expectations from the literature.

\section{Discussion}

The present research compared three and four profile personality solutions within a large representative sample of adult New Zealanders. Due to the theoretical overlap between ego-resilient and ego-control self-regulatory styles (Block \& Block, 1980), and the proposed curvilinear relationship between polar extremes of control and low-resilience (Asendorpf \& van Aken, 1999; Block, 1971; Robins et al., 1996), the distinction between a three and four profile solution is a complex question to investigate. The present investigation therefore used both Latent Profile and Latent Profile Trajectory Analyses to address this question.

To our knowledge, only one previous investigation (Meeus et al., 2011) employed these techniques to study personality profiles. This previous investigation converged on a three profile solution, where additional profiles represented proportions of the overall sample 
that were too small for meaningful transition analysis (Meeus et al., 2011), precluding the option of a direct comparison of the three and four profile solutions. The current analysis therefore provides a novel opportunity to consider and compare the interpretability of these structures in greater detail. Based on the structural configurations and predictive ability of these profiles, and guided by Block and Block's (1980) self-regulatory theory of personality functioning, we propose that the four profile model of personality, which incorporates resilient, brittle, overcontrol and undercontrol prototypical personality patterns, provides an interpretable and informative distinction over and above the frequently identified three profile model.

\section{Fit and Stability of Three and Four Profile Solutions}

In the current analyses, the relative entropy across both potential models (three and four profile solutions) was medium, which suggested an interpretable degree of overlap between profile structures, and the average class membership probabilities were robust, indicating that participants assigned to different profile structures had a reasonably strong convergence with that profile. Each profile represented a reasonably large portion of the sample population, suggesting that profiles were not indicative of small non-normal fluctuations in the data. Moreover, measurement invariance held across intervals for each solution, and each profile was longitudinally stable, with transition probabilities demonstrating near-perfect consistency in profile assignment. This latter result differs from Meeus et al. (2011), who found longitudinal stability to be low in the undercontrol profile. This could be a consequence of more precise profiles being developed from a larger sample (close to four times bigger in the current longitudinal analyses) or perhaps the specific demographic characteristics of participants. More specifically, personality stability tends to increase with age (Fraley \& Roberts, 2005), and participants studied by Meeus et al. (2011) ranged from early-to-late adolescence, whereas the current sample was comprised exclusively of adults $(M=47.91, S D=15.73)$.

\section{Structure and Interpretability}

Resilient and brittle profiles are conceptualised as corresponding to two opposing poles of the ego-resilience dimension. In previous models, the resilient profile is typically characterised by high scores on Extraversion, Agreeableness, Conscientiousness, and Openness, as well as low Neuroticism (high Emotional Stability). This pattern is replicated in 
the current analyses; however, we also identified a profile characterised entirely by low, or undesirable, trait scores, which we suggest represents the brittle profile. Consistently, overcontrol and undercontrol profiles are conceptualised as corresponding to two opposing poles on the ego-control dimension. Despite inconsistencies in previous studies, researchers largely agree that undercontrol groups are represented by high scores in Extraversion and Openness, while overcontrol groups tend to score low on these traits (Ekehammar \& Akrami, 2003; Gramzow et al., 2004; Robins et al., 1996). This pattern is replicated in the current analyses, with an additional distinction between profiles regarding the trait Honesty/humility (reflecting tendencies towards restraint/inhibition), which presents as high in overcontrollers and low in undercontrollers. The overall structure of personality traits that distinguish between over and undercontrol profiles are therefore plausibly interpretable according to the self-regulatory literature.

\section{Predictive Ability}

As expected, higher probabilities of being classified into either the resilient and brittle profiles predicted desirable vs. undesirable outcomes, respectively, in both intra- and interpersonal domains. Greater probability of membership in the resilient profile was positively associated with wellbeing and good perceived quality of interethnic relations, and was negatively associated with Social Dominance Orientation. Greater probability of membership in the brittle profile was associated with the opposite relationship with all three outcomes. Consistently, Block and Block's (1980) ego-regulation theory supports the existence of such self-regulatory patterns that vary from high to low functioning, interpersonal competence vs. incompetence, and adaptive vs. maladaptive responses to stress and change.

Also as expected, probability of membership in both undercontrol and overcontrol profiles predicted Openness to Change and Self-Enhancement; higher levels of overcontrol correlated negatively, and higher levels of undercontrol correlated positively, with these higher-order values. Openness to Change and Self-Enhancement values are both characterised by a focus on the individual (Schwartz, 2011). Moreover, the combination of both Self-Enhancement and Openness to Change has been associated with an emphasis on independent thought, as well as a tendency to engage in risky behaviours and to pursue success in an exaggerated manner (Licht, 2007; Sarracino, Presaghi, Degni, \& Innamorati, 
2011).The uninhibited qualities of undercontrollers, in combination with their sensationseeking and pleasure-focused tendencies, therefore make them prime candidates for this combination, while the more cautious, inhibited and restrained overcontrollers should show the reverse trend.

Similarly, mean-level differences demonstrated clear trends relating to adaptive and inhibitory outcomes. Resilient and brittle profiles demonstrated the most well- and maladapted outcomes, whereas over- and undercontrollers demonstrated the highest and lowest inhibitory outcomes, respectively. The current findings are therefore eminently interpretable in terms of Block and Block's (1980) theory of ego-resilience and ego-control, and support our identification of the current profiles as representing high vs. low levels of ego-resilience and ego-control. Moreover, the combination of continuous and categorical methodologies used here lends credence to the theoretical interpretation of these solutions as reflecting degrees of prototypicality. Specifically, each type represents distinct groupings of characteristics, whereas variance in the degree of convergence with each type results in interpretable differences between individuals.

\section{Comparisons across Three and Four Profile Solutions}

Notably, participant distribution, trait configurations, and predictive ability of both the resilient and overcontrol profiles were nearly identical across both three and four profile solutions. Conversely, the three solution undercontrol profile incorporates trends, participants, and outcomes from both four solution undercontrol and brittle profiles. This finding could be interpreted as relating to the curvilinear relationship between lowresilience and control dimensions; however this interpretation is highly inconsistent with the finding that the overcontrol profile did not demonstrate any such curvilinear trends. Moreover, given that the current prototypical configurations represent group-level trends around profiles (rather than converging on the most extreme cases of high/low control), we suggest that it is more informative to consider the core profiles as converging on Block and Block's (1980) four desirable/non-desirable, and inhibited/non-inhibited constructs. The predictive ability of the four profile model was consistent with this theoretical foundation, and allowed clearer, more specialised predictions, as compared to the three profile model. Specifically, outcomes relating to the four profile model varied as expected along either 
adjustment or inhibition characteristics, whereas outcome relating to the three profile model varied along 1) adjustment 2) inhibition 3) adjustment and inhibition.

\section{Limitations and Future Research}

Major limitations in the current research includes issues with multi-colinearity, which restricts the ability to consider the models in terms of variance explained when considering placement of participants on all profiles simultaneously. An additional limitation concerns the use of personality markers rather than longer, more detailed, trait measurements. Although the Mini-IPIP6 is demonstrated to be an effective stand-in for more detailed traitmeasures (Sibley, Harding, Perry, Asbrock, \& Duckitt, 2010), using short markers does come at the expense of trait complexity, and could conceivably reduce the intricacy and predictive power of resultant profiles. Future research should consider more detailed personality measures in composite profiles to establish whether the present results are replicated.

The validity of using a 6 rather than 5 factor model of personality in constructing the profiles should also be assessed by including both models in a comparative analysis of model-fit and predictive ability. Although outside the scope of the current paper, this will allow researchers to assess whether the inclusion of the additional trait serves to enhance or reduce the interpretability of the resultant structures, and will permit clearer points of comparison between the current work and previous investigations.

The current findings provide support for the presence of stable configurations of personality working within the individual as a coordinated regulatory system. These profiles are most interpretable in terms of the four profile model originally suggested within Block and Block's (1980) extensive longitudinal investigation of ego-constructs. Additionally, the four profile model identified here provides a better foundation for a substantive theoretical interpretation of the personality structures, which in turn enhances the ability of these profiles to provide a truly complementary approach to the traditional variable-centred approach. 
STUDY 2. Validation of the Four Profile Configuration of Personality Types within the FiveFactor Model

\begin{abstract}
Prototypes of personality traits using the Five-Factor Model of personality were developed using Latent Profile Analysis. Trait configurations and predictive outcomes suggested the appropriateness of a four profile solution over the commonly identified three profile solution. However, comparisons of model fit and predictive ability with previous investigations suggest that the profiles presently developed are less precise than those using the alternative Six-Factor Model of personality. Accordingly, the results indicate that the additional trait within the Six-Factor Model provides critical information in profile development. The authors argue that, regardless of whether the Five-Factor or Six-Factor Model of personality is used, the four profile solution more comprehensively reflects a framework for describing coherent and meaningful profiles.
\end{abstract}




\section{Introduction}

A growing body of research in personality psychology has focused on personality types (e.g., Asendorpf et al., 2001). In contrast to the 'variable-centred' approach, which describes differences across individuals with particular variables as the primary units of measurement, research on personality types examines configurations of traits within the individual. This latter approach accounts for the whole person engaged with their environment, and thus provides a more coherent explanation of personality functioning (Donnellan \& Robins, 2010). Moreover, by identifying subsets of individuals with similar configurations of traits, researchers may be able to gain greater insight into the underlying mechanisms that produce both within-person variation and between-person differences across the observed dimensions.

Block and Block's (1980) model of ego-control and ego-resiliency is often advanced as a theoretical framework for understanding and interpreting personality types (Asendorpf et al., 2001). Individual differences in ego-control reflect the tendency to control or inhibit impulses and desires, whereas varying levels of ego-resilience are grounded in the flexibility of responding styles, and reflect levels of well-adjusted functioning. These self-regulatory constructs generate emotional and behavioural patterns, which may be expressed within configurations of personality dimensions (Gramzow et al., 2004).

Consistent with Block and Block's (1980) theoretical framework, a recent investigation (Isler, Liu, Sibley \& Fletcher, 2016) identified a four profile solution of high/low resilient/control personality trait configurations within a large, longitudinal national probability sample. These profiles demonstrated strong longitudinal stability, and a pattern of relationships consistent with Block and Block's (1980) ego-constructs. Specifically, high/low resilient profiles differed primarily on adaptive vs. maladaptive outcomes whereas high/low control profiles differed on interpersonal motivational values related to inhibition, pleasure focus and pursuit of new experiences.

In contrast to this study (Isler et al., 2016), much prior research has converged on a three profile solution of traits (e.g., Asendorpf et al., 2001; Meeus et al., 2011), leading many to conclude that the three major 'replicable' personality prototypes are undercontrol, resilient, and overcontrol types. Advocates of this model suggest that the three profile structures converge on a curvilinear relationship between low-adjustment and both extremes of the control dimension (Asendorpf et al., 2001). 
However, the three type solution has not always been found (e.g. Rammstedt, Riemann, Angleitner, \& Borkenau, 2004), and even when three profiles are reported, the configurations are often inconsistent across studies (see Herzberg \& Roth, 2006). Donnellan and Robins (2010) suggested that the lack of replicability may be a product, in part, of different analytic procedures and sampling biases, including low sample sizes and nonrepresentative sampling methods. Moreover, the three profile model may overlook important theoretical distinctions between profiles outlined within a four profile model (see Gramzow et al., 2004). For example, the four profile solution reported by Isler et al. (2016) is elegantly consistent with Block and Blocks' (1980) model of self-regulatory processes, and underlines the adaptive qualities within both control tendencies.

Unfortunately, comparisons between the four profile solution identified by Isler et al. (2016) and prior research are complicated by the number of traits considered. Specifically, most previous investigations utilise the Five-Factor Model of personality, whereas Isler et al. (2016) included Honesty-humility as an additional sixth trait in profile development. As suggested by Asendorpf et al. (2001), the number and structure of different personality configurations may, in part, be a function of the number and nature of the traits assessed. Consequently, it is necessary to directly assess the theoretical interpretability and predictive ability of the four profile solution within the commonly tested Five-Factor Model to promote cross-study comparisons. Moreover, the extent to which the Five-Factor Model provides a sufficient foundation for profile development can be assessed by comparing the resultant outcomes to those developed using six-traits (Isler et al., 2016). To accomplish this, the current investigation considered the theoretical and empirical interpretability of the three and four profile solutions of personality profiles within the Five-Factor Model, using the same procedures, and examining the same sample, as initially tested by Isler et al. (2016). Analyses were conducted to examine model fit, interpretability, and predictive ability, of both three and four profile solutions.

The following three predictions were advanced:

1. A four profile solution, interpretable in terms of Block and Block's (1980) selfregulatory constructs, will emerge when using the Five-Factor Model of personality; 
2. A four profile solution will provide superior predictions, than a three profile model, in relevant outcome variables linked to a) adaptive/maladaptive functioning and b) inhibited/unconstrained motivational styles;

3. The loss of information represented within the Five-Factor, as opposed to the SixFactor Model, will correspond to a reduction in the interpretability and predictive strength of personality profiles.

\section{Method}

\section{Sample}

We analysed responses from 6,518 individuals who participated in Wave 1 of the New Zealand Values and Attitudes Survey (NZAVS). Participants were randomly sampled from the New Zealand electoral role. Females represented the majority of participants (59.3\%) and mean age of the respondents was $47.91(S D=15.73)$.

\section{Measures $^{2}$}

Personality: Mini-IPIP. Participants completed the 5 factor 20-item Mini-IPIP (Donnellan et al., 2006). Each personality item consists of a phrase evaluating a trait-relevant behaviour (e.g., 'I have a good word for everyone'), and is measured on a 7-point Likert scale. An exploratory factor analysis (Appendix A) demonstrated that items loaded strongly onto the appropriate factors, while internal consistency reliability values for each factor were high, given the small number of items: Openness $(a=.67)$, Agreeableness $(a=.67)$, Extraversion $(a=.71)$, Conscientiousness $(a=.65)$, and Emotional Stability $(a=.64)$.

Outcome variables. For detailed information regarding item development, refer to Sibley (2009). All items were measured on a 7 point Likert scale, excluding 'Satisfaction with Life' items, which were measured on a 10 point Likert scale, and Schwartz's (1992) values, which were measured on a 9 point Likert scale.

Subjective wellbeing. Nine items targeting subjective wellbeing were measured (details below). Internal consistency for the scale was high $(a=.84)$. Z-scores were calculated and averaged to provide a total score.

a. Self-Esteem: three items from Rosenberg (1965).

\footnotetext{
${ }^{2}$ For scale items and instructions, view Appendix B
} 
b. Personal Wellbeing: four items from Cummins et al. (2003).

c. Satisfaction with Life: two items from Diener et al. (1985).

Perceived quality of interethnic relations. Single-item scales measuring perceptions of realistic (Bobo, 1998) and symbolic (Stephan et al., 2002) threat to New Zealanders in general were repeated four times in reference to different ethnic groups (Maori, NZ Europeans, Pacific Islanders, Asians). Internal consistency was high for both realistic $(a=.83)$ and symbolic $(a=.85)$ threat, and items were averaged to produce total scores. Three additional single-item measures were then included (details below). Given the few items, internal reliability for the 5 item scale was good $(a=.66)$. Items were averaged to produce a total score.

a. Racial essentialism: No et al. (2008).

b. Intergroup anxiety: adapted by Sibley and Barlow (2009; cited in Sibley, 2009) from Stephan and Stephan (1985).

c. Race-based rejection sensitivity: adapted by Sibley and Barlow (2009; cited in Sibley, 2009) from Shelton and Richeson (2005).

Social dominance orientation. Participants completed six items from the Social Dominance Orientation scale (Sidanius \& Pratto, 1999). Internal consistency reliability for this scale $(a=.69)$ was good.

Self-enhancement and openness to change values. Participants completed a 12 -item measure of Schwartz's (1992) values, adapted by Stern et al. (1998). For the current analyses, we only consider the Openness to Change and the Self-Enhancement items. Given the small number of items, internal consistency reliability was high for both Openness to Change $(a=.73)$, and Self Enhancement ( $a=.61)$ values.

\section{Procedure}

We developed and assessed three and four profile solutions using Latent Profile Analyses of participants' responses to the Five-Factor Model trait measures using MPLUS software (Muthén \& Muthén, 2012). We compared the two different solutions by considering fit indices and examining trait configurations from a theoretical framework. Following this, we calculated the proportions of participants represented by each profile, and analysed demographic information by regressing probability of class membership for each personality 
profile on age, sex and income simultaneously. After controlling for demographic information, we regressed each of the three and four solution profiles on subjective wellbeing, perceived quality of interethnic relations, Social Dominance Orientation, and SelfEnhancement and Openness to Change values. Finally, we compared mean-level differences relating to each of the five outcome variables using a series of ANOVAs.

\section{Results}

\section{Model Fit}

Latent Profile Analysis provides multiple tools to consider and compare model fit and interpretability. Bayesian Information Criterion (BIC; Schwarz, 1978) is used to compare across two different models, with smaller values indicating better model fit. The Bootstrap Likelihood Ratio Test (BLRT; McLachlan \& Peel, 2000) produces a $p$ value to represent the likelihood that the solution being investigated is superior to a hypothetical solution with one less class. These two fit statistics continued to demonstrate a substantial improvement of fit upon the inclusion of additional profiles, even when profile memberships were no longer large enough to provide informative distinctions between groups, indicating an overpowering of the sample (see also Marsh et al., 2009).

Entropy and average probability of class membership scores are used to demonstrate the amount of within-profile potential for misclassification. Both scores are measured between $[0,1]$, with higher values indicating lower misclassification and more discrete personality types. Although there is no clear cut-off point for either score, Clark and Muthèn (2009) suggest an entropy of $0.8,0.6$, and 0.4 as representing high, medium, and low entropy, respectively. Additionally, the generally recognised rule of thumb for acceptable profile classification is when the average posterior class membership probability is 0.70 or greater (Nagin, 2005).

Table 11 presents fit statistics for solutions ranging from $3-4$ classes. In each solution, the entropy was low to medium, demonstrating some overlap between classes. The average posterior class membership probabilities suggested that, despite the overlap between classes, the mean probability of type membership (or convergence with the prototypical profile) was acceptable, at about $70 \%$. Both three and four profile solutions demonstrated moderately lower fit as compared to the solutions developed using the Six-Factor Model 
(Isler et al., 2016), suggesting that the loss of information represented here by the exclusion of the sixth trait corresponded to less discrete profile solutions.

Table 11

Five-Factor Model (FFM) personality profiles:

Fit statistics for 3 and 4 profile solutions

\begin{tabular}{llcccc}
\hline & & \multicolumn{4}{c}{ Average Probability of Class Membership } \\
\cline { 3 - 6 } & Entropy & Profile 1 & Profile 2 & Profile 3 & Profile 4 \\
\hline 3 Profile Solution & .43 & .73 & .70 & .75 & - \\
4 Profile Solution & .50 & .68 & .69 & .72 & .72 \\
\hline$n=6,518$ & & & & &
\end{tabular}

We then assessed whether profiles represented adequately interpretable proportions of the sample (greater than 5 percent of the total sample). As demonstrated in Table 12, each profile represented over 10 percent of the total sample in both solutions, suggesting that solutions were not indicative of non-normal fluctuations within the data.

Table 12

Participant distribution in FFM personality profiles:

Percent of total sample

\begin{tabular}{lcccc}
\hline & One & Two & Three & Four \\
\hline 3 Profile Solution & 27.2 & 17.9 & 55.0 & -- \\
4 Profile Solution & 23.3 & 12.4 & 50.2 & 14.1 \\
\hline$n=6,518$ & & & &
\end{tabular}

\section{Theoretical Interpretability}

Prototypical trait configurations of the three and four profile solutions were developed by calculating mean trait scores for all participants ultimately assigned to each profile. Figures $2 \mathrm{a}$ and $2 \mathrm{~b}$ show the resultant profile structures. The four profile model was consistent with Block and Block's (1980) control/resilience dimensions. Specifically, one profile was characterised by high (desirable) mean values on each personality trait (suggesting high resilience), and another was characterised by the opposite pattern (suggesting low resilience). Additionally, a low-inhibitory (undercontrol) profile was marked by high Extraversion and Openness, with low levels of Conscientiousness, whereas a high-inhibitory (overcontrol) profile demonstrated the opposite trend.

The current three profile solution was comprised of high/low resilient and high-control profiles equivalent to those identified within the four profile solution. These results offer mixed interpretability with previous investigations. Specifically, although this resilient profile is commonly identified within three profile solutions (e.g., Asendorpf et al., 2001; Dubas et 
al., 2002; Meeus et al., 2011), the overcontrol solution mirrors results from some previous literature (Dubas et al. 2002; Meeus et al., 2011) with no observable counterpart in a number of other studies (Asendorpf et al., 2001; Berry et al., 2007). Finally, the pattern of traits reflected in the low resilient (brittle) profile is inconsistently interpreted in previous studies, being variously categorised as undercontrol (Dubas et al., 2002) overcontrol (Berry et al., 2007) and undesirable (Barbaranelli, 2002).

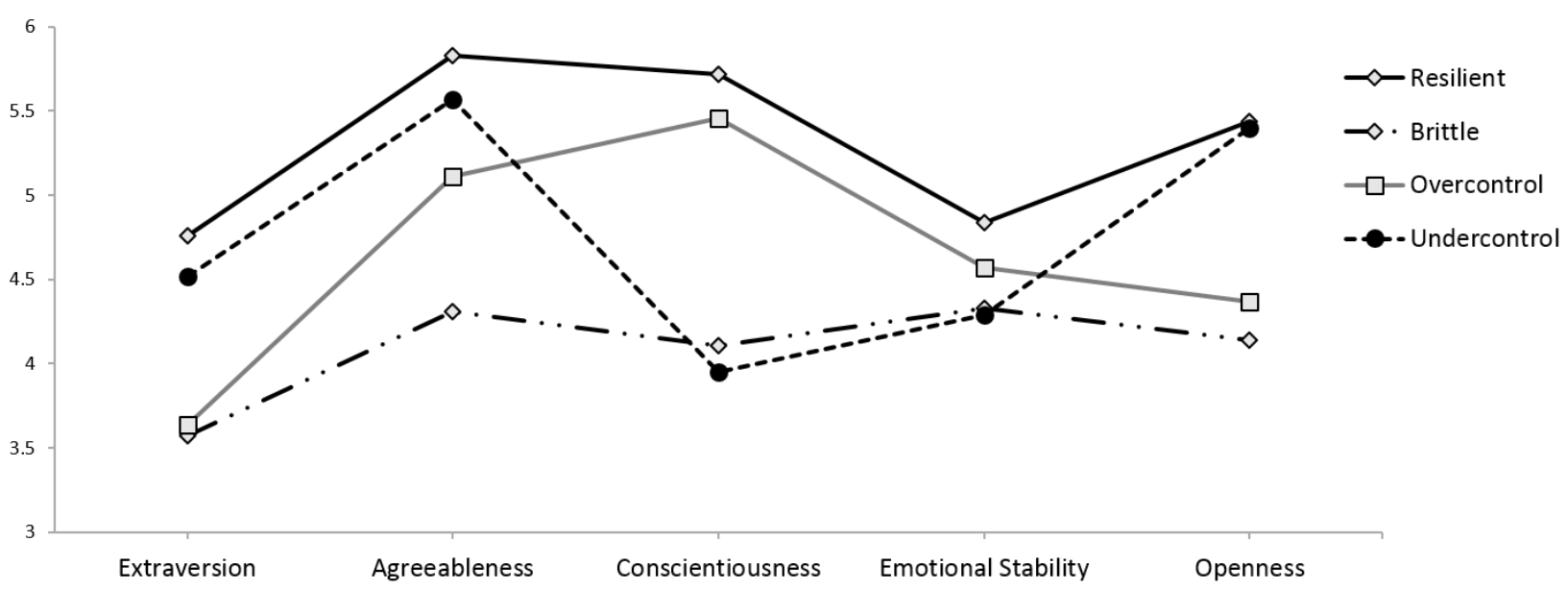

Figure 2a: Four Profile solution of personality traits (Five-Factor Model)

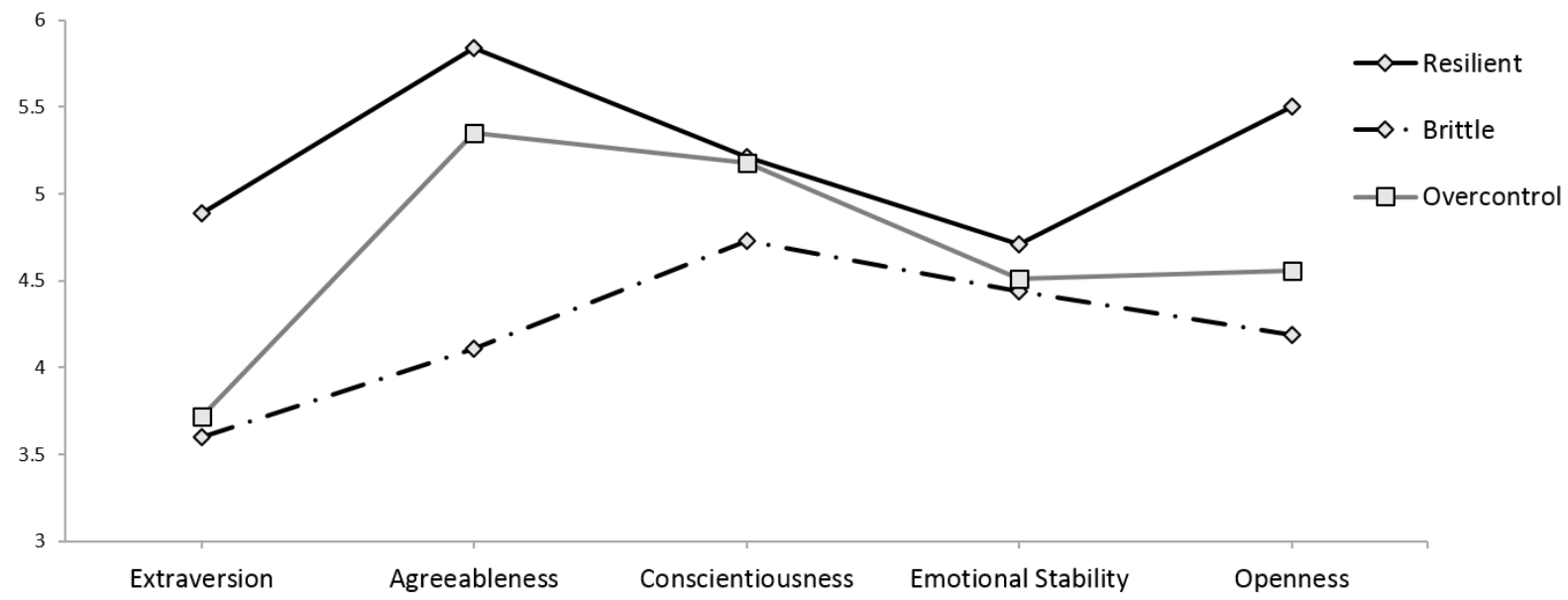

Figure 2b: Three Profile solution of personality traits (Five-Factor Model)

\section{Predictive Ability}

Before investigating the relationship between personality profiles and outcome variables, we assessed the relationship between personality profiles and demographic information. As presented in Table 13, sex, age, and income showed small, but significant, relationships with 
personality profiles. In both three and four profile solutions, resilient profiles were associated with being female and high income, whereas brittle profiles were associated with being male and low income. Age was positively related to overcontrol profiles, and in the four profile model was negative related to undercontrol. We controlled for these variables when regressing outcome variables on personality profiles.

Table 13

FFM personality profile membership regressed on demographic variables: Standardised beta weights

Three profile solution

\begin{tabular}{|c|c|c|c|c|c|}
\hline & & Resilient & Brittle & Overcontrol & \\
\hline Sex & $\beta$ & $.13^{* *}$ & $-.25 * *$ & $.10 * *$ & --- \\
\hline Age & $\beta$ & $-.11 * *$ & .01 & $.12^{* *}$ & --- \\
\hline Income & $\beta$ & $.08^{* *}$ & $-.08 * *$ & -.02 & --- \\
\hline \multicolumn{6}{|c|}{ Four profile solution } \\
\hline & & Resilient & Brittle & Overcontrol & Undercontrol \\
\hline Sex & $\beta$ & $.13^{* *}$ & $-.20 * *$ & .02 & -.01 \\
\hline Age & $\beta$ & -.02 & .00 & $.15^{* *}$ & $-.17 * *$ \\
\hline Income & $\beta$ & $.09 * *$ & $-.09 * *$ & -.02 & .02 \\
\hline
\end{tabular}

$n=6,518$

** Significant at $<.001$

Sex: Male coded 1; Female coded 2

Predictors entered simultaneously

Adaptive and maladaptive tendencies (Wellbeing, perceived quality of interethnic relations, and Social Dominance Orientation), as well as inhibitory and non-inhibitory tendencies (Openness to Change, and Self Enhancement values) were then regressed individually onto each profile within both three and four profile solutions. Standardised regression coefficients for each profile/DV relationship are listed in Table 14.

Outcomes associated with the four profile solution were generally consistent with the theoretical foundation of personality profiles (Block \& Block, 1980). The resilient profile was positively associated with wellbeing, and perceived quality of interethnic relations, whereas the brittle profile was negatively associated with these outcomes. Also as expected, the reverse pattern was found between Social Dominance Orientation and high/low resilience. However, this relationship was weak, and was matched in strength to the marginal trend found between high/low control and Social Dominance Orientation. As expected, the overcontrol profile was negatively associated with Openness to Change, however the undercontrol profile shared only a marginal trend with this variable. Finally, contrary to what would be expected of profiles that represent differences in ego-control, the overcontrol profile shared no relationship with Self-Enhancement values, whereas the 
undercontrol profile unexpectedly demonstrated a marginal negative trend with this outcome.

Table 14

Beta weight of FFM profile membership regressed on outcome variables

\begin{tabular}{|c|c|c|c|c|c|c|}
\hline & Profile & $\begin{array}{l}\text { Personal } \\
\text { Wellbeing }\end{array}$ & $\begin{array}{c}\text { Perceived } \\
\text { Interethnic } \\
\text { Relations }\end{array}$ & $\begin{array}{c}\text { Social } \\
\text { Dominance } \\
\text { Orientation }\end{array}$ & $\begin{array}{c}\text { Openness to } \\
\text { Change } \\
\text { Values }\end{array}$ & $\begin{array}{c}\text { Self } \\
\text { Enhancement } \\
\text { Values }\end{array}$ \\
\hline \multirow{3}{*}{$\begin{array}{l}\frac{0}{6} \\
\frac{0}{0} \\
\frac{0}{m} \\
m\end{array}$} & Resilient & $.21 * *$ & $.26^{* *}$ & $-.16 * *$ & $.30 * *$ & $.08^{* *}$ \\
\hline & Brittle & $-.14 * *$ & $-.24 * *$ & $.20 * *$ & $-.17 * *$ & $-.03 *$ \\
\hline & Overcontrol & $-.11^{* *}$ & $-.07 * *$ & -.01 & $-.18^{* *}$ & $-.07 * *$ \\
\hline \multirow{4}{*}{$\frac{\frac{0}{4}}{\frac{0}{0}}$} & Resilient & $.26^{* *}$ & $.22 * *$ & $-.12 * *$ & $.25^{* *}$ & $.11^{* *}$ \\
\hline & Brittle & $-.19 * *$ & $-.20 * *$ & $.13^{* *}$ & $-.14 * *$ & $-.05 * *$ \\
\hline & Overcontrol & $-.03 *$ & $-.14^{* *}$ & $.10 * *$ & $-.22 * *$ & -.02 \\
\hline & Undercontrol & $-.10 * *$ & $.10 * *$ & $-.11 * *$ & $.11 * *$ & $-.07 * *$ \\
\hline
\end{tabular}

$n=6,518$

* Significant at $<.01 ; *$ Significant at $<.001$

All regressions are conducted after controlling for demographic variables

Predictors entered individually

Outcomes relating to the resilient, brittle, and overcontrol profiles within the three profile model matched the equivalent patterns identified within the four profile model. This adds credence to the interpretation of this low-scoring profile as being representative of a low-resilient maladaptive response system as opposed to a low-control personality characterised by greater expression of impulses and feelings.

A series of one-way between-subjects ANOVAs were conducted comparing profiles within both three and four profile solutions to examine mean-level differences relating to each of the five outcome variables. Due to heterogeneous variances between profiles, Welch's robust test of equality of means was used to compare means across profiles. Results (Tables 15 and 16) confirmed that there was a significant pattern of means in both three and four profile solutions.

Tukey post-hoc analyses were performed to investigate the specific characteristics of these differences. Patterns associated with the four profile model (Table 15) were consistent with both over and under-control profiles as reflecting contrasting domains on one continuum, and both resilient brittle profiles on another. However, the outcomes were less clear-cut as compared to those developed using the Six-Factor Model of personality (Isler et al., 2016). 
More specifically, consistent with our previous interpretations of the four profile prototypes, the resilient profile demonstrated the highest, and the brittle profile the lowest, levels of adjustment on all the three adjustment outcomes (Personal Wellbeing, Perceived Interethnic Relations, and Social Dominance Orientation). However, the undercontrol profiles also demonstrated lower Personal Wellbeing but higher interpersonal adjustment (high Perceived Interethnic Relations and low Social Dominance Orientation) as compared to the overcontrol profile.

As expected, the overcontrol profile endorsed lower Openness to Change values as compared to the undercontrol profile. However, contrary to theoretical expectations, the level of high versus low inhibitory outcomes were best predicted by resilient and brittle profiles rather than either over or under-control profile. Specifically, endorsement of Openness to Change values was highest among resilient participants, and lowest among brittle participants. Additionally, Self-Enhancement values were highest among resilient participants, with no significant differences between brittle, overcontrol, and undercontrol participants. These findings are inconsistent with the theoretical foundation of egoconstructs, and suggest that the current four profile solution does not provide an unambiguous differentiation between control and resilience dimensions as predicted by Block and Block's (1980) theoretical foundation.

Table 15

FFM four profile solution:

One-way ANOVAs predicting mean-level differences in outcome variables

\begin{tabular}{lccccccccccc}
\hline \multirow{2}{*}{ Dependent variables } & \multicolumn{2}{c}{ Resilient } & \multicolumn{2}{c}{ Brittle } & \multicolumn{2}{c}{ Overcontrol } & \multicolumn{2}{c}{ Undercontrol } & \multirow{2}{*}{ Welch's $F$} \\
\cline { 2 - 9 } & $M$ & $S D$ & $M$ & $S D$ & $M$ & $S D$ & $M$ & $S D$ & \\
\hline Personal Wellbeing & .26 & .60 & $-.24^{\mathrm{a}}$ & .69 & -.01 & .64 & $-.17^{\mathrm{a}}$ & .76 & $142.74^{*}$ \\
Social dominance orientation & $2.39^{\mathrm{b}}$ & .96 & 2.89 & .98 & 2.68 & .94 & $2.39^{\mathrm{b}}$ & .97 & $69.11^{*}$ \\
Percieved Interethnic Relations & 5.10 & .83 & 4.57 & .84 & 4.76 & .78 & 4.97 & .83 & $96.07^{*}$ \\
Self-Enhancement Values & 3.89 & 1.42 & $3.58^{\mathrm{cd}}$ & 1.50 & $3.71^{\mathrm{de}}$ & 1.34 & $3.58^{\mathrm{ce}}$ & 1.38 & $13.06^{*}$ \\
Openness to Change Values & 5.27 & 1.19 & 4.41 & 1.45 & 4.55 & 1.31 & 5.11 & 1.19 & $157.12^{*}$ \\
\hline
\end{tabular}

Note. $M=$ Mean; $S D=$ Standard Deviation. ${ }^{*} p<.001$

Means with similar superscripts did not differ significantly $(p>.05)$.

Comparably, mean-level differences within the three profile solution (Table 16) were consistent with those represented in the four profile solution. Resilient and brittle profiles 
mirrored the adjusted and maladjusted trends associated with resilient and brittle profiles respectively for the four profile solution. Consistently, the overcontrol profile demonstrated high-inhibitory tendencies reflected by low Self-Enhancement and Openness to Change values (however, the brittle profile demonstrated the lowest scores in Openness to Change values). These findings lend support to our previous suggestion that the three profile brittle solution reflects primarily maladaptive, rather than low-inhibitory, tendencies.

Taken as a whole, these findings suggest that the four profile model is more consistent with Block and Block's (1980) theoretical foundation of personality profiles as compared to a three profile model. Mean-level differences suggest that resilient and brittle profiles reflect adaptive and maladaptive tendencies respectively. Additionally, trait patterns, as well as mean-level differences, suggest that the over and under-control profiles reflect two contrasting domains of self-regulatory tendencies. However, outcomes associated with SelfEnhancement values were less theoretically interpretable. Unlike the previous investigation using the Six-Factor Model (Isler et al., 2016) resilient participants endorsed SelfEnhancement values the most, whereas Self-Enhancement values did not differ between the remaining three profiles. This suggests that the loss of information when Honestyhumility is not included as sixth trait reduces the meaningful differences between personality profiles.

Table 16

FFM three profile solution:

One-way ANOVAs predicting mean-level differences in outcome variables

\begin{tabular}{|c|c|c|c|c|c|c|c|}
\hline \multirow{2}{*}{ Dependent variables } & \multicolumn{2}{|c|}{ Resilient } & \multicolumn{2}{|c|}{ Brittle } & \multicolumn{2}{|c|}{ Overcontrol } & \multirow{2}{*}{ Welch's $F$} \\
\hline & $M$ & $S D$ & $M$ & $S D$ & $M$ & $S D$ & \\
\hline Personal Wellbeing & .19 & .64 & -.16 & .69 & -.04 & .66 & $113.28^{*}$ \\
\hline Social dominance orientation & 2.37 & .97 & 2.98 & 1.00 & 2.59 & .92 & $129.71 *$ \\
\hline Percieved Interethnic Relations & 5.09 & .84 & 4.57 & .82 & 4.81 & .79 & $141.65^{*}$ \\
\hline Self-Enhancement Values & 3.85 & 1.41 & 3.71 & 1.46 & 3.65 & 1.35 & $12.13^{*}$ \\
\hline Openness to Change Values & 5.31 & 1.17 & 4.48 & 1.41 & 4.62 & 1.30 & $231.68^{*}$ \\
\hline
\end{tabular}

Note. $M=$ Mean; $S D=$ Standard Deviation. ${ }^{*} p<.001$.

All means differed significantly $(p<.01)$. 


\section{Discussion}

Prior research by Isler et al. (2016) suggested that a four profile solution of traits is superior to the standard three profile model on both theoretical and empirical grounds. However, direct comparisons across these two competing solutions is confounded by the number of personality traits assessed when developing prototypes. Specifically, the four profile solution developed by Isler et al. (2016) incorporated six traits, whereas research identifying a three profile solution have typically used five traits (Asendorpf et al., 2001; Meeus et al., 2011). To deal with this problem, the present study re-analysed the original data set from Isler et al. (2016) by conducting a Latent Profile Analysis using five traits (the traditional Five-Factor Model).

The results can be readily summarised. Consistent with prior research, the three profile model demonstrated an acceptable fit with five traits. However, in line with our first two predictions, 1) a four profile model was identified, and 2) demonstrated a more interpretable fit and clearer predictive ability in terms of Block and Block's (1980) model of self-regulatory constructs. Finally, consistent with our third prediction, 3) the four profile solutions identified in the current study using the Five-Factor Model of personality resulted in less coherent interpretability and predictive strength, as compared to the four profile solution identified by Isler et al. (2016) using the more detailed Six-Factor Model of personality.

\section{Profile Interpretability}

Three equivalent configurations, reflecting tendencies towards high/low resilience and high control, were identified within both three and four profile models. In both solutions, regression analyses and mean-level differences demonstrated that each profile was associated with adaptive versus maladaptive, and high-inhibitory outcomes, respectively. Specifically, in both three and four profile solutions, the resilient profile was associated with high Personal Wellbeing and interpersonal adjustment (measured by Perceived Quality of Interethnic Relations and Social Dominance Orientation), with the reverse trends apparent among brittle participants. Moreover, the overcontrol profile was associated with low Openness to Change values. The four profile solution included an additional profile with low-inhibitory personality tendencies characteristic of undercontrol, and was associated with high Openness to Change values. Consequently, the four profile solution was more 
representative of variability along Block and Block's (1980) ego-resilience and ego-control domains.

However, outcomes associated with Self-Enhancement values were not as clear cut. We expected differences in Self-Enhancement to be primarily linked to regulatory control, but in the three profile solution, Self-Enhancement was highest among resilient participants and lowest among overcontrol participants. Consistently, in the four profile solution, SelfEnhancement was highest among resilient participants, but there was no significant differences in Self-Enhancement between the remaining profiles. Consequently, these outcomes demonstrate a less coherent set of findings compared to previous research using six traits (Isler et al., 2016).

The results from the current study would suggest that the additional trait within the SixFactor Model (Honesty-humility) provides critical information and enhances the precision of resultant profiles. The advantage of using Honesty-humility in the identification of overcontrol versus undercontrol profiles is intuitively consistent with the outcomes associated with this trait; individuals scoring low in Honesty-humility tend towards greater self-promotion and risk-taking behaviours, and low impulse control (De Vries, de Vries, \& Feij, 2009; Weller \& Thulin, 2012). These findings are consistent with the spontaneous, sensation-seeking tendencies of undercontrollers, and enhance the overall explanatory power of personality profiles (see Isler et al., 2016).

\section{Limitations, Future Research, and Conclusions}

The generalizability of the current results is, of course, limited by the nature of the sample and personality scale used. Specifically, the current analyses were performed on the same sample as examined by Isler et al. (2016). Additionally, the short trait-markers used here may also come at the expense of trait complexity and predictive breadth (Messick, 1995). More detailed measures incorporate variance from several facets of personality within each trait, whereas trait markers tend to favour one facet to maximise alpha reliability. Further analyses should be performed on more varied samples, using more detailed personality measures, to establish the replicability of the present results.

At face value, the three profile model identified within the present research provides support for the replicability of a three profile solution as the minimum set of types necessary for an interpretable clustering of traits (see Asendorpf et al., 2001). However, the 
configurations and interpretations of the frequently reported three profile solution are inconsistent across studies (see Herzberg \& Roth, 2006). In our view, a likely explanation for the replicability problem in previous research may well be a product of the inadequacy of the three profile solution in contrast to a four profile solution.

Indeed, the current analyses demonstrate strong support for a four profile model as providing greater coherence and predictive ability as compared to an alternative three profile model, regardless of whether solutions are estimated using the Five or the Six-Factor Model of personality. These profiles likewise provide a more meaningful understanding of personality functioning, as individuals (rather than isolated traits) experience and engage with the environment (Donnellan \& Robins, 2003). Moreover, the theoretical interpretability of the four profile solution, as reflecting variation in self-regulatory processes (Block \& Block, 1980), provides insight into the mechanisms underlying personality development and expression. Thus, the current person-centred perspective provides an important and theoretically founded complement to the traditional variablecentered approach. 

STUDY 3. Validation of the Four Profile Model of Personality Types: Empirical Convergence with Ego-Constructs

\begin{abstract}
We examined exemplars of personality types identified by a four profile solution of personality traits to assess the proposed relationships between type-membership and egoconstructs. Selected participants, previously identified as representing resilient, brittle, overcontrol, and undercontrol profiles, were found to converge onto clearly differentiated domains of ego-resiliency and ego-control. Supplementary analyses found no evidence for a frequently proposed curvilinear relationship between the two ego-constructs. Results indicate that configurations of traits are best understood as reflecting four core components of either high or low levels of ego-resiliency and ego-control.
\end{abstract}




\section{Introduction}

Revitalisation of a person-centred approach to personality has emphasised the importance of within-person organization of personality attributes as a complement to a variablecentred methodology (Asendorpf, 2006). Central to the person-centred approach are Block and Block's (1980) self-regulatory dimensions: ego-resiliency and ego-control. Broadly explained, ego-constructs are generalised frameworks for experiencing and reacting to the environment, and promote individual differences in flexible and adaptive responding to environmental demands (ego-resiliency), and containment/suppression or expression of internal motivations (ego-control). The argument is commonly made that these selfregulatory systems have considerable explanatory power when applied to the patterning of personality traits (Gramzow et al, 2004).

This person-centred literature typically focuses on configurations of personality traits, often referred to as prototypes, types, or profiles. The most frequently identified solution reflects three types, leading researchers to often promote a three profile model as the minimum set necessary for an interpretable clustering of traits (Asendorpf et al., 2001; Robins et al., 1996). This pattern is consistent with a proposed curvilinear relationship between ego-control and ego-resiliency, whereby either high or low ego-control is linked to low levels of ego-resiliency (Asendorpf et al., 2001; Caspi, 1998).

In contrast to this standard interpretation of personality types, recent investigations have provided support for a four profile solution as a superior alternative (see Isler et al., 2016). Specifically, Isler, and colleagues have argued that the four profile solution preserves the unique effects of both ego-resiliency and control, rather than conflating high and low control with low ego-resiliency. Consistently, Isler et al. identified four distinct groupings of participants that were characteristic of high and low resiliency, and high and low control, respectively, using a combination of continuous-level and categorical methodologies. However, the extent to which this four profile solution truly reflects variation along Block and Block's (1980) ego-resiliency and ego-control dimensions was not directly tested.

\section{Relationships between Ego-Constructs}

The research on the empirical links between the ego-resiliency and ego-control dimensions presents a confusing picture. Various studies have demonstrated both positive and negative linear trends (often with moderating effects of age, gender, and ethnicity). For instance, 
Block (1993) found that ego-control and ego-resiliency were independent of one another in childhood among boys and girls, whereas in adolescence ego-resiliency was associated with higher levels of ego-control among girls only. In contrast, Chuang, Lamb, and Hwang (2006) found no relationship between ego-constructs in girls across ages, but found a positive correlation among young boys, and a negative correlation among teenage boys. Finally, Letzring et al. (2005) found ego-resiliency to be associated with lower levels of ego-control among both genders in a college sample (but this relationship was stronger among males). Moreover, correlates within their sample suggest that undercontrolling tendencies are associated with more maladaptive features in Caucasian, as compared to Asian-Americans, Hispanic, and African American participants. Investigations into the proposed quadratic relationship between the two variables provide similarly mixed results ${ }^{3}$. Although direct evidence of a curvilinear trend is sometimes demonstrated (Asendorpf \& van Aken, 1999; Gramzow et al., 2004), longitudinal investigations have found this trend at some age groups, but not others from the same sample (Eisenberg, Fabes, Gunthrie, \& Reiser, 2000; Eisenberg et al. 2003).

The mixed findings suggest that it is premature to conclude that the two constructs are represented by an inverse-U shaped relationship (Asendorpf et al., 2001). Additionally, the majority of previous investigations assess the relationship between ego-control and egoresiliency among young children and adolescents. As longitudinal studies demonstrate different developmental trajectories of both ego-constructs (Block, 1993; Chuang et al., 2006), it is important to test the proposed relationships in an adult sample, among whom personality characteristics should be more stable and less prone to developmental fluctuations.

\section{Current Investigation}

The primary objective of the current investigation was to assess the proposed relationship between Isler et al.'s (2016) four profile solution and measures of Block and Block's (1980) ego-constructs. To accomplish this, we targeted participants whose trait configurations were representative of Isler and colleague's four personality profiles, and retested these

\footnotetext{
${ }^{3}$ The authors note that a major problem in the identification of consistent trends in the literature is complicated by the nature of the tests conducted. For instance, studies investigating the curvilinear relationship between ego-resiliency and ego-control frequently do not directly test the relationship between the constructs, but instead employ alternative paradigms or measures to assess the proposed relationship (Flores, Cicchetti, \& Rogosch, 2005; Robins et al., 1996).
} 
participants using measures of ego-resiliency and ego-control. Along with our primary research goals, we directly analysed the relationship between ego-resiliency and egocontrol.

As previously discussed, the four profile model is conceptualised as preserving the unique effects of high and low ego-resiliency and ego-control, respectively. Accordingly, we predicted that membership within types should converge on unique outcomes of either high or low levels of ego-resiliency and ego-control (see Figure 3). Specifically, we predicted that resilient and brittle profiles would be associated with high versus low levels of egoresiliency, whereas overcontrol and undercontrol profiles would be associated with high versus low levels of ego-control. Furthermore, we predicted that resilient and brittle profiles would not differ from one another in terms of ego-control, and overcontrol and undercontrol profiles would likewise not differ from one another with regards to egoresiliency.

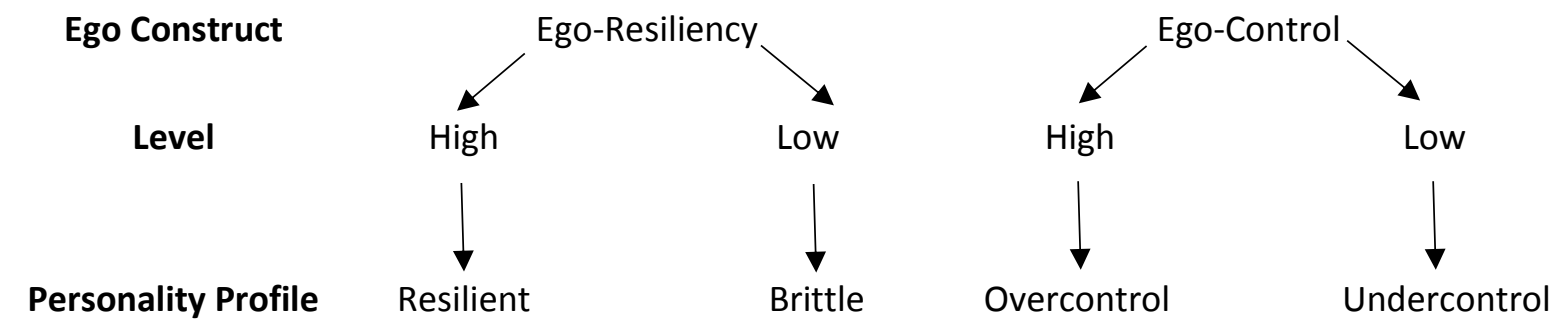

Figure 3: Proposed relationship between ego-constructs and personality profiles

Because of the prior contrasting findings, we had no direct predictions regarding the relationship between the dimensions of ego-resiliency and ego-control. Thus, we remained open to the possibility that ego-resiliency and ego-control would be unrelated, or related in a linear or curvilinear fashion. Notably, the current sample is unique in that it considers adult participants sampled from the general population.

\section{Method}

\section{Sample}

We analysed responses from 205 selected individuals who had previously participated in the New Zealand Attitudes and Values Survey. Participants were selected to take part in a follow-up survey as exemplars of their assigned profiles, with a goal of achieving 50 
participants per profile. Females represented the majority of participants (80.5\%). Mean age of the respondents was $50.81(S D=16.25)$.

Sampling procedures and composition. We combined the most recent personality scores from all participants who completed the first four waves of the New Zealand Attitudes and Values Survey to develop a large population-based sample of standard prototypical scores. The total number of participants within this combined sample was 10410. Latent Profile Analysis was performed on personality traits measured within this combined sample.

As expected, results mirrored the four profile pattern developed in the previous investigation (Isler et al., 2016). Two profiles differed in regards to exclusively high (desirable) and low (undesirable) scores, reflecting resilient and brittle participants, respectively. Additionally, two profiles varied in regards to high/low inhibitory traits: an overcontrol profile scored high in Honesty-humility, and low in Openness and Extraversion, whereas an undercontrol profile demonstrated the opposite trend. Entropy was moderate (.54), and average latent class probabilities demonstrated acceptable convergence within each profile; Resilient (.75), Brittle (.72), Overcontrol (.73), Undercontrol (.72). Based on estimated posterior probabilities, each profile also demonstrated adequately robust proportions of the total sample; Resilient (29.1\%), Brittle (10.2\%), Overcontrol (43.2\%), Undercontrol (17.5\%).

Using MPLUS software (Muthèn \& Muthèn, 2012) we calculate the class membership probability score for each participant. This reflected their respective convergence within each class. Prototypical members within each profile were identified, such that selected participants had a minimum of $50 \%$ or greater convergence with their assigned profile. Among these prototypical participants, those who had previously indicated willingness to be contacted for follow-up analyses were selected as the pool of potential participants for the current study. Mail-out surveys were distributed to participants, with a goal of achieving 50 participants from each of the four profiles; a projected total sample of 200.

Emphasis was placed on targeting participants with higher probability of profile membership. However, due to low response rates from some of the profiles, the lower limit of prototypicality was inconsistent across samples, resulting in a much higher average probability of class membership within the resilient profile. Despite lower scores among the 
brittle, overcontrol, and undercontrol profiles, the probability of class membership within these profiles was consistent with those within the larger sample, and are therefore interpretable. Details of the response rate and average probability of class membership for each of the profiles are included in Table 17.

Table 17

Prototypical respondents:

Response rate and average probability of class membership

\begin{tabular}{lcccc}
\hline & & & \multicolumn{2}{c}{ Probability of Class Membership } \\
\cline { 4 - 5 } & Responded & Response Rate & Mean & SD \\
\hline Resilient & 55 & .90 & .91 & .06 \\
Brittle & 42 & .71 & .76 & .16 \\
Overcontroller & 57 & .85 & .76 & .14 \\
Undercontroller & 51 & .75 & .73 & .15 \\
\hline$N=205$ & & & &
\end{tabular}

\section{Measures $^{4}$}

Ego-resiliency and ego-control. Self-report measures of ego-resiliency and ego-control developed by Jack Block were used here (for a review, see Letzring et al., 2005). The egoresiliency scale consisted of 14 positively-valenced items, and the ego-control scale consisted of 37 negatively and positively valenced items. Both were measured using 4 point Likert scales. Internal consistency reliability values in this study was acceptable for each factor: Ego-control $(a=.80)$, Ego-resiliency $(a=.80)$.

Mini-IPIP6. Participants completed the 5 factor 20-item Mini-IPIP (Donnellan et al., 2006), along with four items measuring a sixth trait, Honesty/humility. Items measuring this sixth trait were developed and validated by Sibley et al. (2011). Each personality item consists of a phrase evaluating a trait-relevant behaviour (e.g., 'I have a good word for everyone'), and is measured on a 7-point Likert scale.

An exploratory factor analysis (Appendix A) demonstrated that items loaded strongly onto the appropriate factors, with the exception of one Conscientiousness item 'I make a mess of things', which loaded moderately onto both Conscientiousness and Emotional Stability. Internal reliability for the Conscientiousness variable $(a=.60)$ was however acceptable, given the small number of items. Remaining reliability scores were all robust: Openness $(a=.78)$, Agreeableness $(a=.80)$, Extraversion $(a=.81)$, Emotional Stability $(a=$

\footnotetext{
${ }^{4}$ For scale items and instructions, view Appendix B
} 
$.79)$, and Honesty/humility $(a=.81)$. Reverse-items were recoded and scores for individual items were averaged to produce a total score for each factor.

\section{Procedure}

Before employing inferential statistics, we confirmed the appropriateness of the four profile solution within the present sample by conducting a Latent Profile Analysis of current participants' responses to the personality trait measures. We reviewed the fit and configuration of the resultant profiles, and assessed any transitions between profile memberships 5 . Notably, this analysis provided the added benefit of functioning as a manipulation check. As the selected participants are conceived as reflecting exemplars of each prototyple (with a degree of convergence within their assigned class of $50 \%$ or higher), we would expect that latent profiles presently identified would be more clearly differentiated than those obtained within the general population sample. Following this, we conducted a series of one-way ANOVAs to compare mean-level differences in ego-resiliency and ego-control between profiles, as well as follow-up t-tests to assess our predictions.

Finally, we considered the proposed curvilinear relationship between ego-control and egoresiliency.

\section{Results}

\section{Four Profile Solution: Trait Configurations and Model Fit}

Latent Profile Analyses were initially performed on the personality traits of the selected 205 participants using MPLUS software (Muthèn \& Muthèn, 2012). The Six-Factor Model of traits was used, rather than the Five-Factor Model, due to the superior fit and predictive ability of the resultant profile solutions (see Isler, Fletcher, Liu, \& Sibley, 2017; Isler et al., 2016). As expected, the four profile solution was consistent with the four profiles previously identified. The resilient profile was characterised by high (or desirable) mean values on each personality trait, whereas the brittle profile was characterised by exclusively low (or undesirable) scores on all traits. In terms of the control dimension, overcontrollers

\footnotetext{
${ }^{5}$ Less than two percent of participants were reclassified into different profiles as a result of the Latent Profile Analysis. Comparative tests were done to assess outcomes where we: 1) omitted the participants, 2) included them within their original category, and 3) included them in the updated profile category. As outcomes did not change depending on the placement of these individuals, we included all participants within their final profile category, as identified by the current Latent Profile Analyses.
} 
demonstrated low Extraversion, low Openness, and high Honesty-humility, with the reverse pattern apparent among undercontrollers.

The entropy of the current solution was robust (.81), and average latent class probabilities demonstrated strong convergence within each profile; Resilient (.92), Brittle (.95), Overcontrol (.84), Undercontrol (.93). These fit indices were consistent with an understanding of the current participants as representing exemplars converging on welldifferentiated personality types derived from a population-based sample. Profile trait configurations are presented in Figure 4.

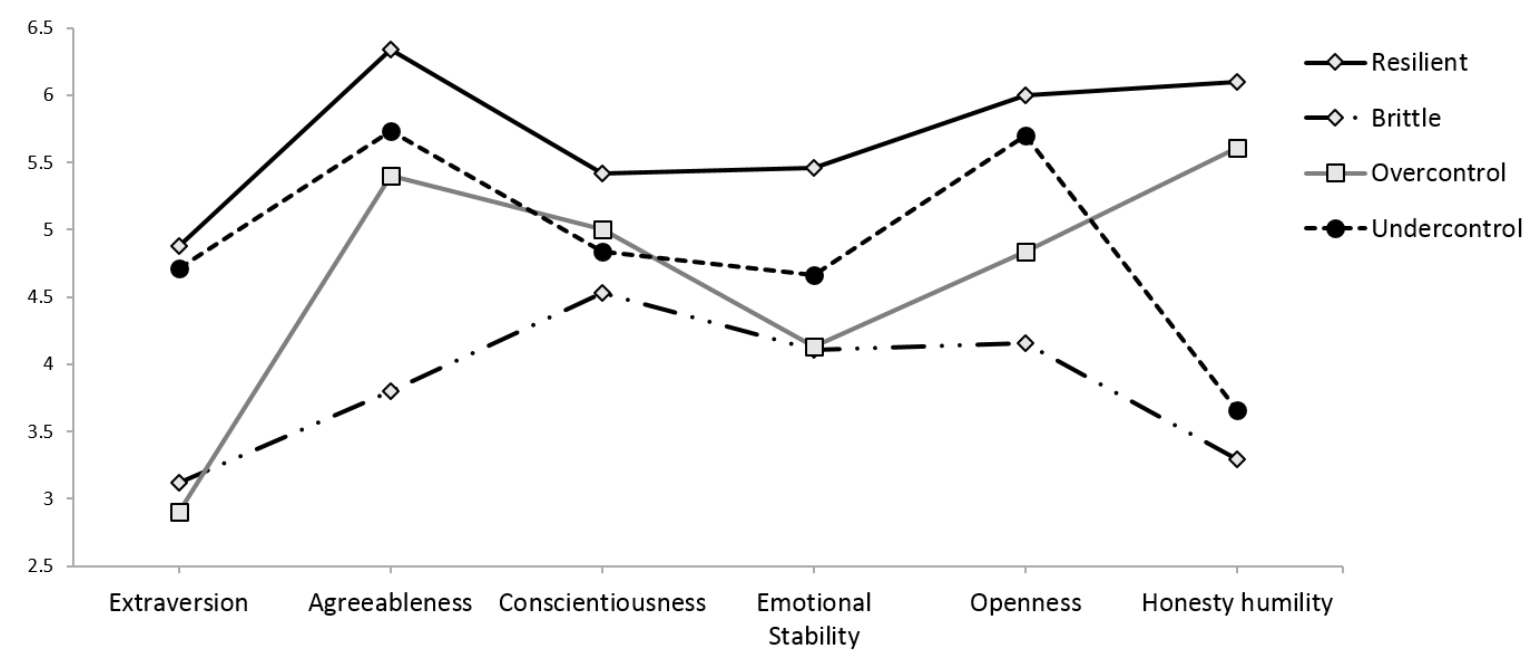

Figure 4. Four profile solution among prototypical respondents

\section{Personality Profiles and Ego-Constructs}

Group-level means and standard deviations for both ego-dimensions were calculated for each of the four profile categories (Table 18). Mean trends provided initial support for our predictions, whereby resilient and brittle profiles were associated with the highest and lowest levels of ego-resiliency, and overcontrol and undercontrol profiles exhibited the highest and lowest levels of ego-control.

Table 18

Means and standard deviations of ego-constructs as a function of personality profiles

\begin{tabular}{lllllllll}
\hline & \multicolumn{3}{c}{ Ego-Resiliency } & & \multicolumn{3}{c}{ Ego-Control } \\
\cline { 2 - 4 } \cline { 7 - 8 } Personality Profile & $M$ & $S D$ & $N$ & & $M$ & $S D$ & $N$ \\
\hline Resilient & 3.29 & .35 & 54 & & 2.57 & .31 & 54 \\
Brittle & 2.81 & .40 & 42 & & 2.60 & .28 & 42 \\
Overcontrol & 2.90 & .34 & 55 & & 2.71 & .31 & 55 \\
Undercontrol & 3.00 & .30 & 54 & & 2.47 & .28 & 54 \\
\hline Note: $M=$ Mean; $S D=$ standard deviation; $N$ = number of participants
\end{tabular}


A series of one-way between-subjects ANOVAs were conducted comparing resilient and brittle, as well as overcontrol and undercontrol, profiles to examine differences in egoresiliency and ego-control scores. Levene's test of homogeneity of variance demonstrated no significant difference in variances between the profiles in regards to either ego-resiliency $(p=.390)$, or ego-control $(p=.917)$. Results of the ANOVAs indicated that there were significant mean-level differences between the four profiles in relation to both egoresiliency $\left(F(3,201)=19.08, p<.001, \eta^{2}=.28\right)$ and ego-control $\left(F(3,201)=5.79, p=.001, \eta^{2}=\right.$ $.09)$.

Tests of planned comparisons were run to assess our hypotheses regarding the underlying nature of these mean level differences. As predicted, resilient participants scored significantly higher in ego-resiliency as compared to brittle participants $t(201)=6.81, p<$ .001 , with no difference in ego-control $t(201)=.62, p=.539$. Additionally, overcontrollers scored significantly higher in ego-control as compared to undercontrollers $t(201)=4.11, p<$ .001 , with no difference in ego-resiliency $t(201)=1.68, p=.094$. This supports the hypothesis that the personality patterns originally identified by Isler et al. (2016) converge on four poles of high/low ego-resiliency and high/low ego-control dimensions.

Finally, we tested the possible linear and curvilinear relationship between ego-control and ego-resiliency. We found a small, marginally significant negative correlation between the two constructs $(r=-.13, p=.055)$. The quadratic component did not add any additional variance to the linear component: $R^{2}$ change $=.00, F_{\text {change }}(1,202)=0.00, p=.988$. This is inconsistent with the standard model in which high and low control are associated with maladaptive functioning (see Asendorf \& van Aken, 1999; Robins et al., 1996).

\section{Discussion}

Prior investigations provide support for a four profile solution of personality, which is a more plausible and systematic match to Block and Block's (1980) two dimensional egoconstructs as compared to the commonly identified three profile solution (Isler et al., 2016; Isler et al., 2017). However, the extent to which this four profile solution reflects variation along ego-resiliency and ego-control dimensions had not been previously directly tested. The current investigation addressed this issue by resampling exemplars of resilient, brittle, overcontrol, and undercontrol participants identified by Isler et al. (2016), retesting their personality scores, and analysing their links with Block and Block's (1980) ego-constructs. 
Supplementary analyses were also conducted to consider the relationships between the ego-control and ego-resiliency constructs.

\section{Major Findings}

Findings confirmed that the four profile solution of high/low resilient/control personality types identified by Isler et al. (2016) reflected high and low levels of Block and Block's (1980) ego-resiliency and ego-control constructs. Specifically, overcontrollers scored higher in egocontrol as compared to undercontrollers, whereas resilient participants scored higher in ego-resiliency compared to brittle participants. Moreover, adding valuable discriminant validity, over/under controllers did not differ from one another with regards to egoresiliency, and resilient/brittle participants did not differ from one another in terms of egocontrol. Finally, we found no evidence of either a significant linear or a curvilinear trend between ego-resiliency and ego-control. Taken together, the current results provide strong evidence for Block and Block's (1980) self-regulatory processes as being reflected within four core configurations of personality.

\section{Implications, Limitations, and Future Directions}

The interpretation of the four profile model is based on Kremen and Block's (1998) criticisms of the tendency to conflate either high or low control with maladaptive functioning. Indeed, both the theoretical literature and subsequent investigations frequently highlight both the maladaptive and the adaptive features of high and low egocontrol (Block \& Block, 1980; Gramzow et al., 2004; Letzring et al., 2005). Accordingly, researchers have emphasised the unique benefits of both ego-constructs in predicting individual differences in either a) containment and expression of impulse (in the case of egocontrol), or b) psychological adjustment and wellbeing (in the case of ego-resiliency) (Letzring et al., 2005).

The present investigation supports the conclusion that differences in personality profiles and ego-constructs are best understood as reflecting core aspects of high and low ego-control and ego-resiliency. Although this is not incompatible with the assertion that extreme levels of ego-control may result in an inability to modulate behaviour (and thereby low-resiliency), it does suggest that over- and undercontrol tendencies should not be understood as inherently maladaptive. Our findings suggest that the three profile model overlooks important theoretical distinctions between profiles as embodied in a four profile 
model. We suggest that future studies should examine a four profile solution as a plausible and potentially superior alternative to a three profile solution.

However, the way in which our sample was collected (reflecting prototypical participants within each profile), suggests that caution should be exercised in generalizing our findings to the general population. Indeed, Block and Block (1980) suggested that extreme characteristics of either control tendency should manifest as non-adaptive, unmodulated behaviour. As the current participants represent exemplars of each profile based on group-level averages, it is likely that the most extreme cases of control and resiliency are not presently reflected. Likewise, the absence of a curvilinear relationship between ego-domains in the present investigation may be an outcome of the characteristics of the current sample.

Nevertheless, the clear relationship between ego-constructs and personality systems identified here indirectly supports the viability of a developmental framework for personality, and provides an avenue to more directly consider the psychosocial antecedents of the expression of personality traits. Ego-structures develop as the individual faces and responds to different contextual demands and opportunities, and can be conceptualised as being expressed within an individual personality system (Block \& Block, 1980; Block \& Kremen, 1996; Kremen \& Block, 1998). Consistently, we suggest that the configurations of traits identified in this study may be usefully interpreted as self-regulatory systems designed to integrate internal motivations with external demands. 



\section{General Discussion}

The present investigations addressed the formalization and empirical assessment of theoretically founded typological structures of personality. Consistent with previous studies, Block and Block's (1980) theory of ego-resiliency and ego-control was the foundation for developing and interpreting configurations of personality traits. Although the three profile model is generally accepted as reflecting the minimum number of types necessary for a generalizable solution (Robins et al., 1996), the marked heterogeneity of profile structures between studies suggests that the widespread adoption of a three profile solution is premature (Herzberg \& Roth, 2006). Moreover, a three profile model overlooks key distinctions that are more readily apparent within a four profile configuration of traits (Barbaranelli, 2002; Gramzow et al., 2004). Accordingly, comparisons between three and four profile models are at the core of the current investigation.

The arguments for both three and four profile solutions are founded in two competing interpretations of Block and Block's (1980) theory of ego-constructs in terms of two key dimensions: ego-resiliency and ego-control. Broadly explained, ego-resiliency reflects an individual's adaptive capacity to recognise and adjust to changing circumstances, whereas ego-control is indicative of an individual's expression or containment of internal motivations and impulses. The three profile model, as noted previously, conflates both high and low control with maladaptive functioning (low ego-resiliency), thereby embodying a proposed curvilinear relationship between the two constructs (Asendorpf et al., 2001; Block \& Block, 1980). In contrast, the four profile model emphasises the roles of ego-resiliency and egocontrol as distinct mechanisms with independently meaningful outcomes (Kremen \& Block, 1998; Letzring et al., 2005). Notably, from this perspective, variation along ego-control is associated with both maladaptive and adaptive features (Block \& Block, 1980).

I conducted a series of studies to critically examine the three and four profile solutions within a large, population-based dataset. Starting within the Six-Factor Model of personality, I compared the typically identified three profile solution against an alternative four profile solution using Latent Profile and Latent Transition techniques. I applied statistical and theoretical considerations to assess both models as meaningful frameworks for profile development in the domains of 1) model fit; 2) longitudinal stability; 3) configural interpretability; and 4) predictive ability. Following this, I examined the replicability of both solutions within the traditional Five-Factor Model of personality, and considered the utility 
of using the Six- as opposed to Five-Factor Model of personality for profile development. Finally, I assessed the proposed relationship between the most substantiated type-solution and ego-domains using direct measures of ego-control and ego-resilience.

\section{Summary of Findings}

Using the Six-Factor Model as a basis for profile development, I found evidence for good model fit and longitudinal stability for both three and four profile solutions. The configurations of traits in both models (Figures 1a and $1 \mathrm{~b}$ ) were likewise interpretable according to their respective theoretical premises. Specifically, within the four profile model, one profile was characterised by exclusively high (desirable) trait scores (suggesting high resilience), whereas another was characterised by the opposite pattern (suggesting low resilience). Additionally, a low-inhibitory (undercontrol) profile was characterised by high Extraversion and Openness, with low Honesty-humility, whereas a high-inhibitory (overcontrol) profile demonstrated the opposite trend. Comparatively, the configurations of traits within the three profile model mirrored results from the high-resilient and overcontrol profiles previously described, along with a third low-inhibitory (undercontrol) profile with generally moderate scores on all traits, and relatively higher levels of Openness to Experience and Extraversion, but with lower values on Honesty-humility.

Analyses of the predictive ability of type membership provided support for the four profile solution as a meaningful and necessary distinction beyond the three profile solution. Specifically, within this model, resilient and brittle profiles were associated with high/low adjustment outcomes, whereas overcontrol and undercontrol profiles were associated with high/low inhibition outcomes. These relationships suggest theoretically distinct egomechanisms underlying variation along type domains (Letzring et al., 2005). Comparatively, the predictive ability of the three profile solution was less interpretable; resilient and overcontrol profiles within this model were related to adjustment and inhibition, respectively, whereas the undercontrol profile was related to both low adjustment and low inhibition. For the profile configurations to illustrate a curvilinear relationship, we would expect both high and low ego-control to correspond with low adjustment. However, rather than a curvilinear relationship, the current pattern of relationships suggest that a three profile solution conflates low resilience with low control tendencies. 
Follow up analyses using the Five-Factor Model of personality demonstrated similar outcomes. Although both three and four profile models were associated with acceptable model fit, and interpretable configurations of traits (Figures $2 a$ and $2 b$ ), the four profile solution provided clearer predictive ability and theoretical interpretability compared to the alternative three profile solution. These results suggest that, regardless of whether the Five- or Six-Factor Model of personality provides the basis for type development, the four profile model provides a more coherent solution of personality types than the alternative three profile solution. However, the omission of the sixth trait (Honesty-humility) resulted in a reduction in profile precision and predictive ability. Particularly, inhibitory outcomes relating to undercontrol and overcontrol profiles were less clear-cut as compared to those developed using the more detailed Six-Factor Model. This finding is consistent with the role of Honesty-humility in accounting for variance relating to individual differences in impulsiveness and inhibition (De Vries et al., 2009; Weller \& Tikir, 2011; Weller \& Thulin, 2012), and enhances the explanatory power of the Six-Factor Model.

Finally, using the more precise configurations identified by the Six-Factor Model of personality, I confirmed that prototypical exemplars of the four profile types converge on clearly differentiated domains of ego-resiliency and ego-control. Specifically, resilient participants scored higher in ego-resiliency as compared to brittle participants, with no difference in ego-control. Moreover, overcontrollers scored higher in ego-control than undercontrollers, with no difference in ego-resiliency. This represents a convincing set of convergent and discriminant results. An assessment of the possible curvilinear relationship between the two ego-constructs likewise demonstrated no linear or curvilinear trend, providing good evidence that the two domains reflect independent mechanisms (Letzring et al., 2005).

\section{Complementary Functioning of Types and Traits}

Trait and type theories are organised around two different levels of explanation. Traittheories reflect classificatory systems that effectively describe inter-individual differences, but fail to account for, or explain, personality as a dynamic generative process. Conversely, typological perspectives reflect strategies for explaining personality functioning via underlying psychological systems. Although within- and between-person theorizing are typically interpreted as operating in opposition, modern type investigations emphasise the 
complementary functioning of trait and type approaches by targeting the intraindividual structure of traits working as coordinated systems. This approach considers the role of underlying causal mechanisms in producing both within-person variation and betweenperson differences across the observed trait dimensions (John \& Srivasta, 1999). Thus, a major benefit of the modern type approach is that is goes beyond purely descriptive systems of personality to a deeper level of explaining personality systems.

For example, Block and Block (1980) conceptualised ego-constructs as self-regulatory structures that develop over time as the individual experiences and engages with the social environment. Through repeated encounters, the individual develops a working model for perceiving and reacting to external conditions, which is then internalised into a stable system of affect and cognition. Notably, these self-regulatory systems recruit lower level subsystems which form the basic components for interpreting and relating to the world (Kremen \& Block, 1998). Consequently, self-regulatory ego-constructs should be reflected within interpretable intra-individual patternings of personality traits (Gramzow et al., 2004), which account for the primary dimensions along which variation in personality will occur (John, 1990).

Taken together, the current findings lend support for the presence of stable configurations of personality working within the individual as coordinated self-regulatory systems, which are causal generative mechanisms. The observed trait configurations within the four profile model (Study 1 ) were readily interpretable according to the theoretical literature on ego-resiliency and ego-control, and each profile predicted theoretically consistent outcomes in the domains of adaptive/maladaptive and inhibited/uninhibited functioning. Finally, prototypical exemplars of the four profile types (Study 3) reflected clearly differentiated domains of ego-resiliency and ego-control. Thus, the present results support the existence of a hierarchical patterning of personality in which internal selfregulatory mechanisms help generate the expression and functioning of personality typologies.

\section{Personality Types within Context}

An advantage of typological investigations of personality is in the focus of the whole person interactively engaged in a social world. Stated another way, these intra-individual systems not only develop through interactive encounters with the social environment, but continue 
to coordinate particular ways of experiencing and responding within complex interpersonal settings. Personality systems of interrelated traits should therefore result in systematically distinct ways of perceiving and engaging with the external world (Robins \& Tracy, 2003).

As an example, the overcontrol profile is characterised by low Extraversion and Openness, and high Honesty-humility. This configuration should produce the tendency towards unassertiveness, discomfort with ambiguity, and low motivation for self-promotion. Thus, when the outcome of a behaviour is uncertain but important (e.g. requesting a raise) this person should experience anxiety and procrastination. In contrast, an undercontroller, whose personality system contains high Extraversion and Openness, and low Honestyhumility, will likely experience low anxiety and optimistically push for a higher salary.

The adoption of the type approach to personality reflects the proposition that the way individuals respond to environmental situations depends on their unique configuration of traits, not just according to where they stand on a single trait. For instance, resilient individuals possess high Honesty-humility, much like overcontrollers, and should therefore tend towards low levels of self-promotion. Conceivably, this individual trait could produce inhibitory behaviour in the hypothetical job-promotion scenario discussed above. However, relatively high levels of resilience are also associated with high levels of Extraversion and Openness. These latter traits are associated with greater levels of assertiveness and comfort with ambiguity, which could promote a tendency towards less inhibitory constraint in the context of requesting a promotion.

Thus, knowledge of coordinated personality structures provides insight into frameworks of experience and action, and promotes the applicability of personality research by studying individuals as whole entities within contexts. Moreover, to the extent that personality types are based within a clear theoretical foundation, researchers should be able to develop a strong rationale for generating hypotheses about the type-based differences of experience and action (Robins \& Tracy, 2003). This has particular relevance in the domain of health psychology. Specifically, an understanding of the within-person dynamic process of experiencing and relating to the external environment can; 1) facilitate the identification of unique protective and risk factors associated with different typologies, 2) isolate the processes and mechanisms that mediate the relationship between type-membership and (mal)adaptive outcomes, 3) provide suggestions for targeted and specialised treatment strategies. 
As an example, overcontrollers and undercontrollers are associated with greater internalizing and externalizing problems, respectively (Huey \& Weisz). Although the general tendency to express or inhibit internal motivations and impulses provides an interpretable theoretical explanation for these relationships, the identification of more proximal mediators would provide a more direct and applicable set of findings. For instance, if the link between undercontrol and drug use (Block, Block \& Keyes, 1988) is mediated by a greater tendency towards spontaneous sensation seeking, then treatment strategies among these individuals may benefit from a focus on the development of more purposive and reflective thinking strategies.

\section{Typeness}

Another advantage of the current analyses is the assessment of profile-membership using a mixture of both continuous and categorical methodologies. Specifically, personality types were derived through Latent Profile Analysis, which assigns individuals into groups based on observed patterns of traits, while accounting for uncertainty of class membership (Nylund et al., 2007). Using this approach, profile-membership simultaneously maximises differences between types, and minimises the mean individual difference from the best fitting type. Resultant configurations of traits reflect the mean scores of responses within each grouping, and the likelihood of an individual being assigned to any given group is measured in terms of degree.

Interpreting types in terms of fuzzy sets, with permeable borders, provides a meaningful interpretation of personality typologies, which is consistent with personality development. Specifically, the personality types assessed in these studies reflect selfregulatory strategies which are outcomes of many reciprocal interactions between individuals and their environments. Thus, the diversity of experiences inherent in personenvironment transactions produces variability in both the types of self-regulatory strategies adopted and the extent to which individuals endorse these core strategies. This perspective was supported in the current analyses. For example, the types identified in Study 1 converged onto clearly identified personality profiles; $25 \%$ of participants were highly typical of their assigned profiles at a probability of class membership of $90 \%$ or greater. However, the degree of convergence within assigned profiles varied across individuals, with an average posterior probability of class membership within each profile ranging from 70 to 
75 percent (Table 2). Consequently, although underlying generative mechanisms explain centralised tendencies of personality configurations, the level of prototypicality within each type exists on a spectrum.

Beyond the theoretically appealing move away from completely discrete types, the present techniques provide some statistical and methodological advantages. Notably, by considering participants' probability of class membership, we can consider the extent to which the degree of convergence across multiple types reflects interpretable outcomes. Accordingly, we can gain incremental information by using sensitive continuous measures that take into account the extent to which individuals' personality structures are aligned to core prototypical types.

Extensions of the typeness approach. The current investigation has emphasised the unique roles of each personality type by considering prototypical exemplars, and degrees of convergence with individual types. However, as discussed above, an individual may demonstrate convergence across multiple types. For instance, closer inspection of an individual classified as an undercontroller may demonstrate a more complex pattern whereby profile membership converges $60 \%$ with the undercontrol prototype, and $40 \%$ with the resilient prototype.

Future investigations would benefit by considering this within-person configurations of typeness, which could provide avenues for investigating meaningful overlap between type membership (Block \& Block, 1980). This has particular theoretical value, as an individual's tendency towards either high or low ego-resiliency is reflected in either the adaptive or maladaptive expression of ego-control (Block \& Block, 1980). In this manner, the adaptive features of both low and high ego-control will be expressed amongst individuals with relative high ego-resiliency, whereas maladaptive features of both control strategies will be emphasised amongst individuals with low ego-resiliency. Because probabilities of class membership across profiles are completely dependent (the sum of each participant's probabilities across profiles must sum to 1.00), profiles cannot be combined to predict outcomes. However, by specifically targeting and selecting groups of participants with similar within-person degree-of-convergence across multiple types, researchers may be able to gain a more nuanced understanding of the within-person interactive framework of resiliency and control addressed in the literature. 


\section{Limitations and Future Research}

Little research has examined factors influencing statistical power to detect the correct number of latent classes using Latent Profile Analysis. However, there is some evidence that the distance between latent classes plays a major role in model identification. Notably, when the inter-class distance is small, fit indices tend to favour fewer classes, especially when coupled with few indicators or small sample sizes (Tein, Coxe, \& Cham, 2013). In the present case, the notable (though interpretable) overlap between latent profiles could therefore obscure the correct identification of number of classes, especially with small samples.

The need for large sample sizes increases the costs for conducting studies and may restrict the ability of researchers to apply or extend the current findings. A potential solution to this problem lies in the further examination and replication of the four profile solution, albeit with initially large samples. Through repeated investigations, researchers can identify essential trends in personality profiles that most effectively differentiate between profile structures, and develop probability-based algorithms to delineate the within-person composition of personality typeness. These algorithms could then be applied to relatively small sample sizes for further research.

Finally, the present analyses were conducted on a large, population based sample, and are therefore highly generalizable. However, only one country (New Zealand) was investigated. As self-regulatory structures develop in reciprocal coordination with the external and interpersonal environment, it is likely that differences in socio-cultural contexts will impact the individual's self-regulatory development and functioning. For instance, relatively high levels of undercontrol may be more prevalent and lead to more advantageous outcomes within a culture that promote high levels of independence and selfexpression. However, the same level of undercontrol may not be as prevalent or advantageous within a culture that emphasises reserved functioning and self-regulation. Cross-cultural investigations are therefore needed to investigate the replicability of the four profile solution, as well as the potential for invariance of profile structures and typemembership outcomes. 


\section{Conclusion}

The present series of investigations demonstrate strong support for a four profile solution of personality types as embodying variation along Block and Block's (1980) ego-domains. These findings reflect a theoretically interpretable model, whereby higher-order ego-constructs generate and organise lower-level systems of personality functioning. Specifically, egoconstructs reflect stable self-regulatory strategies which integrate internal motivations with external demands. These systems develop as the individual faces and responds to different contextual demands and opportunities, and can be conceptualised as being expressed within an individual personality system (Block \& Block, 1980; Block \& Kremen, 1996; Kremen \& Block, 1998).

These studies provide a coherent set of findings that serve as an effective complement to the variable-centred approach to personality. Although the modern trait approach effectively organises the between-person variation manifested within personality, it can be criticised for not accounting for the whole person, or providing an explanatory model of personality functioning (Block, 1995; Block, 2010). By targeting the role of underlying causal mechanisms in producing both within-person variation and between-person differences across observed trait dimensions, the present studies advance a theoretical foundation with which to organise, explain, and interpret otherwise descriptive systems of personality. Thus, the proposed relationship between ego-constructs and intra-individual structures of personality traits provides an avenue to more directly consider the psychosocial antecedent and functioning of personality traits as they relate to the whole person. 



\section{References}

Asbrock, F., Sibley, C. G., \& Duckitt, J. (2010). Right-wing authoritarianism and social dominance orientation and the dimensions of generalized prejudice: A longitudinal test. European Journal of Personality, 24, 324-340.

Asendorpf, J.B. (2006). Typeness of personality profiles: A continuous person-centred approach to personality data. European Journal of Personality, 20, 83-106.

Asendorpf, J. B., Borkenau, P., Ostendorf, F., \& van Aken, M. A. G. (2001). Carving personality description at its joints: Confirmation of three replicable personality prototypes for both children and adults. European Journal of Personality, 15, 169198.

Asendorpf, J. B., \& van Aken, M. A. G. (1999). Resilient, overcontrolled, and undercontrolled personality prototypes in childhood: Replicability, predictive power, and the traittype issue. Journal of Personality and Social Psychology, 77, 815-832.

Asendorpf, J.B., van de Schoot, R., Denissen, J.J.A., \& Hutteman, R. (2014). Reducing bias due to systematic attrition in longitudinal studies: The benefits of multiple imputation. International Journal of Behavioural Development, 38(5), 453-460.

Ashton, M. C., \& Lee, K. (2005). Honesty-humility, the Big Five and the Five-Factor model. Journal of Personality, 73, 1321-1353.

Ashton, M.C., \& Lee, K. (2008). The prediction of Honesty-Humility-related criteria by the HEXACO and Five-Factor models of personality. Journal in Personality, 42, 12161228.

Ashton, M.C., Lee, K., Perugini, M., Szarota, P., De Vries, R.E., Di Blas, L., ... De Raad, B. (2004). A six-factor structure of personality-descriptive adjectives: Solutions from psycholexical studies in seven languages. Journal of Personality and Social Psychology, 86, 356-366.

Ashton, M.C., Paunonon, S.V., \& Lee, K. (2014). On the validity of narrow and broad personality traits: A response to Salgado, Moscoso, and Berges (2013). Personality and Individual Differences, 56, 24-28. 
Barbaranelli, C. (2002). Evaluating cluster analysis solutions: An application to the Italian NEO Personality Inventory. European Journal of Personality, 16, S43-S55.

Barron, F. (1953). An Ego-strength scale which predicts response to psychotherapy. Journal of Consulting Psychology, 5, 327-333.

Bauer, D., \& Curran, P. (2004). The integration of continuous and discrete latent variable models: potential problems and promising opportunities. Psychological Methods, 9, 3-29.

Berry, J., Elliott, T., \& Rivera, P. (2007). Resilient, undercontrolled, and overcontrolled personality prototypes among persons with spinal cord injury. Journal of Personality Assessment, 89, 292-302.

Block, J. (1971). Lives through time. Berkeley: Bancroft Books.

Block, J. (1993). Studying personality the long way. In D.C. Funder, R.D. Parke, C. TominsonKeasey, \& K. Wideman (Eds.), Studying lives through time: Personality and development (pp. 9-41). Washington, DC: American Psychological Association.

Block, J. H. (1995). A contrarian view of the five-factor approach to personality description. Psychological Bulletin, 117, 187-215.

Block, J. (2002). Personality as an affect-processing system: Toward an integrative theory. Mahwah, NJ: Erlbaum.

Block, J. H. (2010). The Five-Factor Framing of personality and beyond. Some Ruminations. Psychological Inquiry, 21, 2-25.

Block, J. H., \& Block, J. (1980). The role of ego-control and ego-resiliency in the organization of behavior. In W. A. Collins (Ed.), Minnesota symposium on child psychology (Vol. 13, pp. 39-101). Hillsdale, NJ: Erlbaum.

Block, J., \& Kremen, A. M. (1996). IQ and ego-resiliency: Conceptual and empirical connections and separateness. Journal of Personality and Social Psychology, 70, 349361.

Block, J. H., \& Martin, B. (1955). Predicting the behavior of children under frustration. Journal of Abnormal and Social Psychology, 51, 281-285. 
Bobo, L. (1998). Race, interests, and beliefs about affirmative action. American Behavioural Scientist, 41, 985-1003.

Boies, K., Lee, K., Ashton, M. C., Pascal, S., \& Nicol, A. A. M. (2001). The structure of the French Personality Lexicon. European Journal of Personality, 15, 277-295.

Caprara, G.V., \& Cervone, D. (2000). Personality: Determinants, Dynamics, and Potentials. New York: Cambridge University Press.

Caspi, A. (1998). Personality development across the life course. In W. Damon (Series Ed.) \& N. Eisenberg (Vol. Ed.), Handbook of child psychology: Vol. 3. Social, emotional, and personality development (311-388). New York: Wiley.

Caspi, A., \& Silva, P. (1995). Temperamental qualities at age 3 predict personality traits in young adulthood: Longitudinal evidence from a birth cohort. Child Development, 66, 486-498.

Cattell, R.B. (1943). The description of personality: Basic traits resolved into clusters. Journal of Abnormal and Social Psychology, 38, 476-506.

Causadias, J., Slavatore, J., Sroufe, A. (2012). Early patterns of self-regulation as risk and promotive factors in development: a longitudinal study from childhood to adulthood in high-risk sample. International Journal of Behavioral Development, 36, 293-302.

Chuang, S.S., Lamb, M.E., \& Hwang, C.P. (2006). Personality development from childhood to adolescence: A longitudinal study of ego-control and ego-resiliency in Sweden. International Journal of Behavioral Development, 30, 338-343.

Clark, S., \& Muthén, B. (2009). Relating latent class analysis results to variables not included in the analysis. Submitted for publication.

Cleland, C.M., Rothschild, L., \& Haslam, N. (2000). Detecting latent taxa: Monte Carlo comparison of taxometric, mixture model, and clustering procedures. Psychological Reports, 87, 37-47.

Costa, P. T., Jr., Herbst, J. H., McCrae, R. R., Samuels, J., \& Ozer, D. J. (2002). The replicability and utility of three personality types. European Journal of Personality, 16, Special Issue S1, 73-87. 
Costa, P.T., Jr., \& McCrae, R.R. (1988). From catalog to classification: Murray's needs and the five factor model. Journal of Personality and Social Psychology, 55, 258-265.

Costa, P. T. Jr, \& McCrae, R. R. (1992). Revised NEO personality inventory manual. Odessa, FL: Psychological Assessment Resources.

Costa, P.T., Jr., \& McCrae, R.R. (1995). Domains and facets: Hierarchical personality assessment using the revised NEO Personality Inventory. Journal of Personality Assessment, 64, 21-50.

Cummins, R.A., Eckersley, R., Pallant, J., van Vugt, J. \& Misajon, R. (2003). Development of a national index of subjective wellbeing: The Australian Unity Wellbeing Index. Social Indicators Research, 64,159-190.

De Fruyt, F., Mervielde, I., \& Van Leeuwen, K. (2002). The consistency of personality type classification across samples and fivefactor measures. European Journal of Personality, 16, S57-S72.

De Raad, B. (1992). The replicability of the Big Five personality dimensions in three wordclasses if the Dutch language. European Journal of Personality, 6, 15-29.

De Raad, B., Barelds, D. P. H., Levert, E., Ostendorf, F., Mlacić, B., Di Blas, L., . . Katigbak, M. S. (2010). Only three factors of personality description are fully replicable across languages: A comparison of 14 trait taxonomies. Journal of Personality and Social Psychology, 98, 160-173.

De Raad, B., \& Szirmak, Z. (1994). The search for the "Big Five" in a non-Indo-European language. The Hungarian trait structure and its relationship to the EPQ and the PTS. European Review of Applied Psychology, 44, 17-24.

De Vries, R. E., De Vries, A., \& Feij, J. A. (2009). Sensation seeking, risk-taking, and the HEXACO model of personality. Personality and Individual Differences, 47, 536-540.

De Vries, R.E., De Vries, A., De Hoogh, A., \& Feij, J. (2009). More than the Big Five: Egoism and the HEXACO model of personality. European Journal of Personality, 24, 635-654.

Di Blas, L., \& Forzi, M. (1998). An alternative taxonomic study of personality -descriptive attributes in the Italian language. European Journal of Personality, 12, 26-34. 
Di Blas, L., \& Forzi, M. (1999). Refining a descriptive structure of personality attributes in the Italian language: The abridged Big Three circumplex structure. Journal of Personality and Social Psychology, 59, 1216-1229.

Diener, E., Emmons, R. A., Larsen, R. J., \& Griffin, S. (1985). The Satisfaction with Life Scale. Psychological Assessment, 49, 71-75.

Digman, J.M. (1990). Personality structure: Emergence of the Five-Factor Model. Annual Review of Psychology, 41, 417-440.

Donnellan, B.M., \& Robins, R.W. (2010). Resilient, overcontrolled, and undercontrolled personality types: Issues and controversies. Social and Personality Psychology Compass, 4, 1070-1083.

Donnellan, M.B., Frederick, L., Oswald, B.M.B, \& Lucas, R.E. (2006). The Mini-IPIP scales: Tiny-yet effective measures of the Big Five factors of personality. Psychological Assessment, 18, 192-203.

Dubas, J.S., Gerris, J.R., Janssens, J.M., \& Vermulst, A.A. (2002). Personality types of adolescents: Concurrent correlates, antecedents, and type $\mathrm{X}$ parenting interactions. Journal of Adolescence, 25, 79-92.

Duckitt, J., \& Sibley, C. G. (2007). Right-wing authoritarianism, social dominance orientation and the dimensions of generalized prejudice. European Journal of Personality, 21, 113-130.

Eisenberg, N., Fabes, R.A., Guthrie, I.K., \& Reiser, M, (2000). Dispositional emotionality and regulation: Their role in predicting quality of social functioning. Journal of Personality and Social Psychology, 78, 136-157.

Eisenberg, N., Valiente, C., Fabes, R.A., Smith, C.L., Reiser, M., Shepard, S.A., et al. (2003). The relations of effortful control and ego-control to children's resiliency and social functioning. Developmental Psychology, 39, 761-776.

Ekehammar, B., \& Akrami, N. (2003). The relation between personality and prejudice: A variable- and a person-centred approach. European Journal of Personality, 17, 449464. 
Eysenck, H. J., \& Eysenck, S. B. G. (1969). Personality structure and measurement. San Diego: Knapp.

Fiske. D. W. (1949). Consistency of the factorial structures of personality ratings from different sources. Journal of Abnormal and Social Psychology, 44, 329-344.

Flores, E., Ciccetti, D., \& Rogosch, F.A., (2005). Predictors of resilience in maltreated and nonmaltreated Latino children. Developmental Psychology, 41, 338-351.

Fraley, R. C., \& Roberts, B. W. (2005). Patterns of continuity: A dynamic model for conceptualizing the stability of individual differences in psychological constructs across the life course. Psychological Review, 112, 60-74.

Funder, D., \& Block, J. (1989). The role of ego-control, ego-resiliency, and IQ in delay of gratification in adolescence. Journal of Personality and Social Psychology, 57, 10411050.

Gaughan, E.T., Miller, J.D., \& Lynam, D.R. (2012). Examining the utility of general models of personality in the study of psychopathy: A comparison of the HEXACO-PI-R and the NEO-PI-R. Journal of Personality Disorders, 26, 513-523.

Goldberg. L. R. (1981). Language and individual differences: The search for universals in personality lexicons. In L. Wheeler (Ed.), Review of Personality and Social Psychology (Vol. 2, pp. 141-165). Beverly Bilis. CA: Sage.

Gramzow, R. H., Sedikides, C., \& Panter, A. T. (2004). Patterns of self-regulation and the Big Five. European Journal of Personality, 18, 367-385.

Grumm, M., \& von Collani, G. (2009). Personality types and self-reported aggressiveness. Personality and Individual Differences, 47, 845-850.

Hahn, D. W., Lee, K., \& Ashton, M. C. (1999). A factor analysis of the most frequently used Korean personality trait adjectives. European Journal of Personality, 13, 261-282.

Harré, R. (1998). The singular self: An introduction to the psychology of personhood. London: Sage. 
Hart, D., Atkins, R., \& Fegley, S. (2003). Personality and development in childhood: A personcentred approach. Monographs of the Society for Research in Child Development, 68, 1-109.

Hart, D., Hofmann, V., Edelstein, W., \& Keller, M. (1997). The relation of childhood personality types to adolescent behavior and development: A longitudinal study of Icelandic children. Developmental Psychology, 33, 195-205.

Herzberg, P.Y., \& Roth, M. (2006). Beyond resilients, undercontrollers, and overcontrollers? An extension of personality prototypes research. European Journal of Personality, 20, $5-28$

Howarth, E., \& Browne, J. A. (1971). An item-factor-analysis of the 16 PF, Personality: An International Journal, 2, 119-139.

Huang, D. (N.D.). CALDAR UCLA: Center for Advancing Longitudinal Drug Abuse Research: Frequently asked questions. Retrieved from http://www.caldar.org/html/faq.html.

Huey, S.J., Jr, \& Weisz, J.R. (1997). Ego control, ego resiliency, and the five-factor model as predictors of behavioral and emotional problems in clinic-referred children and adolescents. Journal of Abnormal Psychology, 106, 404- 415.

Isler, L., Fletcher, G.J.O., Liu, J.H, \& Sibley, C.G., (2017). Validation of the four-profile configuration of personality types within the Five-Factor Model. Personality and Individual Differences, 106, 257-262

Isler, L., Liu, J.H., Sibley, C.G., \& Fletcher, G.J.O. (2016). Self-regulation and personality profiles: Empirical development, longitudinal stability and predictive ability. European Journal of Personality, 30, 274-287

John, O. P. (1990). The "Big Five" factor taxonomy: Dimensions of personality in the natural language and questionnaires. In L. A. Pervin (Ed.) Handbook of personality: Theory and research (pp. 66-100) New York: Guilford Press.

John, O. P., \& Srivastava, S. (1999). The big five trait taxonomy: History, measurement, and theoretical perspectives. In L. A. Pervin \& O. P. John (Eds.), Handbook of personality: Theory and research (2nd ed., pp. 102-138). New York: Guilford Press. 
Kobasa, S. C. (1979). Stressful life events, personality, and distress: An inquiry into hardiness. Journal of Personality and Social Psychology, 37, 1-11.

Kremen, A.M., \& Block, J.H. (1998). The roots of ego-control in young adulthood: Links with parenting in early childhood. Journal of Personality and Social Psychology, 75, 10621075.

Lamiell, J.T. (1997). Individuals and the differences between them. In Handbook of Personality Psychology, ed. R Hogan, J Johnson, S Briggs, pp. 117-141. San Diego: Academic.

Lee, K., \& Ashton, M. C. (2008). The HEXACO personality factors in the indigenous personality lexicons of English and 11 other languages. Journal of Personality, 76, 1001-1053.

Lee, K., Ashton, M. C., \& de Vries, R. (2005). Predicting workplace delinquency and integrity with the HEXACO and five-factor models of personality structures. Human Performance, 18, 179-197.

Lee, K., Ashton, M. C., Pozzebon, J.A., Visser, B.A., Bourdage, J.S. \& Ogunfowora, B. (2009). Similarity and assumed similarity in personality reports of well-acquainted persons. European Journal of Personality, 27, 169-184.

Legault, L., Green-Demers, I., Grant, P., \& Chung, J. (2007). On the self-regulation of implicit and explicit prejudice: A self-determination perspective. Personality and Social Psychology Bulletin, 33, 732-749.

Letzring, T. D., Block, J., \& Funder, D. C. (2005). Ego-control and ego-resiliency: Generalization of self-report scales based on personality descriptions from acquaintances, clinicians, and the self. Journal of Research in Personality, 39, 395422.

Licht, A.N. (2007). The entrepreneurial spirit and what the law can do about it. Comparative Labor Law \& Policy Journal, 28, 817-861.

Little, R.J.A., \& Rubin, D.B. (2002). Statistical Analysis with Missing Data, 2nd ed. Wiley, New York. 
Norman, W. T. (1963). Toward an adequate taxonomy of personality attributes: Replicated factor structure in peer nomination personality ratings. Journal of Abnormal and Social Psychology, 66, 574-583.

Marsh, H. W., Lüdtke, O., Trautwein, U., \& Morin, A. J. S. (2009). Classical latent profile analysis of academic self-concept dimensions: Synergy of person- and variablecentered approaches to theoretical models of self-concept. Structural Equation Modeling: A Multidisciplinary Journal, 16, 191-225.

McCrae, R.R. (1989). 'Why I advocate the Five-Factor Model: Joint analyses of the NEO-PI and other instruments', in D.M. Buss and N.Cantor (eds), Personality Psychology: Recent Trends and Emerging Directions. New York: Springer-Verlag, pp. 237-245

McCrae, R. R., \& Allik, J. (Eds.). (2002). The Five-Factor Model of personality across cultures. New York: Kluwer Academic/Plenum Publishers.

McCrae, R.R., \& Costa, P.T., Jr. (1985). Updating Norman's "adequate taxonomy": Intelligence and personality dimensions in natural language and in questionnaires. Journal of Personality and Social Psychology, 49, 710-721.

McCrae, R.R., \& Costa, P.T., Jr. (1989). Reinterpreting the Myers-Briggs Type Indicator from the perspective of the Five-Factor Model of personality. Journal of Personality, 57, $17-40$.

McCrae, R.R., \& Costa, P.T., Jr. (1997). Personality trait structure as a human universal. American Psychologist, 52, 509-516.

McCrae, R.R., \& Costa, P.T., Jr. (2008). Empirical and theoretical status of the Five-Factor Model of personality traits. In G. Boyle, G. Matthews, \& D. Saklofske (Eds.), Sage handbook of personality theory and assessment (Vol. 1, pp. 273-294). Los-Angeles, CA: Sage Publications.

McCrae, R. R., \& John, O. P. (1992). An introduction to the five-factor model and its applications. Journal of Personality, 60, 175-215. 
McCrae, R. R., Terracciano, A., \& 78 Members of the Personality Profiles of Cultures Project. (2005a). Universal features of personality traits from the observer's perspective: Data from 50 cultures. Journal of Personality and Social Psychology, 88, 547-561.

McLachlan G.J., \& Peel D. (2000). Finite mixture models. New York: Wiley.

Meeus, W., Van de Schoot, R., Klimstra, T., \& Branje, S. (2011). Personality types in adolescence: Change and stability and links with adjustment and relationships: A five-wave longitudinal study. Developmental Psychology, 47(4), 1181-1195.

Messick, S. (1995). Validity of psychological assessment: Validation of inferences from persons' responses and performances as scientific inquiry into score meaning. American Psychologist, 50, 741-749

Muthén L., \& Muthén B. (2012). MPLUS (Version 7.1) [Statistical software]. Los Angeles, CA.

Nagin D.S. (2005). Group-based Modeling of Development. Cambridge, MA: Harvard University Press.

Nagin D. S. \& Tremblay R.E. (2005). Developmental trajectory groups: fact or a useful statistical fiction? Criminology, 4, 873-904.

No, S., Hong, Y-Y., Liao, H-Y., Lee, K., Wood, D., \& Chao, M. M. (2008). Lay theory of race affects and moderated Asian Americans' responses toward American culture. Journal of Personality and Social Psychology, 95, 991-1004.

Nylund, K.L., Asparouhov, T, \& Muthén, B.O. (2007). Deciding on the number of classes in a Latent Class Analysis and Growth Mixture Modeling: A Monte Carlo simulation study. Structural Equation Modeling, 14, 535-569.

Ozer, D.J., \& Benet-Martinez, V. (2006). Personality and the prediction of consequential outcomes. Annual Review of Psychology, 57, 401-421.

Paunonen, S. V. (2002). Design and construction of the Supernumerary Personality Inventory (Research Bulletin 763). London, Ontario: University of Western Ontario.

Paunonen, S.V., \& Ashton, M.C. (2001). Big Five factors and facets and the prediction of behaviour. Journal of Personality and Social Psychology, 81, 524-539. 
Piedmont, R.L., \& Chae, J.H. (1997). Cross-cultural generalizability of the Five-Factor Model of personality: Development and validation of the NEO PI-R for Koreans. Journal of Cross-Cultural Psychology, 28, 131-155

Raftery, A. E. (1995). "Bayesian Model Selection in Social Research (With Discussion)." Pp. 111-63 in Sociological Methodology 1995, edited by P. V. Marsden. Cambridge, MA: Blackwell.

Rammstedt, B., Riemann, R., Angleitner, A., \& Borkenau, P. (2004). Resilients, overcontrollers, and undercontrollers: The replicability of the three personality prototypes across informants. European Journal of Personality, 18, 1-14.

Roberts, B. W., \& DelVecchio, W. F. (2000). The rank-order consistency of personality traits from childhood to old age: A quantitative review of longitudinal studies. Psychological Bulletin, 126, 3-25

Roberts, B.W., Kuncel, N.R., Shiner, R., Caspi, A. \& Goldberg, L.R. (2007). The power of personality: The comparative validity of personality traits, socioeconomic status, and cognitive ability for predicting important life outcomes. Perspectives on Psychological Science, 2, 313-345.

Roberts, B.W., Walton, K.E., \& Viechtbauer, W. (2006). Patterns of mean-level change in personality traits across the life course: A meta-analysis of longitudinal studies. Psychological Bulletin, 132, 1-25.

Robins, R. W., John, O. P., Caspi, A., Moffitt, T. E., \& Stouthamer-Loeber, M. (1996). Resilient, overcontrolled, and undercontrolled boys: Three replicable personality types. Journal of Personality and Social Psychology, 7, 157-171.

Robins, R. W., \& Tracy, J. L. (2003). Setting an agenda for a person-centered approach to personality development. Monographs of the Society for Research in Child Development, 68, 110-122.

Rosenberg, M. (1965). Society and the Adolescent Self-image. Princeton, NJ: Princeton University Press. 
Roth, M., \& von Collani, G. (2007). A head-to-head comparison of big-five types and traits in the prediction of social attitudes: Further evidence for a five-cluster typology. Journal of Individual Differences, 28, 138-149.

Sarracino, D., Presaghi, F., Degni, S, \& Innamorati, M. (2011). Sex-specific relationships among attachment security, social values, and sensation seeking in early adolescence: Implications for adolescents' externalizing problem behaviour. Journal of Adolescence, 34, 541-554.

Saucier, G. (2009). Recurrent personality dimensions in inclusive lexical studies: Indications for a Big Six structure. Journal of Personality, 77, 1577-1614

Saucier, G., \& Goldberg, L.R. (2001). Lexical studies of indigenous personality factors: Premises, products, and prospects. Journal of Personality, 69, 847-879.

Schwartz, S. H. (1992). Universals in the content and structure of values: Theoretical advances and empirical tests in 20 countries. Advances in Experimental Social Psychology, 25, 1-65.

Schwarz, G. (1978). Estimating the dimension of a model. Annals of Statistics 6, 461-464.

Schwartz, S. H. (2011). Studying values: Personal adventure, future directions. Journal of Cross-Cultural Psychology, 42, 307-319.

Sell, S. B., Demaree, B. G., \& Will, D. P. (1970). Dimensions of personality. I. Conjoint factor structure of Guilford and Cattell trait markers. Multivariate Behavioral Research, 5, $391-422$

Shelton, J. N., \& Richeson, J. A. (2005). Intergroup Contact and Pluralistic Ignorance. Journal of Personality and Social Psychology, 88, 91-107.

Sibley, C. G., (2009). Archive of NZAVS questionnaires. NZAVS Technical Documents, e06. Available at: www.psych.auckland.ac.nz/uoa/NZAVS

Sibley, C. G., Harding, J. F., Perry, R., Asbrock, F., \& Duckitt, J. (2010). Personality and prejudice: Extension to the HEXACO personality model. European Journal of Personality, 24, 515-534. 
Sibley, C. G., Luyten, N., Purnomo, M., Moberly, A., Wootton, L. W., Hammond, M. D., Sengupta, N., Perry, R., Hoverd, W. J., \& Robertson, A. (2011). The Mini-IPIP6: Validation and extension of a short measure of the Big-Six factors of personality in New Zealand. New Zealand Journal of Psychology, 40, 142-159.

Sidanius, J., \& Pratto, F. (1999). Social dominance: An intergroup theory of social hierarchy and oppression. Cambridge: Cambridge University Press.

Steca P., Alessandri, G., \& Caprara, G.V. (2010). The utility of a well-known personality typology in studying successful aging: Resilients, undercontrollers, and overcontrollers in old age. Personality and Individual Differences, 4(48), 442-446.

Stephan, W. G., \& Stephan, C. W. (1985). Intergroup anxiety. Journal of Social Issues, 41, 157-175.

Stern, P. C., Guagnano, G. A., \& Dietz, T. (1998). A brief inventory of values. Educational and Psychological Measurement, 58, 984-1001.

Szirmak, Z., \& De Raad, B. (1994). Taxonomy and structure of Hungarian personality traits. European Journal of Personality, 8, 95-117.

Tein, J.Y., Coxe, S., \& Cham, H. (2013). Statistical power to detect the correct number of classes in latent profile analysis. Structural Equation Modelling, 20, 640-657.

Tupes. E. C., \& Christal. R. E. (1961). Recurrent personality factors based on trait ratings (USAF ASD Tech. Rep. No. 61-97). Lackland Air Force Base. TX: U.S. Air Force

Van Kampen, D. (2012). The 5-Dimensional Personality Test (5DPT): Relationships with two lexically based instruments and validation of the Absorption Scale. Journal of Personality Assessment, 94, 92-101.

Weller, J. A., \& Thulin, E. W. (2012). Do honest people take fewer risks? Personality correlates of risk-taking to achieve gains and avoid losses in HEXACO space. Personality and Individual Differences, 53, 923-926.

Weller, J. A., \& Tikir, A. (2011). Predicting domain-specific risk taking with the HEXACO personality structure. Jounral of Behavioral Decision Making, 24, 180-201. 


\section{Appendix A: Personality Scale Factor Loadings and Descriptive Statistics}

Six Factor Model of Personality: Factor loadings and descriptive statistics (Study 1)

\begin{tabular}{|c|c|c|c|c|c|c|c|c|}
\hline & $\mathrm{H}$ & $\mathrm{E}$ & 0 & ES & A & C & $M$ & $S D$ \\
\hline Deserve more things in life $(r)$ & .79 & & & & & & 4.84 & 1.75 \\
\hline $\begin{array}{l}\text { Would like to be seen driving around in a very } \\
\text { expensive car ( } r \text { ) }\end{array}$ & .77 & & & & & & 5.18 & 1.88 \\
\hline $\begin{array}{l}\text { Would get a lot of pleasure from owning } \\
\text { expensive luxury goods ( } r \text { ) }\end{array}$ & .75 & & & & & & 4.59 & 1.87 \\
\hline Feel more entitled to more of everything $(r)$ & 71 & & & & & & 5.53 & 1.47 \\
\hline Keep in the background $(r)$ & & .75 & & & & & 4.02 & 1.52 \\
\hline Am the life of the party & & .74 & & & & & 3.60 & 1.45 \\
\hline Don't talk a lot (r) & & .72 & & & & & 4.36 & 1.63 \\
\hline Talk to a lot of different people at parties & & .71 & & & & & 4.20 & 1.73 \\
\hline Have difficulty understanding abstract ideas (r) & & & .75 & & & & 4.60 & 1.58 \\
\hline Am not interested in abstract ideas $(r)$ & & & .75 & & & & 4.75 & 1.56 \\
\hline Do not have a good imagination (r) & & & .69 & & & & 5.15 & 1.62 \\
\hline Have a vivid imagination & & & .51 & & & & 4.57 & 1.58 \\
\hline Have frequent mood swings ( $r$ ) & & & & .74 & & & 3.17 & 1.66 \\
\hline Am relaxed most of the time & & & & .71 & & & 3.24 & 1.41 \\
\hline Get upset easily $(r)$ & & & & .70 & & & 3.56 & 1.58 \\
\hline Seldom feel blue & & & & .61 & & & 3.80 & 1.63 \\
\hline Sympathise with others' feelings & & & & & .82 & & 5.55 & 1.19 \\
\hline Feel others' emotions & & & & & .80 & & 5.23 & 1.34 \\
\hline Am not really interested in others $(r)$ & & & & & .57 & & 5.31 & 1.45 \\
\hline Am not interested in other people's problems $(r)$ & & & & & .52 & & 4.87 & 1.61 \\
\hline $\begin{array}{l}\text { Often forget to put things back in their proper } \\
\text { place }(r)\end{array}$ & & & & & & .72 & 4.98 & 1.78 \\
\hline Get chores done right away & & & & & & .72 & 4.75 & 1.52 \\
\hline Like order & & & & & & .69 & 5.35 & 1.44 \\
\hline Make a mess of things ( $r$ ) & & & & & & .66 & 5.32 & 1.40 \\
\hline
\end{tabular}

Note. Maximum likelihood estimation and orthogonal (varimax) rotation were applied. Cross-loadings smaller than .3 are supressed.

A, Agreeableness; C, Conscientiousness; E, Extraversion; ES, Emotional Stability; H, Honesty-humility; O, Openness. Negatively worded items $(r)$ were reverse coded 
Five Factor Model of Personality: Factor loadings and descriptive statistics (Study 2)

\begin{tabular}{|c|c|c|c|c|c|c|c|}
\hline & $\mathrm{E}$ & $\mathrm{O}$ & ES & A & C & $M$ & $S D$ \\
\hline Am the life of the party & .80 & & & & & 3.60 & 1.45 \\
\hline Talk to a lot of different people at parties & .75 & & & & & 4.20 & 1.73 \\
\hline Keep in the background $(r)$ & .69 & & & & & 4.02 & 1.52 \\
\hline Don't talk a lot $(r)$ & .65 & & & & & 4.36 & 1.63 \\
\hline Have difficulty understanding abstract ideas ( $r$ ) & & .75 & & & & 4.60 & 1.58 \\
\hline Am not interested in abstract ideas $(r)$ & & .74 & & & & 4.75 & 1.56 \\
\hline Do not have a good imagination (r) & & .68 & & & & 5.15 & 1.62 \\
\hline Have a vivid imagination & & .46 & .32 & & & 4.57 & 1.58 \\
\hline Have frequent mood swings ( $r$ ) & & & .78 & & & 3.17 & 1.66 \\
\hline Get upset easily ( $r$ ) & & & .72 & & & 3.56 & 1.58 \\
\hline Am relaxed most of the time & & & .65 & & & 3.24 & 1.41 \\
\hline Seldom feel blue & & & .58 & & & 3.80 & 1.63 \\
\hline Sympathise with others' feelings & & & & .79 & & 5.55 & 1.19 \\
\hline Feel others' emotions & & & & .76 & & 5.23 & 1.34 \\
\hline Am not really interested in others $(r)$ & & & & .64 & & 5.31 & 1.45 \\
\hline Am not interested in other people's problems ( $r$ ) & & & & .59 & & 4.87 & 1.61 \\
\hline Get chores done right away & & & & & .73 & 4.75 & 1.52 \\
\hline $\begin{array}{l}\text { Often forget to put things back in their proper place } \\
\text { (r) }\end{array}$ & & & & & .72 & 4.98 & 1.78 \\
\hline Like order & & & & & .69 & 5.35 & 1.44 \\
\hline Make a mess of things $(r)$ & & & & & .65 & 5.32 & 1.40 \\
\hline
\end{tabular}

Note. Maximum likelihood estimation and orthogonal (varimax) rotation were applied. Cross-loadings smaller than .3 are supressed.

A, Agreeableness; C, Conscientiousness; E, Extraversion; ES, Emotional Stability; H, Honesty-humility; O, Openness. Negatively worded items ( $r$ ) were reverse coded 
Six Factor Model of Personality: Factor loadings and descriptive statistics (Study 3)

\begin{tabular}{|c|c|c|c|c|c|c|c|c|}
\hline & $\mathrm{E}$ & ES & A & $\mathrm{H}$ & $\mathrm{O}$ & C & $M$ & $S D$ \\
\hline Keep in the background $(r)$ & .82 & & & & & & 4.21 & 1.58 \\
\hline Talk to a lot of different people at parties & .80 & & & & & & 3.93 & 1.80 \\
\hline Am the life of the party & .78 & & & & & & 3.11 & 1.57 \\
\hline Don't talk a lot $(r)$ & .75 & & & & & & 4.58 & 1.78 \\
\hline Have frequent mood swings ( $r$ ) & & .86 & & & & & 5.07 & 1.56 \\
\hline Seldom feel blue & & .74 & & & & & 4.22 & 1.82 \\
\hline Get upset easily ( $r$ ) & & .73 & & & & & 4.49 & 1.65 \\
\hline Am relaxed most of the time & & .72 & & & & & 4.75 & 1.52 \\
\hline Make a mess of things $(r)$ & & .50 & & & & .43 & 5.35 & 1.36 \\
\hline Sympathize with others' feelings & & & .85 & & & & 5.55 & 1.28 \\
\hline Feel others' emotions & & & .79 & & & & 5.27 & 1.34 \\
\hline Am not interested in other people's problems ( $r$ ) & & & .75 & & & & 5.33 & 1.47 \\
\hline Am not really interested in others $(r)$ & .37 & & .66 & & & & 5.56 & 1.59 \\
\hline Deserve more things in life $(r)$ & & & & .78 & & & 4.76 & 1.58 \\
\hline Feel entitled to more of everything ( $r$ ) & & & & .76 & & & 5.19 & 1.58 \\
\hline $\begin{array}{l}\text { Would like to be seen driving around in a very } \\
\text { expensive car }(r)\end{array}$ & & & & .76 & & & 5.08 & 1.85 \\
\hline $\begin{array}{l}\text { Would get a lot of pleasure from owning expensive } \\
\text { luxury goods }(r)\end{array}$ & & & & .73 & & & 4.13 & 1.80 \\
\hline Have difficulty understanding abstract ideas (r) & & & & & .81 & & 5.40 & 1.52 \\
\hline Am not interested in abstract ideas $(r)$ & & & & & .81 & & 5.33 & 1.62 \\
\hline Have a vivid imagination & & & & & .65 & & 4.96 & 1.58 \\
\hline Do not have a good imagination $(r)$ & & & & & .65 & & 5.35 & 1.66 \\
\hline $\begin{array}{l}\text { Often forget to put things back in their proper } \\
\text { place }(r)\end{array}$ & & & & & & .80 & 4.86 & 1.77 \\
\hline Like order & & & & & & .72 & 5.40 & 1.25 \\
\hline Get chores done right away & & & & & & .67 & 4.23 & 1.65 \\
\hline
\end{tabular}

Note. Maximum likelihood estimation and orthogonal (varimax) rotation were applied. Cross-loadings smaller than .3 are supressed.

A, Agreeableness; C, Conscientiousness; E, Extraversion; ES, Emotional Stability; H, Honesty-humility; O, Openness. Negatively worded items ( $r$ ) were reverse coded 


\section{Appendix B: Scale Items and Instructions ${ }^{6}$}

\section{Personality:}

Personality items were administered with the following instructions: "This part of the questionnaire measures your personality. Please circle the number that best represents how accurately each statement describes you." Items were rated on a scale ranging from 1 (very inaccurate) to 7 (very accurate).

\begin{tabular}{|c|c|}
\hline \multicolumn{2}{|c|}{ Extraversion } \\
\hline- & Keep in the background $(r)$ \\
\hline- & Talk to a lot of different people at parties \\
\hline- & Am the life of the party \\
\hline- & Don't talk a lot $(r)$ \\
\hline \multicolumn{2}{|c|}{ Emotional Stability } \\
\hline- & Have frequent mood swings $(r)$ \\
\hline- & Seldom feel blue \\
\hline- & Get upset easily $(r)$ \\
\hline- & Am relaxed most of the time \\
\hline \multicolumn{2}{|c|}{ Agreeableness } \\
\hline- & Am not really interested in others ( $r$ ) \\
\hline- & Sympathize with others' feelings \\
\hline- & Feel others' emotions \\
\hline- & Am not interested in other people's problems $(r)$ \\
\hline \multicolumn{2}{|c|}{ Conscientiousness } \\
\hline- & Make a mess of things $(r)$ \\
\hline- & Often forget to put things back in their proper place $(r)$ \\
\hline- & Like order \\
\hline- & Get chores done right away \\
\hline \multicolumn{2}{|c|}{ Openness } \\
\hline- & Am not interested in abstract ideas ( $r$ ) \\
\hline- & Have a vivid imagination \\
\hline- & Do not have a good imagination $(r)$ \\
\hline- & Often forget to put things back in their proper place $(r)$ \\
\hline \multicolumn{2}{|r|}{ Honesty-Humility } \\
\hline- & Deserve more things in life $(r)$ \\
\hline- & Feel entitled to more of everything $(r)$ \\
\hline- & Would like to be seen driving around in a very expensive car (r) \\
\hline- & Would get a lot of pleasure from owning expensive luxury goods ( $r$ ) \\
\hline
\end{tabular}

\footnotetext{
${ }^{6}$ Negatively worded items $(r)$ were reverse coded
} 


\section{Subjective wellbeing}

Self-Esteem items were administered with the following instructions: "This part of the questionnaire measures your personality. Please circle the number that best represents how accurately each statement describes you." Items were rated on a scale ranging from 1 (very inaccurate) to 7 (very accurate).

\section{Self-Esteem}

- On the whole am satisfied with myself

- Take a positive attitude toward myself

- Am inclined to feel that I am a failure (r)

Personal Wellbeing items were administered with the following instructions: "The following items assess your current satisfaction with different aspects of your life and aspects of New Zealand more generally. Please rate how satisfied you are with each aspect of your life and/or New Zealand society using the scale below." Items were rated on a scale ranging from 1 (completely dissatisfied) to 10 (completely satisfied).

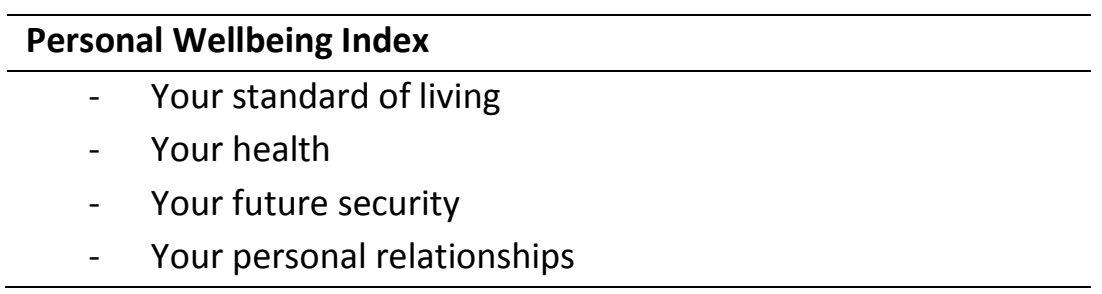

Satisfaction with Life items were administered with the following instructions: "The statements below reflect different opinions and points of view. Please indicate how strongly you disagree or agree with each statement. Remember, the best answer is your own opinion" and were rated on a scale ranging from 1 (strongly disagree) to 7 (strongly agree).

\begin{tabular}{cl}
\hline Satisfaction with Life \\
\hline$-\quad$ I am satisfied with my Life \\
$-\quad$ In most ways my life is close to ideal \\
\hline
\end{tabular}




\section{Perceived Quality of Interethnic Relations}

All items measuring perceived quality of interethnic relations were administered with the following instructions: "The statements below reflect different opinions and points of view. Please indicate how strongly you disagree or agree with each statement. Remember, the best answer is your own opinion" and were rated on a scale ranging from 1 (strongly disagree) to 7 (strongly agree).

\section{Symbolic Threat Perception}

- $\quad$ NZ Europeans have different values and morals compared to most other NZers

- Maori have different values and morals compared to most other NZers

- Pacific Islanders have different values and morals compared to most other NZers

- Asians have different values and morals compared to most other NZers

\section{Realistic Threat Perception}

- In my opinion, more good jobs for NZ Europeans means fewer good jobs for members of other groups in New Zealand

- In my opinion, more good jobs for Maori means fewer good jobs for members of other groups in New Zealand

- In my opinion, more good jobs for Pacific Islanders means fewer good jobs for members of other groups in New Zealand

- In my opinion, more good jobs for Asians means fewer good jobs for members of other groups in New Zealand

Racial Essentialism

- To a large extent, a person's race biologically determines his or her abilities

Intergroup Anxiety

- I feel anxious about interacting with people from other races

\section{Race-based Rejection Sensitivity}

- People from other races would be likely to reject my on the basis of my race 


\section{Social Dominance Orientation}

Items measuring Social Dominance Orientation were administered with the following instructions: "The statements below reflect different opinions and points of view. Please indicate how strongly you disagree or agree with each statement. Remember, the best answer is your own opinion" and were rated on a scale ranging from 1 (strongly disagree) to 7 (strongly agree).

\section{Social Dominance Orientation}

- It is OK if some groups have more of a chance in life than others

- Inferior groups should stay in their place

- To get ahead in life, it is sometimes okay to step on other groups

- We should have increased social equality

- It would be good if groups could be equal

- We should do what we can to equalise conditions for different groups

\section{Self-Enhancement and Openness to Change Values}

Items measuring Self-Enhancement and Openness to Change values were administered with the following instructions: "Please circle the number that best represents how important each of the following values is for you as a guiding principle in your life. Use the scale below to rate these items." Items were rated on a scale which ranged from -1 (opposed to my values) to 0 (not important) to 3 (important) to 6 (very important) to 7 (of supreme importance).

\section{Self-Enhancement}

- AUTHORITY (the right to lead or command)

- INFLUENCE (having an impact on people and events)

- WEALTH (material possessions, money)

\begin{tabular}{cc}
\hline Openness to Change \\
\hline - & A VARIED LIFE (filled with challenged, novelty and change) \\
- & AN EXCITING LIFE (stimulating experiences) \\
- & CURIOSITY (interest in everything, exploring) \\
\hline
\end{tabular}




\section{Ego-Resiliency and Ego-Control}

Items measuring Ego-Resiliency and Ego-Control were administered with the following instructions: "This questionnaire measures your feelings about yourself and your experiences. Please circle the number that best represents how accurately each statement describes you now, not as you wish to be in the future." Items were rated on a scale which ranged from 1 (Strongly Disagree) to 4 (Strongly Agree).

\section{Ego-Resiliency}

- I am generous with my friends

- I quickly get over and recover from being startled

- I enjoy dealing with new and unusual situations

- I usually succeed in making a favourable impression on people

- I enjoy trying new foods I have never tasted before

- I am regarded as a very energetic person

- I like to take different paths to familiar places

- I am more curious than most people

- Most of the people I meet are likeable

- I usually think carefully about something before acting

- I like to do new and different things

- My daily life is full of things that keep me interested

- I would be willing to describe myself as pretty 'strong' personality

- I get over my anger at someone reasonably quickly

Ego-Control

- $\quad$ I tend to buy things on impulse $(r)$

- I become impatient when I have to wait for something (r)

- I often say and do things on the spur of the moment, without stopping to think ( $r$ )

- I can remember 'playing sick' to get out of something (r)

- I have often had to take orders from someone who did not know as much as I did (r)

- When I get bored, I like to stir up some excitement (r)

- Some of my family have quick tempers ( $r$ )

- People consider me a spontaneous, devil-may-care person ( $r$ )

- I often get involved in things I later wish I could get out of ( $r$ )

- I have been known to do unusual things on a dare ( $r$ )

- I have sometimes stayed away from another person because I thought I might do or say something that I might regret otherwise $(r)$

- I do not always tell the truth (r)

- My way of doing things can be misunderstood or bother others ( $r$ )

- Sometimes I rather enjoy going against the rules and doing things I am not supposed to do (r)

- At times, I am tempted to do or say something that others would think inappropriate (r)

- At times I have very much wanted to leave home ( $r$ )

- I would like to be a journalist (r) 
- I like to flirt (r)

- Some of my family have habits that bother and annoy me very much ( $r$ )

- At times I have worn myself out by undertaking too much ( $r$ )

- In a group of people I would not be embarrassed to be called on to start a discussion or give an opinion about something I know well $(r)$

- I would like to wear expensive clothes ( $r$ )

- I am against giving money to homeless people

- It is unusual for me to express strong approval or disapproval of the actions of others

- I like to stop and think things over before I do them

- I don't like to start a new project until I know exactly how to proceed

- I finish one activity or project before starting another

- I am steady and planful rather than unpredictable and impulsive

- On the whole, I am a cautious person

- I do not let too many things get in the way of work

- I keep out of trouble at all costs

- I consider a matter from every viewpoint before I make a decision

- I am easily downed in an argument

- I have never done anything dangerous for the fun of it

- My conduct is largely controlled by the customs of those about me

- It makes me uncomfortable to put on a stunt at a party even when others are doing the same thing

- I find it hard to make small talk when I meet new people 